\title{
Vascular Cognitive Impairment \\ Phenomenology, Course, Risk Factors
}




$\begin{array}{ll}\text { Cover } & \text { 'Het Brein Beroert' SMC Rasquin } \\ \text { Vormgeving } & \text { RenéRutten.nl } \\ \text { Production } & \text { Ponsen \& Looijen, Wageningen } \\ \text { ISBN } & 90-75579-21-7\end{array}$

(c) S.M.C. Rasquin, 2004, Maastricht, The Netherlands

Neuropsych Publishers is a nonprofit organization, which aims at promoting the science of 'Brain and Behavior' and improving the application of the products of this science in health care and education. Neuropsych Publishers accomplishes these aims by publishing books, dissertations, and other products of scientific activity, by disseminating educational materials and publication of tests, assessment scales and other psychometric instruments in the field of Neuropsychology, Neuropsychiatry, and other arrears within the domain of Brain and Behavior.

Neuropsych Publishers

Department of Psychiatry and Neuropsychology

Maastricht University

P.O. Box 616

NL-6200 MD Maastricht

The Netherlands

www-np.unimaas.nl 


\title{
Vascular Cognitive Impairment
}

\author{
Phenomenology, Course, Risk Factors
}

PROEFSCHRIFT

ter verkrijging van de graad van doctor aan de Universiteit Maastricht op gezag van Rector Magnificus,

Prof. mr. G.P.M.F. Mols volgens het besluit van het College van Decanen, in het openbaar te verdedigen op vrijdag 3 december 2004 om 14.00 uur.

door

\section{Sascha Maria Cornelia Rasquin}

Geboren te Maastricht op 20 november 1976. 


\section{Promotores}

Prof. dr. F.R.J. Verhey

Em. Prof. dr. J. Troost

\section{Beoordelingscommissie}

Prof. dr. J. Jolles (voorziter)

Prof. dr. T. J. Heeren (Universiteit Utrecht)

Dr. C.M. van Heugten

Prof. dr. M. Limburg

Prof. dr. Ph. Scheltens (Vrije Universiteit Amsterdam)

The research described in this thesis was performed in the Maastricht Brain \& Behavior Institute, and the department of Psychiatry \& Neuropsychology, Maastricht University, Maastricht, the Netherlands, in collaboration with the department Neurology, Academic Hospital Maastricht

The CODAS study that is described in this thesis was supported by grants of the Adriana van Rinsum-Ponsen Foundation.

Financial support by the Netherlands Heart Foundation for the publication of this thesis is gratefully acknowledged.

The publication of this thesis was financially supported by:

Boehringer Ingelheim, Viatris, Het Remmert Adriaan Laan Foundation, UCB Pharma, van Leersum KNAW Foundation, Sigma Tau Ethifarm, Lundbeck, Servier Nederland, Internationale Stichting Alzheimer Onderzoek, Ab Laane Foundation, Adriana van Rinsum-Ponsen Foundation, iRv: Kenniscentrum voor Revalidatie en Handicap. 
Wijsheid verwerven is zoveel beter dan goud,

Inzicht verwerven is te verkiezen boven zilver

Spreuken; 16,16

Willibrodvertaling

Voor mijn ouders 



\section{Contents}

I General Introduction 9

II Vascular Cognitive Impairment: a new concept 15

III Methodological considerations of CODAS 23

IV Vascular cognitive disorders: memory, mental speed, 35 and cognitive flexibility after stroke

V Cognitive functioning after stroke: a one year follow-up study $\quad 45$

VI The effect of different diagnostic criteria on the prevalence 59 and incidence of post-stroke dementia

VII Predictive accuracy of Mild Cognitive Impairment subtypes for $\quad 75$ Alzheimer's disease and vascular dementia

VIII Predictors of reversible Mild Cognitive Impairment 89

IX Demographic and CT scan features related to cognitive 101 impairment

X Progressive brain damage in relation to cognitive performance 119 after stroke

XI Checklist for cognitive and emotional consequences following 133 stroke (CLCE-24): development and initial validation

XII The influence of psychiatric symptoms on cognitive 147 performance after stroke

XIII General discussion

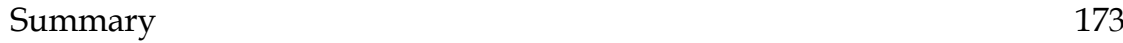

$\begin{array}{lr}\text { Samenvatting } & 179\end{array}$

$\begin{array}{ll}\text { References } & 185\end{array}$

$\begin{array}{ll}\text { Publications } & 203\end{array}$

$\begin{array}{ll}\text { Dankwoord } & 207\end{array}$

$\begin{array}{ll}\text { Curriculum vitea } & 213\end{array}$ 
General Introduction 


\section{Case history}

A 68-year old retired notary was referred by his general practitioner to the outpatient stroke unit because the left corner of his mouth was a little bit wry, his right arm and leg were paralyzed, and he slurred his words. He was admitted to the stroke unit for lacunar infarction in the left hemisphere, and was treated with an anti-coagulant, and speech and physical therapy were started. Within 2 days his speech had returned to normal and so the speech therapy was stopped. However, he did not regain strength in his limbs, and after 8 days he was admitted to the rehabilitation department of a nursing home. Daily physical therapy was planned. After a week the doctor assessed whether the patient had regained strength in his limb and asked about his memory function. The patient felt that he had progressed with physical therapy and that there was nothing wrong with his memory -he still recognised his grandchildren. The patient had an MMSE score within the normal range (27). After 3 months his arm and leg had regained almost normal strength and the patient was discharged home. Here, the patient started to notice that he had some problems performing daily activities. Although he could walk again, he had problems with his hobby as secretary of the local choir. At meetings he had difficulty following what was said, and by whom and thus he could not make proper notes. He complained that everything went too fast and that he could not keep up with conversations or with the subtitles in movies. He also felt insecure and depressed.

Several questions arise from this case history:

Is this cognitive profile typical for patients with a first-ever stroke?

What is the relation between brain damage due to stroke and cognitive performance?

What does this patient's feeling of depression have to do with stroke?

\section{Introduction}

Stroke is, after myocardial infarction and cancer, the third leading cause of death in Western countries and is associated with a high level of permanent handicap in the elderly. In the Netherlands, 30000 patients are diagnosed annually as having had a first-ever cerebral stroke, and of these $25 \%$ die within 1 month. Annually, over 3\% of the Dutch healthcare budget is spent on strokerelated health care. The prevalence of first-ever stroke is increasing and in 2020 the expected prevalence will be $62 \%$ (compared to $41 \%$ in 1991)[1]. This increasing prevalence is related to aging of the population. For patients who survive, stroke can have tremendous consequences, both physically and mentally. It is only recently that the cognitive deficits due to stroke have received more atten- 
tion. Most studies on the relation between stroke and cognitive disorders have reported on vascular dementia $(\mathrm{VaD})$. The prevalence of $\mathrm{VaD}$ varies from $8 \%$ to $26 \%$, and the risk of developing $\mathrm{VaD}$ increases by 9-fold within 3 months of stroke [2-6]. However, the concept $\mathrm{VaD}$ has been questioned because it falsely implies a homogeneous entity with a single underlying pathology. The broader concept of vascular cognitive impairment (VCI) circumvents these problems [7]. In VCI, vascular refers to all causes of ischemic cerebrovascuar disease, while cognitive impairment encompasses all levels of cognitive decline. Only a few studies have investigated this concept [8-15] and of these studies, most focused on $\mathrm{VCI}$ as a predementia stage of $\mathrm{VaD}$ or studied $\mathrm{VCI}$ in terms of outcome measures such as institutionalization or mortality rates $[8,12,15]$. Few studies investigated the influence of risk factors on the development of VCI [11]. While it is accepted that new criteria should be evidence based and not consensus based, only a few studies have investigated the phenomenology of VCI. Moreover, it is unclear how the cognitive deficits progress with time and which risk factors cause deterioration. More needs to be learned about these gaps in our knowledge before criteria for VCI can be defined and used to identify patients at risk of cognitive deficits or of becoming demented. The CODAS (COgnitive Disorders After Stroke) study is described in this thesis.

\section{Aims and Outline of this thesis}

The main aim of CODAS was to investigate the profile of cognitive deficits after stroke. Moreover, the course of and risk factors for vascular cognitive deficits were also studied, the results of which are described in this thesis.

1) Background and methodology of CODAS

This thesis starts with a discussion of the concept Vascular Cognitive Impairment (VCI) (chapter II). The concept VCI was introduced because of difficulties in defining 'vascular' and 'dementia' when using the traditional concept of VaD. Chapter III deals with the methodological set-up of the CODAS study. This chapter also comments on the methodological choices made in CODAS regarding study design, procedure, patient population, clinical assessment (both neuropsychological and medical assessment), and how we dealt with missing data. The different diagnostic criteria used are also explained, such as for post stroke mild cognitive impairment and $\mathrm{VaD}$, and how we defined cognitive improvement or deterioration.

2) Profile and course of VCI

Chapters IV, V, and VI focus on the phenomenology of VCI and discuss epidemiological issues. In chapter 4 we investigated memory function, executive functioning, mental speed, and overall cognitive functioning after stroke. 
We wanted to expand the somewhat limited focus of studying only $\mathrm{VaD}$ after stroke, by investigating cognitive deficits not restricted to dementia. We described cognitive changes relative to cognitive functioning at earlier assessments. In chapter $\mathrm{V}$ we investigated the prevalence of cognitive disorders with time in patients who had been diagnosed with $\mathrm{VCI}$, defined as either $\mathrm{VaD}$ or post stroke mild cognitive impairment (MCI). In chapter VI we investigated whether the incidence of $\mathrm{VaD}$, in addition to its prevalence, is influenced by the choice of diagnostic system used.

The third part of this thesis concentrates on the prodromal stage of $\mathrm{VaD}$ and recovery from this stage. As VCI encompasses a continuum of cognitive deficits, from very mild to dementia, it should be possible to identify patients that are at risk of developing dementia. In chapter VII we investigated whether $\mathrm{MCI}$ has predictive value for the development of $\mathrm{VaD}$, as it does in Alzheimer's disease. In chapter VIII we used a stricter definition of recovery, compared to that used in earlier chapters, defining it in terms of recovery from a diagnosis 'permanent

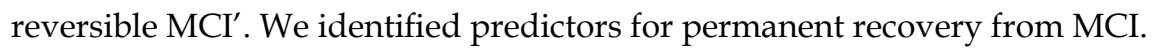

\section{3) Risk factors for VCI}

We investigated whether the vascular brain damage caused by stroke is related to $\mathrm{VCI}$ (chapter IX). A relation between different cognitive deficits (VaD or post stroke $\mathrm{MCI}$ ) and demographic or $\mathrm{CT}$ scan features was found. In chapter $\mathrm{X}$, we hypothesized that progressive silent brain infarction is related to cognitive deterioration in stroke patients. We compared the cognitive performance of patients with progressive brain damage with that of patients without progression.

4) Relation between VCI and psychiatric symptoms

In chapter XI we describe a screenings instrument which we developed for identifying both cognitive and emotional complaints after stroke. The role of psychiatric symptoms in cognitive functioning after stroke is described in chapter XII. Lastly, in chapter XIII we present a general discussion of the results presented in this thesis. 


\section{Vascular Cognitive Impairment}

a new concept

Adapted from:

Rasquin SMC, Verhey FRJ, Lodder J. Vascular Cognitive Impairment: een nieuw concept. Nederlands Tijdschrift voor Neurologie. 2003;104(4);209-214. 


\section{Abstract}

Although Vascular Dementia (VaD) is widely used its definition remains vague and its use problematic. In this context, the term 'vascular' has been poorly described and most criteria for $\mathrm{VaD}$ do not adequately reflect the underlying pathology. Moreover, the diagnosis $\mathrm{VaD}$ is established on the basis of the criteria for Alzheimer's disease, and thus overemphasises the importance of memory disturbances. These shortcomings led to the introduction of a new concept: vascular cognitive impairment (VCI), to describe cognitive disorders that are related to vascular damage in the brain. This chapter describes the concepts $\mathrm{VaD}$ and VCI, and their applicability in practice. 


\section{Introduction}

Stroke is, after Alzheimer's disease (AD), the main cause of dementia. It increases by 9-fold the risk of vascular dementia $(\mathrm{VaD})$, defined according to the Diagnostic and Statistical Manual third revised version (DSM III R) 3 months after stroke [6]. The prevalence of $\mathrm{VaD}$ among stroke populations varies from $6 \%$ to $26.3 \%$ [2, 16-18]. The broader concept 'vascular cognitive impairment' (VCI) was recently introduced by Bowler and Hachinski [7] to describe a profile of cognitive deficits related to vascular brain damage. $\mathrm{VCI}$ encompasses three subgroups: 1) VCI without dementia; 2) $\mathrm{VCI}$ in which the criteria for $\mathrm{VaD}$ are met; and 3) VCI in which criteria for other forms of dementia (mixed form) are met. Thus VCI includes not only dementia, but also milder forms of cognitive deterioration. $\mathrm{VCI}$ without dementia is more common than $\mathrm{VaD}$ as a sequel of cerebrovascular disease $[12,18]$. In this chapter, we explain why there is a need to enlarge the concept $\mathrm{VaD}$, and discuss the implications of the concept $\mathrm{VCI}$ for research and clinical practice.

\section{Problems with the concept 'Vascular Dementia'}

$\mathrm{VaD}$ has a rich past, a confusing present, and an uncertain future [19]. In defining VaD, the terms 'vascular' and 'dementia' both prompt questions. 'Vascular' refers to the cause of the dementia syndrome. This term has been changed several times. In 1974, Hachinski et al. introduced the term 'multi-infarct dementia' (MID) [20]. Although the term suggests that only multiple infarctions give rise to dementia this is not completely correct because, other cerebrovascular mechanisms, such as a strategic infarct or white matter lesions, can also cause cognitive deterioration [21]. So, different vascular mechanism can be etiologically related to dementia, and as such the concept $\mathrm{VaD}$ is a heterogeneous one.

The DSM and the 'International Classification of Diseases' tenth version (ICD10), formulated, the etiopathology of VaD more clearly [22, 23]. For instance, in the last version of the DSM one of the criteria requires evidence of cerebrovascular brain damage. Thus indicators of cerebrovascular damage, such as focal neurological signs or symptoms, or laboratory evidence have to be etiologically related to the cognitive deficit. The DSM definition also includes different cerebrovascular mechanisms. The ICD-10 is even more specifically and defines different forms of $\mathrm{VaD}$ : MID, subcortical $\mathrm{VaD}$, and mixed cortical and subcortical VaD. However, in both diagnostic systems it remains unclear how the relation between the different etiological mechanisms and the development of dementia should be operationalized. In practice, the diagnosis VaD depends on the opinion of the clinician. 
These shortcomings have led to the formulation of consensus-based criteria, such as those of the 'State of California Alzheimer's Disease Diagnostic and Treatment Centers' (ADDTC), and those of the 'National Institute of Neurological Disorders and Stroke/Association Internationale Pour La Recherche et l'Enseingement en Neurosciences' (NINDS-AIREN) [24, 25]. These criteria are more explicit than the DSM and the ICD-10 in describing the underlying pathology of $\mathrm{VaD}$. One of the criteria of the ADDTC makes it possible to identify different subtypes of $\mathrm{VaD}$, based on infarct characteristics such as size, location, and severity and cause of ischemic brain damage [25]. The NINDSAIREN criteria also describe different mechanisms of ischemic brain damage that could give rise to $\mathrm{VaD}$, such as multiple infarcts, a strategic infarct, lacunar infarcts, extensive white matter lesions, or a combination [24]. Both classification systems incorporate two new elements, a temporal relation between the vascular damage and the dementia syndrome, and the necessity of neuroradiological investigation. Moreover, the criteria give an indication of the probability that clinical signs reflect a neuropathological substrate.

Thus, the term 'vascular' describes heterogeneous mechanisms. This heterogeneity may explain why the prevalence of $\mathrm{VaD}$ varies in different studies and why some medication trials have yielded 'negative' results [26]. It is important to form more homogeneous subtypes of $\mathrm{VaD}$, as recently proposed by Erkinjuntti et al., who introduced the concept 'subcortical $\mathrm{VaD}^{\prime}$ ' 26]. This concept covers the condition formerly known as 'Binswanger's disease' and 'Lacunar State'. Subcortical $\mathrm{VaD}$ is characterized by deficits in executive functioning, such as planning, goal formulation and organization, mild memory-deficit, multiple depressive episodes, and personality changes and/or emotional lability [27]. Although the consensus- based criteria are an improvement, they still have some shortcomings in clinical practice. The mixed form of dementia (a combination of $\mathrm{VaD}$ and $\mathrm{AD}$ ) receives little attention, despite there being increasingly more evidence that the risk factors for $\mathrm{VaD}$ and $\mathrm{AD}$ overlap [28]. Moreover, in most patients with $\mathrm{AD}$ there is also some form of ischemic brain damage present and, conversely, in patients with $\mathrm{VaD}$ there is often some degree of brain atrophy. To date, there are no methods available that distinguish mixed forms of dementia from 'pure' VaD.

In addition to the problem of defining 'vascular', there is the problem of defining 'dementia'. A major problem is that the diagnosis of dementia is based on that of $\mathrm{AD}$, in which a memory disorder is a requisite. Thus problems in the performance of daily activities due to a cognitive deficit other than memory are not taken into account. In a study of stroke patients, we found that although $22 \%$ of the patients had a memory deficit 1 month after stroke, as many as $50 \%$ had a diminished mental speed [29]. Moreover, because the phenomenological criteria of $\mathrm{VaD}$ are based on those of $\mathrm{AD}, \mathrm{VaD}$ would appear to be a homoge- 
neous entity, which is not. Third, the criterion that a cognitive deficit should lead to interference with daily activities is problematic in patients who have had a stroke, because it is difficult to judge whether the interference is due to physical handicaps or due to cognitive deficits.

The terms 'vascular' and 'dementia' are not only difficult to describe, but the temporal relation between them is often difficult to establish. Because of the acute nature of a stroke, the temporal relation is clear in most instances, however this is not the case if there are only white matter lesions or silent infarcts. Moreover, if there are several cerebral lesions, it is difficult to define which one is most important in terms of cognitive deficit [30]. Lastly, it should be pointed out that many healthy elderly people have vascular lesions of one sort or another on imaging [31].

\section{Vascular Cognitive Impairment}

Because of dissatisfaction with the concept VaD, Bowler and Hachinski introduced the concept 'vascular cognitive impairment' [7]. This concept is not solely restricted to the diagnosis $\mathrm{VaD}$, but encompasses all forms of cognitive impairment. Thus VCI is more prevalent than VaD. Tatemichi et al. found that $35.2 \%$ of stroke patients had cognitive deficits on at least four cognitive domains, whereas only $3.8 \%$ of healthy controls did [32]. Pohjasvaara et al. found prevalence rates as high as $61.7 \%$ among stroke patients [2], and Rockwood et al. reported that $25 \%$ of patients attending a memory clinic were diagnosed as having $\mathrm{VCI}$, of which $25 \%$ had $\mathrm{VaD}$ [11].

The most important reason for formulating a new concept is because, accor-ding to current criteria, $\mathrm{VaD}$ can be diagnosed only if cognition has deteriorated so much that daily activities are affected. Because it may be possible to prevent cerebrovascular disease, and consequently cognitive deterioration, it is desirable to diagnose cognitive deterioration before dementia develops. The new concept explicitly focuses on this early stage of cognitive deterioration.

As in studies of 'mild cognitive impairment' (MCI) in AD research, the question arises as to the prognostic value of the concept VCI. In a population survey, Rockwood et al. found that people with VCI had a poor prognosis compared with that of cognitively healthy individuals, people, diagnosed with VCI were more likely to be institutionalized or to die (RR 3.1 and 1.8 respectively) [12]. Wentzel et al. found that $50 \%$ of patients with VCI without dementia, became demented after 5 years [15]. In addition, there is the question whether specific cognitive deficits have prognostic value. Ingles et al. found that the prodromal stage of $\mathrm{VaD}$ is characterized by difficulties with fluency tasks and memory tasks [10]. Meyer et al. pointed out that damage to the subcortical small vessels, 
in contrast to a strategic infarct or multiple cortical infarcts, leads to cognitive deterioration, which could be described as the prodromal stage of dementia [33]. Thus it is probably that a prodromal stage is present in only one subtype of $\mathrm{VaD}$.

Despite the advantages of the concept VCI, some issues remain unresolved. First, it is not clear how 'cognitive deficit' should be defined. In most cases a cognitive deficit is diagnosed if patients perform worse than a normative group. However, stroke patients are usually older (70 years and older) and there are few normative data for these age ranges. Cognitive decline can also be defined as decline from an earlier level, which is usually established retrospectively and as such is subjective. A second problem is that silent infarcts and white matter lesions could also contribute to the development of VCI, but a causal relation between these mechanisms and VCI is difficult to establish. A last problem is that with the introduction of the concept $\mathrm{VCI}$ it is no longer possible to categorize subtypes of $\mathrm{VCI}$, as was the case with $\mathrm{VaD}$. Because clinicians tend to work in terms of disease subcategories, in research settings this lack of VCI criteria may lead to the creation of artificial boundaries in order to define subgroups of VCI.

In summary, the concept $\mathrm{VCI}$ is preferable to that of $\mathrm{VaD}$, but it is too early to determine its relevance in clinical practice. Bowler and Hachinski stressed that it is premature to formulate formal criteria because too little is known about cognition in cerebrovascular disease [7].

Future research should focus on risk factors for VCI. The prognostic value of $\mathrm{VCI}$ has to be investigated and also the possibilities for prevention and treatment. The longitudinal study CODAS, which started in 2000 with inclusion of patients from the Maastricht stroke unit, will answer some of these questions [29].

\section{Conclusion}

According to the current criteria of $\mathrm{VaD}$, first dementia is diagnosed, based on the criteria for $\mathrm{AD}$, and then $\mathrm{VaD}$ is diagnosed, based on the presence of cerebrovascular damage. This method does not take into account the differences in cognitive profiles and underlying pathologies between $\mathrm{VaD}$ and $\mathrm{AD}$. The concept $\mathrm{VaD}$ has been revised several times, but even the most recent criteria can not give a satisfactory answer to the question 'what is dementia caused by vascular brain damage'. While the concept VCI resolves some of these problems, it does not resolve them all. People with vascular brain damage are at greater risk of cognitive deterioration, be it a mild form or dementia, but it is not yet clear over what time frame and with what type of brain damage. 


\section{III}

Design and outline of CODAS 


\section{Design}

The COgnitive Disorders After Stroke (abbreviated as CODAS) study is a longitudinal prospective study investigating the presence of cognitive disorders and their development after stroke. Patients entering the study were consecutive patients who had experienced a first-ever cerebral stroke and who attended the department of Neurology or the outpatient stroke unit of the hospital. The patients were followed up for 2 years with clinical evaluations at 1, 6, 12, and 24 months post-stroke. This chapter describes the design and outline of the CODAS-study.

\section{Procedure}

The Medical Ethics Committee of the University Hospital Maastricht approved the project. Patients were asked within 48 hours after stroke to participate. All patients were given verbal and written information about the study and gave their written consent to participation. They were clinically assessed at 1, 6, 12, and 24 months post-stroke. either at home or in the hospital. The assessment took about 1,5 hour. Afterwards, caregivers were asked to fill in the questionnaires.

After each assessment, data were entered in the CODAS database and the patients were sent a standard letter about their test performances. The family doctor was informed if patients scored lower than 24 out of 30 on the Mini Mental State Examination (MMSE) or 79 out of 105 on the CAMCOG. Request for the follow-up scan was made at the last assessment.

\section{Patients}

Patients were recruited between January 2000 and June 2001. In this period, 592 patients who had been admitted to the Department of Neurology, or who contacted the outpatient clinic of the University Hospital Maastricht were screened for eligibility for participation in the CODAS study.

Inclusion criteria were: 1) First-ever stroke not located in the cerebellum or brainstem. This was because it is unclear whether cognitive performance is influenced by stroke in one of these locations. 2) Age older than 40 years, to exclude patients with atypical stroke. 3) Adequate post-stroke fluency in Dutch, which is a requisite for valid neuropsychological testing. 4) An initial MMSE score higher than 15 to ensure that testing was possible. Exclusion criteria were: 1) Severe aphasia (diagnosed by a speech therapist). This is an exclusion criterium for the 
diagnosis vascular dementia in the National Institute for Neurological Disorders and Stroke, Association Internationale pour la Recherche et L'Enseigement en Neuroscience (NINDS-AIREN) criteria [24]. 2) Comorbid neurological disorders other than the qualifying event, such as a tumour. And 3) major psychiatric disorder as reported in the patient's medical files.

Of the 592 patients, 346 did not met the inclusion or exclusion criteria: 89 were not first-ever strokes, 57 had a lesion located in the brainstem or cerebellum, 80 died within 1 month of the stroke, 9 were younger than 40 years, 5 were not native Dutch speaking, 6 were in a coma, 46 patients had an MMSE score lower than 15, 34 had severe aphasia, 20 had other neurological or psychiatric disorders. Of the 246 patients eligible for participation, 35 patients refused to participate, 6 were admitted too long after their stroke so that the first assessment could not take place, and 9 lived too far from the hospital.

Patient characteristics are presented in table 1 . Age, sex, Rankin score before and after stroke $(0=$ no complaints; $1=$ complaints no restrictions; $2=$ some restrictions, no help needed; $3=$ help needed, mostly independent; $4=$ mostly needy; 5 $=$ constant nursing needed) and Canadian Neurological Scale (CNS) (measurement including consciousness, orientation, speech, face, strength in arm and leg) were recorded for both the included and the excluded patients. The total score of the CNS (non-categorical range) is 10, indicating no neurological deficits [34, 35].

\begin{tabular}{llll}
\hline Table 1: Patient characteristics & & & \\
\hline & Included & $\begin{array}{l}\text { Excluded/ } \\
\text { otherwise }\end{array}$ & Refused \\
\hline $\mathrm{N}$ & 196 & 361 & 35 \\
Age (mean, SD) & $68.2(12.6)$ & $71.6(13.9)$ & $71.6(11.8)^{*}$ \\
Sex (M-F) & $107-89$ & $185-176$ & $23-12^{*}$ \\
Rankin before stroke (mode) & $0(58.2 \%)$ & $0(39.9 \%)$ & $0(60.0 \%)^{*}$ \\
Rankin post-stroke (mode) & $3(29.6 \%)$ & $5(33.0 \%)$ & $3(37.1 \%)^{*}$ \\
$\begin{array}{l}\text { Canadian Neurological Scale } \\
\text { (mean, SD) }\end{array}$ & $8.1(1.8)$ & $6.8(2.9)$ & $8.4(1.2)^{*}$ \\
\hline${ }^{*} \mathrm{p}<.05$ & & & \\
\hline
\end{tabular}

All patients, both those who participated and those who refused participation, were asked to complete the Cognitive Screenings Test by telephone [36]. Twenty-four $(68.6 \%)$ excluded patients complied. There were no differences in test scores between the patients who were included (mean $=17.4, \mathrm{SD}=2.5)$ and the patients who refused participation (mean $=17.8, \mathrm{SD}=1.9)$. The maximum score was 20 . 


\section{Clinical assessment}

Patients were tested at 1, 6, 12, and 24 months post-stroke by a neuropsychologist.

\section{Clinical Interview}

Semi-structured Interview: This questionnaire is based on the diagnostic criteria for dementia proposed by the DSM IV [23]. In an interview with the caregiver, the cognitive performance of the patient was assessed by means of a semi-structured questionnaire. The caregiver was asked whether the patients had memory problems, or other cognitive impairments (i.e.language, executive, agnosia, and apraxia) before stroke. If present, the caregiver was asked to indicate how much these impairments interfered with daily activities. Caregivers were also asked whether the patient had shown any signs of dementia before the stroke. This interview was repeated at each assessment; however, after the first assessment the focus was no longer on cognitive functioning before stroke but on cognitive functioning relative to that at the previous assessment.

Symptom Check List: This questionnaire is a multi-dimensional self-rating scale and covers a range of psychiatric symptoms, such as anxiety, agoraphobia, depression, somatic complaints, interpersonal sensitivity, insufficiency of thinking, sleeping problems, and hostility [37]. The questionnaire was chosen because it is short and answers are checked, i.e. it does not require patients to write. Patients were asked to indicate, on a 5-point scale, how much hinder they had experienced from certain psychiatric symptoms in the last week. If patients were not able to complete the questionnaire by themselves, we asked the caregiver to help them.

Interview for Deterioration in Daily life in Dementia: This questionnaire (abbreviated as IDDD) was completed by the caregivers [38]. The questionnaire concerns self-care and complex activities, with attention being paid to lack of initiative and impaired performance. On a 4-points rating scale, the caregiver rated the amount of initiative the patient still showed and the amount of help the patient needed. In $3.6 \%$ of the cases there was no caregiver present, and the neuropsychologist filled in the IDDD in consultation with the patient. These patients lived independently and did not need help. 


\section{Medical Assessment}

Medical information (presence of diabetes, hypertension, cholesterol, hart failure in the past, family history of vascular diseases, neurological functioning, and stroke diagnosis) was collected on admission and entered into the Maastricht Stroke Register (MSR), a prospective databank. This databank contains information on all stroke patients treated at the University Hospital of Maastricht.

\section{Neuropsychological Tests}

CAMCOG: This is the cognitive part of the Cambridge Examination for Mental Disorders of the Elderly (CAMDEX), a standardized instrument for the diagnosis and gradation of dementia in the elderly [39]. The CAMCOG can be subdivided into 10 subscales: orientation, language-expression, language-understanding, memory (long and short term), attention, calculation, praxis, abstract thinking, and abstract perception. The CAMCOG is well tolerated by patients and is sensitive to cognitive disorders in stroke patients [40]. The CAMCOG includes the items of the MMSE [41].

Auditory Verbal Learning Test: The Auditory Verbal Learning Test is an episodic memory test [42]. Briefly, 15 words are orally presented one after another, separated by short time intervals. Then the subject is asked to recall as many words as he/she can, in his/her own order. This procedure is repeated five times and after 20 minutes, delayed recall and recognition are tested.

The test outcome is the number of correctly recalled words after each trail and after the delay. We used this test to assess memory because it draws on both working memory and episodic long-term memory.

Concept Shifting Task: The Concept Shifting Task (CST) is used to measure visual conceptual and visuomotor tracking $[43,44]$. The CST consists of three parts; each part is presented on a test-sheet on which 16 circles are grouped in a larger circle. In the small circles the test-items (part A numbers; part B letters; part $C$ both) appear in a fixed random order. Subjects are requested to cross out the items in the right order (increasing order in for parts A and B, and alternating between the letters and the numbers in increasing order in part $\mathrm{C}$ ). The time needed to complete the three parts is the variable of interest. We used this test to measure visual-conceptual and visuomotor tracking because it is simple to administer and subjects do not have to write.

Stroop Colour Word Test: The Stroop Colour Word Test (SCWT) is a test of selective attention and interference susceptibility [45]. The SCWT involves three cards displaying a hundred stimuli each: colour names, coloured patches, and colour names printed in incongruously coloured ink. In the first part, the patient has to read the printed names of the colours; in the second part the patient has 
to name the coloured patches; and in the last part the patient has to name the colour of the ink. The variable of interest is the time needed to complete the test. We chose this test because it is widely used and it is also applicable for patients with head trauma [46].

Groninger Intelligence Test: the Goninger Intelligence Test (GIT) is a test of general intelligence [47]. The subscales used were: 'doing sums', which involves the correct completion of as many sums (addition) as possible in 1 minute, and 'mental rotation', which requires the subject to indicate which two-dimensional shapes from a large set are needed to exactly fill up a given space on the test page. These subscales were used to test the cognitive domains calculation and visuospatial functioning. The tests can be completed by stroke patients because there is little writing involved.

All neuropsychological tests had a high reliability (minimum was 0.81) [46].

\section{Cognitive Domains}

Eight cognitive domains were evaluated, based on the criteria for $\mathrm{VaD}$ of the NINDS-AIREN [24]: memory, executive functioning, orientation, attention, language, praxis, visuospatial, and calculation (Table 2). In addition, mental speed was also included as a cognitive domain.

Compound scores were calculated for 'memory', 'mental speed', and 'executive functioning' (see table 2). Patient scores on a given test were converted to standardized scores with the following formula: $Z=$ (patient-score - norm score)/ standard deviation from the norm group. The mean of these z-scores for each separate cognitive domain was calculated. The reliability of the compound scores was calculated using the following formula [(mean reliability of the separate test $+(\text { number of separate test }-1)^{*}$ correlation between tests) $] /[1+$ (number of separate tests -1$)^{*}$ correlation between tests] [48]. The reliability of the memory compound score was 0.91 , that of the mental speed compound score was 0.90 , and of the executive functioning compound score was 0.53 . 


\begin{tabular}{ll}
\hline \multicolumn{2}{l}{ Table 2: Cognitive Domains } \\
\hline$\underline{\text { Domains }}$ & $\underline{\text { Tests }}$ \\
Memory & AVLT, total words direct recall and delayed recall \\
Mental Speed & SCWT I, CST 0, I and II \\
Executive Functioning & Mean interference score SCWT, CST \\
Calculation & GIT, doing sums \\
Visuospatial & GIT, mental rotation \\
Orientation & CAMCOG items 139-148 (place, person, time) \\
Attention & CAMCOG items 178-179 (serial 7's, counting backward) \\
Praxis & CAMCOG items 183-186/188-189/191-193 \\
& (copying, ideational, ideomotor) \\
Reasoning & CAMCOG items 197-200, 201-203 \\
& (similarities, perception) \\
Language & CAMCOG items 149-163/181-182 \\
& (understanding, expression, writing) \\
\hline AVLT: Auditory Verbal Learning Test | SCWT: Stroop Colour Word Test | \\
CST: Concept Shifting Test | GIT: Groninger Intelligence Test | MMSE: Mini \\
Mental State Examination & Interference-score: Part III - mean (Part I + part II) \\
\hline
\end{tabular}

\section{Cognitive Deficit}

The neuropsychological performance of individual patients was compared with normative data derived from the MAastricht Aging Study (MAAS) [49]. The aim of the MAAS-study is to identify determinants of normal cognitive aging among healthy older volunteers living in the south of the Netherlands. Normative data from this study were stratified according to age, sex, and educational level. Educational level was classified as 'low' (primary education and vocational education), 'middle' (secondary school), and 'high' (all other educational levels). Individual test scores were converted to standard Z-scores, in order to define cognitive deficits, using the means and standard deviations of the normative data (test score patient - mean score norm group)/SD norm group. A cognitive deficit was defined as a score lower than the 10the percentile of the z-scores of the norm group (this is equal to a standard deviation of 1.28).

For the CAMCOG, a cognitive deficit was defined as a score lower than $75 \%$ of the total score possible for each subscale. This is comparable to the cut-off of 79 out of 105 of the total scale, which indicates a cognitive deficit [50]. 


\section{Diagnosis Dementia}

Two experienced clinicians (a neuropsychiatrist and a neuropsychologist) diagnosed, independently from each other, dementia according to the DSM-IV criteria. They were blind to data from the previous assessment and made the diagnosis based on the patient's medical history, a structured interview with an informant, clinical observation, and the patient's test performance. Interference with daily activities caused by cognitive deficits was assessed with the Interview for Deterioration in Daily life in Dementia (IDDD) [38]. The diagnosis of VaD was based on the NINDS-AIREN criteria [24]. If there was disagreement, the final diagnosis was negative. Agreement between the two raters was excellent (Kappa, $\mathrm{K}=0.88 ; \mathrm{p}=.00$ ).

\section{Missing Data}

Neuropsychological testing in patients with stroke is not without complications - some patients can not perform tests because of paralysis of the arm or speech problems. Consequently, test data were incomplete (up to $28 \%$ were missing). To perform valid analyses with sufficient power, missing data were imputed according to the standard procedure described by Tabachnick et al. [51]. Imputation was only performed if data were available for at least one other test for the same cognitive domain and if the missing test was administered at an earlier or later assessment. An experienced neuropsychologist, blind to the outcome, decided whether and how to impute the missing data, based on the patient's cognitive profile and available test data. Imputation can also be based on regression analysis. We used this method for the first assessment in order to validate one method against the other. The means and standard deviations of the test scores did not differ between the databases without imputed data or with data imputed according to one of the two methods, indicating that the two methods were comparable. So, imputation increased the power of the data without affecting the conclusions draw. We chose for imputation by an experienced neuropsychologist so that the patient's entire neuropsychological profile could be taken into account. Table 3 presents the number of missing data and the percentages of this missing data that was imputed. 


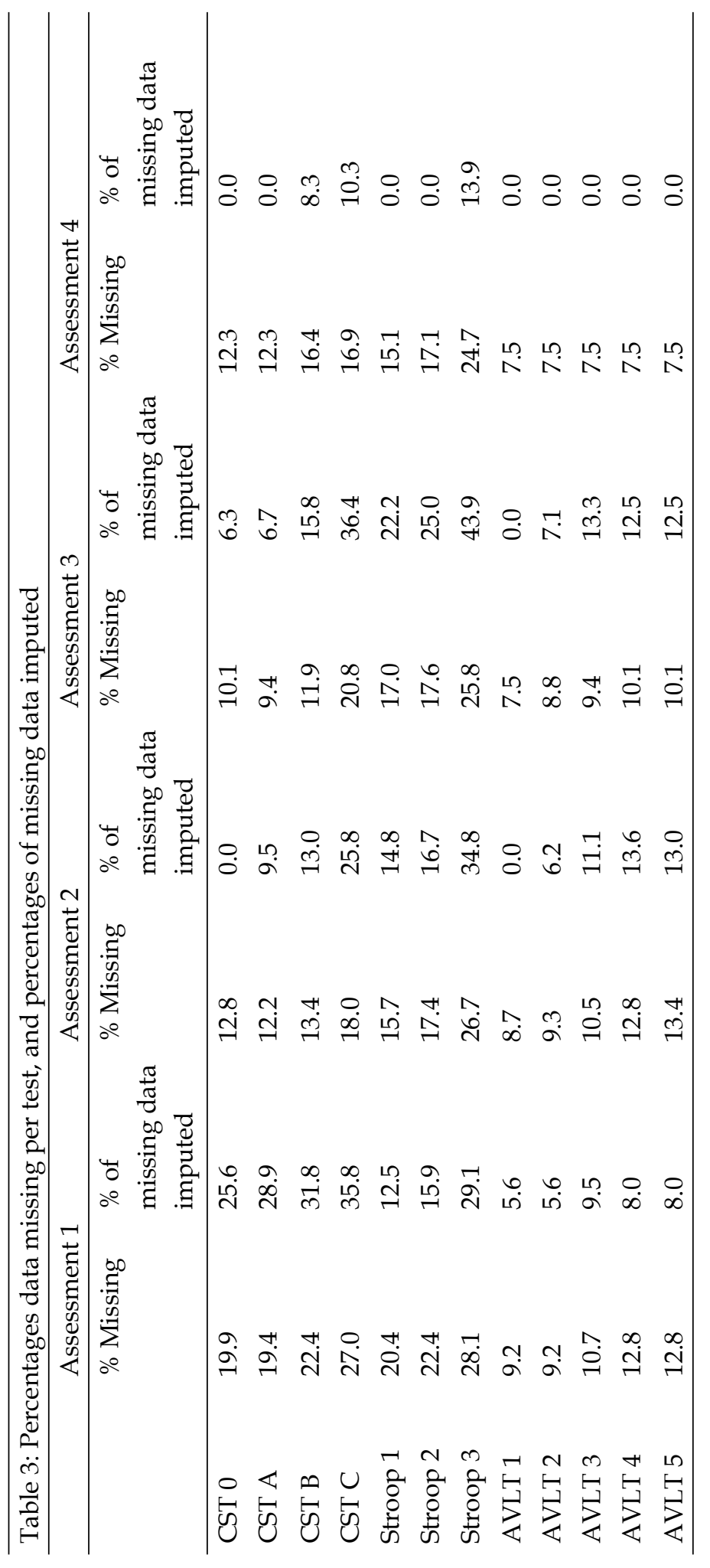




\section{Brain Imaging}

Although magnetic resonance imaging (MRI) scan is more sensitive than computed tomography (CT) for detecting brain damage, we used CT for two reasons. First, access to the MRI facilities was limited at the University Hospital Maastricht during the study period. Secondly, almost all stroke patients underwent $\mathrm{CT}$ in the acute phase of stroke. Moreover, it is uncertain whether MRI is more specific than $\mathrm{CT}$ in determining whether a small lesion is ischaemic. Even in the absence of a specific lesion on CT, lacunar and territorial infarctions can be distinguished accurately on clinical grounds [52]. All patients had at least one brain CT scan. In most patients, CT was performed at admission or 1 day later (52.9\%; mode $=1$ day, range of 0 to 150 days). If patients had a second scan on which the specific lesion was seen more clearly, this scan was used. The scans of 11 patients $(5.6 \%)$ could not be retrieved from the archives of the Department of Radiology.

A neurologist recorded the following features: side of the symptomatic infarct or haemorrhage (left or right hemisphere); lacunar or territorial ischemic stroke; presence of white matter lesions; presence of silent infarcts; and brain atrophy. For definitions we refer to de Jong et al.[53]. White matter lesions were defined as focal or diffuse hypodensities in the periventricular or deep white matter, not involving the cortex, with ill-defined margins [54]. White matter lesions were further specified by site: around the frontal or posterior ventricle horns, or paraventricular/centrum semi-ovale area. No effort was made to quantify the density of the WML in these different sites, because this might depend on the tuning of the scanning and a certain variability related to the procedure of making the scan hard-copy. Silent brain infarction was defined as a low-density area on the $\mathrm{CT}$, compatible with infarction but without a history of stroke based on the patient's history or any other accessible information. Also, the stroke symptoms at study entry had to be anatomically incompatible with such silent infarcts. Silent infarcts or white matter lesions, or both, were defined as 'prestroke cerebrovascular damage'. The degree of atrophy was scored in a semi-quantitative way (none, mild, moderate, and severe), according to the criteria of Leys et al [55]. In short, the CT scans of four patients were selected during a consensus meeting by three experts to serve as examples of the four categories of brain atrophy (no, mild, moderate, or severe). CT scans of study patients were graded by visual comparison with the example scans. If no lesions were visible on $\mathrm{CT}$, clinical data were used to categorize the patients, as having lacunar or territorial brain infarction.

Two neurologists examined the CT scans independently. They were unaware of the neurological signs and the neuropsychological data. Inter-rater agreement in the classification of patients by stroke features on CT was excellent, with a $\mathrm{K}$ 
of $0.88(\mathrm{p}=.00)$ for symptomatic side of the infarct, a $\mathrm{\kappa}$ of $0.94(\mathrm{p}=.00)$ for the symptomatic side of the haemorrhage, a $\mathrm{\kappa}$ of $0.85(\mathrm{p}=.00)$ for location, a $\mathrm{\kappa}$ of 0.60 $(\mathrm{p}=.00)$ for white matter, a $\mathrm{K}$ of $0.79(\mathrm{p}=.00)$ for silent infarcts, and a $\mathrm{K}$ of 0.69 $(\mathrm{p}=.00)$ for atrophy.

Two years after stroke, patients underwent repeat CT scanning, to study whether the brain damage had increased and to relate this to cognitive functioning. New lesions, visible on the CT scan compatible with infarction were considered to reflect new infarcts, either silent or symptomatic. White matter lesions were considered to have increased in size if patients showed new lesions that were not present at baseline, in at least one of the three distinguished areas: around the frontal or posterior ventricle horns, or the para-ventricular/centrum semi ovale areas.

Of the 143 patients available at the last measurement, 101 participated in this part of the study. Thirteen patients could not come to the hospital because of transport problems, 15 patients refused further participation, 5 patients were too ill to participate, 2 had patients died, 2 patients were demented at baseline, and 5 patients did not have a baseline scan.

\section{Attrition}

One month after stroke 196 patients were included in CODAS. At 6 months after stroke 173 patients were re-assessed, 4 patients had died, 14 refused further participation, 4 patients refused only for this assessment, and 1 patient was untraceable. At 12 months after stroke 157 patients were re-assessed: 8 patients had died (between the second and third assessments), 6 refused further participation, 4 patients refused for this measurement, and 2 patients were too ill. At 24 months after stroke 143 patients were re-assessed; 15 had died (between the third and fourth assessment), 4 patients refused further participation, and 1 patient was too ill to come for assessment. Patients who completed the study were younger (mean age at baseline 65.8; sd 12.1) than those who did not (mean age 75.6; sd 10.8). The mean baseline MMSE score was higher in the patients who completed the study (mean 24.1; sd 3.8) than in the patients who did not (mean 25.9; sd 3.4). Sex was equally distributed in the two groups. However, patients who completed the study were higher educated $(51.0 \%$ had a low educational level) than the patients who did not (70.6\% had a low educational level). 


\section{Vascular Cognitive Disorders: memory, mental speed and cognitive flexibility after stroke}

Adapted from

Rasquin SMC, Verhey FRJ, Lousberg R, Winkens I, Lodder J. Vascular cognitive disorders. Memory, mental speed and cognitive flexibility after stroke. Journal of the Neurological Sciences. 2002;203-204;115-119. 


\section{Abstract}

Background: This study investigated the occurrence of cognitive disorders 1 and 6 months after stroke in a cohort of patients with a first-ever stroke. In addition, it was investigated whether age, sex, and level of education are risk factors for vascular cognitive disorders.

Methods: Memory, simple speed, cognitive flexibility, and overall cognitive functioning were examined in 139 patients at 1 and 6 months post-stroke. Inclusion criteria on admission were first cerebral stroke, age $\geq 40$, no other neurological or psychiatric disorders and ability to communicate.

Results: Mean age was 69.3 years $(S D=12.3)$. Patients were compared with a healthy control group matched for age, sex, and level of education. A large group of patients who, at 1 month after stroke, scored below the cut-off on cognitive domains, scored above the cut-off on most of these cognitive domains at six months. For overall cognitive functioning 16 out of 39 , for memory 13 out of 26 and for cognitive flexibility 15 out of 49 patients, who at 1 month scored below the cut-off, scored above the cut-off at 6 months. Simple speed did not change; 12 patients scored above the cut-off and 7 patients scored below the cutoff at 6 months after stroke. Speaking in terms of improvement or deterioration, most people remained stable on the four cognitive domains (ranging from $37.6 \%$ to $83.5 \%$ ), and a substantial group improved (ranging from $12.9 \%$ to $52.1 \%$ ).

Conclusions: Older and female patients had more cognitive disturbances. Overall the conclusion is that the prognosis of cognitive functioning after stroke is general favourable, especially in younger patients. 


\section{Introduction}

Although stroke is a major neurological disorder, often leading to serious and long-lasting sensorimotor and behavioural disabilities, little is known about the severity and frequency of cognitive impairments following stroke. However, studying the development of post-stroke cognitive disorders is of prognostic value. Moreover, it is important to identify vascular cognitive impairment at an early stage, because of the possibility to prevent further cognitive decline [56]. Although this view is fully accepted, cognitive functions are not routinely assessed shortly after stroke $[13,32,57]$.

Most publications $[58,59]$ on neuropsychological consequences of stroke used only limited and global cognitive screening tests like the Mini Mental State Examination (MMSE) [41] or the simple Mental Test Score (MTS) [60]. However, such tests do not allow conclusions about specific functions. Other studies focused on isolated impairments such as neglect, memory or visuo-spatial disturbances $[57,61]$, or used a categorical approach by examining dementia as the major outcome $[2,6,13,16,62-64]$. An alternative approach to characterize the cognitive consequences of stroke would be to examine a broader range of specific neuropsychological functions, independent of the diagnosis of dementia. The aim of the present study was to examine the occurrence and course of cognitive disorders at 1 and 6 months after stroke in a cohort of patients with a first-ever stroke, and to identify demographic variables of influence on these cognitive disorders.

\section{Methods}

\section{Patients}

Patients enrolled in the study were participants of a larger study, CODAS (COgnitive Disorders After Stroke). CODAS is a prospective, observational study in which patients with a first-ever cerebral stroke are tested neuropsychologically. The main purpose of CODAS is to study the development of cognitive disorders after stroke and to identify risk factors that are related to the cognitive decline.

Inclusion criteria were first-ever cerebral stroke, age older than 40 years (to exclude atypical stroke), adequate fluency in Dutch, and MMSE score higher than or equal to 15 . Stroke was diagnosed clinically by the neurologist on duty, supported by routine investigations such as computed tomography. Exclusion criteria were other neurological or psychiatric disorders, which could lead to cognitive impairment. 
Consecutive stroke patients admitted to, or seen at the outpatients' clinic of the University Hospital Maastricht between January 2000 and July 2001 entered the study. Patients were asked within 48 hours after stroke to participate. Clinical information was collected on admission, and entered into the Maastricht Stroke Register (MSR), a prospective databank containing clinical information of all stroke patients treated in the University Hospital of Maastricht. Each patient was tested within 1 month after stroke and at 6 months after stroke. In total 196 patients were included in the study. Data for the 6-months assessment were available for 139 patients (79 men and 60 women; mean age $69.3 \pm 12.3$ years; mean MMSE score $25.6 \pm 3.5$ ). Cognitive functioning was assessed using a neuropsychological test battery (see below). Because of physical or mental handicaps not all patients were able to complete the whole test battery. The number of patients performing the tests is given in Table 1.

Patients were compared with a control group from the MAastricht Aging Study (MAAS). This population-based study focuses on determinants of normal cognitive aging. The study covers the full adult lifespan and is characterized by a cross-sequential design and in-depth neurocognitive and biomedical evaluation of a group of initially healthy individuals [49]. Normative data from this study were stratified according to age, sex, and level of education.

The Medical Ethics Board of the University Hospital Maastricht approved the protocol, and all participants gave informed consent.

\section{Neuropsychological assessment}

The tasks are described briefly. For detailed information see Jolles et al. [49]

Auditory Verbal Learning Test: This test is used to measure episodic memory. After presenting 15 words verbally to the patient, he/she is asked to recall as many words as he/she can remember. This procedure is repeated 5 times and after 20 minute delayed recall and recognition are tested [42].

Concept Shifting Task: This test is derived from the Trail Making Test and is used to measure the ease of shifting between two concepts [43]. There are three tasks in which the patient has to cross out 16 circles containing only digits (1 -16; part I), letters (A - P; part II) or both (1-A, 2-B, 3-C etc; part III). The variable of interest is the time needed to complete each subtest.

Stroop colour word test: The Stroop test is a test of selective attention and interference susceptibility. The test involves three cards displaying 100 stimuli each: colour names, coloured patches and colour names printed in incongruously coloured ink. For the last card the colour of the ink has to be named instead of the colour name. The variable of interest is the time needed to complete each subtest [45].

CAMCOG: This is the cognitive part of the Cambridge Examination for Mental 
Disorders of the Elderly (CAMDEX), a standardized instrument for the diagnosis and gradation of dementia in the elderly [65]. The CAMCOG examines cognitive functions, such as language, memory, praxis and abstract thinking, using several short tasks, which can be divided into several subscales. The Dutch revised version of the CAMCOG was used [50].

\section{Statistical Analyses}

The number of neuropsychological variables was reduced to four, by means of composite scores, namely memory (episodic memory), simple speed (routine basic cognitive functions such as reading), cognitive flexibility (speed of mental processing), and overall cognitive functioning. Memory was assessed by means of the Auditory Verbal Learning Test, maximum number of words recalled and number of words recalled after the delay. Simple speed was assessed by means of basic speed of Concept Shifting Test I and II, and reading speed of Stroop I. Cognitive flexibility was assessed by means of Stroop III and Concept Shifting Task III. Overall cognitive functioning was assessed by means of the CAMCOG score.

In order to form the composite scores, individual test scores were converted to standard Z-scores, using the means and standard deviations of the normative data of the MAAS study. Impairment was defined as a score below the 10th percentile because this cut-off is commonly used in clinical practice. Impairment on the CAMCOG score was defined as a score lower than 79 out of 105 , which is usual the cut-off for the diagnosis of global cognitive deterioration compatible with Alzheimer-type dementia [66]. A decline or improvement in a given cognitive domain was defined as a decrease or increase of $10 \%$ relative to the baseline score. For this last analysis, raw scores were used instead of the standardized scores. Decline or improvement on the CAMCOG was defined as a decrease or increase respectively of 10 points compared with the baseline score.

The SPSS version 10 package was used for statistical analysis. Statistical analysis was conducted using descriptive statistics to define the number of patients with and without a disorder at 1 and at 6 months post-stroke. Patients with cognitive disorders were compared with patients without cognitive disorders using Student's two-tailed $\mathrm{t}$ test for continuous variables and $\chi^{2}$ analysis for categorical variables. 


\section{Results}

The number of patients with and without a disorder of a given domain at 1 and 6 months after stroke are listed in Table 1. For all cognitive domains, except for simple speed most patients scored above the cut-off at 1 month after stroke and remained at this level at 6 months (Overall cognitive functioning, N=96; Memory, N=90; Cognitive flexibility, $\mathrm{N}=53$ ). There were some patients who scored below the cut-off at 1 month after stroke but who scored above the cutoff either on memory $(\mathrm{N}=13)$, cognitive flexibility $(\mathrm{N}=15)$ or overall cognitive functioning $(\mathrm{N}=16)$ or on a combination of these cognitive domains at 6 months after stroke. A small subsample of the population deteriorated to a score below the cut-off at 6 months after stroke.

\begin{tabular}{|c|c|c|c|c|c|c|c|}
\hline \multicolumn{2}{|l|}{ Cognitive } & \multicolumn{3}{|c|}{1 month } & \multicolumn{3}{|c|}{6 months } \\
\hline Functioning & $\mathrm{N}$ & & & & $\mathrm{AB}$ & Unchanged & $\mathrm{BE}$ \\
\hline \multirow{2}{*}{$\begin{array}{l}\text { Overall } \\
\text { (CAMCOG) }\end{array}$} & \multirow[t]{2}{*}{139} & 100 & Above & Cut-off & & 96 & 4 \\
\hline & & 39 & Below & Cut-off & 16 & 23 & \\
\hline \multirow[t]{2}{*}{ Memory } & \multirow[t]{2}{*}{117} & 91 & Above & Cut-off & & 90 & 1 \\
\hline & & 26 & Below & Cut-off & 13 & 13 & \\
\hline \multirow[t]{2}{*}{ Simple Speed } & \multirow[t]{2}{*}{115} & 58 & Above & Cut-off & & 51 & 7 \\
\hline & & 57 & Below & Cut-off & 12 & 45 & \\
\hline \multirow{2}{*}{$\begin{array}{l}\text { Cognitive flex- } \\
\text { ibility }\end{array}$} & \multirow[t]{2}{*}{107} & 58 & Above & Cut-off & & 53 & 5 \\
\hline & & 49 & Below & Cut-off & 15 & 34 & \\
\hline
\end{tabular}

AB: Above Cut-off | BE: Below Cut-off

As scoring above or below a cut-off gives no information about the degree of improvement or deterioration, we also analysed the data in terms of improvement or deterioration in comparison with the first assessment. These analyses were independent of whether patients scored above or below the cut-off. Of overall cognitive functioning $12.9 \%$ of the patients scored at least 10 points higher than their baseline-score, $83.5 \%$ had the same score, and 3.6\% deteriorated at least 10 points compared with their first score. Of memory $52.1 \%$ improved at least $10 \%$ compared to their baseline score, $37.6 \%$ stayed stable and $10.3 \%$ deteriorated. Of simple speed and cognitive flexibility, $37.2 \%$ and $41.8 \%$ respectively, improved, $49.6 \%$ and $44.6 \%$ respectively, stayed stable, and $12.4 \%$ and $13.6 \%$ respectively, deteriorated compared with their baseline-scores.

At 1 month after stroke, more women than men had a memory disorder $(p=.039)$. Patients with simple speed disorders and disturbed ratings on the 
CAMCOG at 1 month after stroke were more likely to be older $(t=3.2, d f=113$, $\mathrm{p}=.01$ and $\mathrm{t}=3.0, \mathrm{df}=137, \mathrm{p}=.01$, respectively), have a lower education

$\left(\chi^{2}=9.5, \mathrm{df}=2, \mathrm{p}=.01\right.$ and $\chi^{2}=10.4, \mathrm{df}=2, \mathrm{p}=.01$, respectively $)$, and be of female $\operatorname{sex}\left(\chi^{2}=4.7, \mathrm{df}=1, \mathrm{p}=.03\right.$ and $\chi^{2}=5.5, \mathrm{df}=1, \mathrm{p}=.02$, respectively). There were no differences between patients on tests of cognitive flexibility (see Table 2a). The same pattern was seen at 6 months, except that patients with memory disorders were older $(t=2.3, d f=115, p=.03)$. Men and women had a similar overall cognitive function and memory function $\left(\chi^{2}=2.1, \mathrm{df}=1, \mathrm{p}=.15\right.$ and $\chi^{2}=$ $1.9, \mathrm{df}=1, \mathrm{p}=.17$ respectively) (see Table $2 \mathrm{~b}$ ).

Table 2: Difference between patients with and without cognitive disorders

\begin{tabular}{|c|c|c|c|c|c|c|c|c|}
\hline \multirow[b]{2}{*}{1 Month } & \multicolumn{2}{|c|}{ Memory } & \multicolumn{2}{|c|}{ Simple Speed } & \multicolumn{2}{|c|}{ Flexibility } & \multicolumn{2}{|c|}{ Overall } \\
\hline & Below & Above & Below & Above & Below & Above & Below & Above \\
\hline Age (mean) & 70.4 & 67.7 & 72.2 & $65.0^{*}$ & 69.4 & 66.0 & 74.3 & $67.4^{*}$ \\
\hline $\begin{array}{l}\text { Education } \\
(\mathrm{L} / \mathrm{H})\end{array}$ & $15 / 11$ & $43 / 48$ & $36 / 21$ & $20 / 38^{*}$ & $24 / 25$ & $25 / 33$ & $27 / 12$ & $45 / 55^{*}$ \\
\hline $\operatorname{Sex}(M / F)$ & $11 / 15$ & $59 / 32^{*}$ & $29 / 28$ & $41 / 17^{*}$ & $29 / 20$ & $38 / 20$ & $16 / 23$ & $63 / 37^{*}$ \\
\hline \multicolumn{9}{|l|}{6 Months } \\
\hline Age (mean) & 75.3 & $67.4^{*}$ & 72.8 & $65.1^{*}$ & 69.5 & 66.4 & 76.6 & $67.6^{*}$ \\
\hline $\begin{array}{l}\text { Education } \\
(\mathrm{L} / \mathrm{H})\end{array}$ & $10 / 4$ & $48 / 55$ & $33 / 19$ & $23 / 40^{*}$ & $22 / 17$ & $27 / 41$ & $19 / 8$ & $53 / 59^{*}$ \\
\hline $\operatorname{Sex}(M / F)$ & $6 / 8$ & $64 / 39$ & $30 / 22$ & $40 / 23^{*}$ & $23 / 16$ & $44 / 24$ & $12 / 15$ & $67 / 45$ \\
\hline
\end{tabular}

Below: below cut-off | Above: Above cut-off $\mid \mathrm{L}=$ low $\mid \mathrm{H}=$ high $\mid \mathrm{M}=$ male |

$\mathrm{F}=$ female $\mid{ }^{*} \mathrm{p}<.05$

\section{Discussion}

We examined a consecutive series of stroke patients, at 1 and 6 months after stroke. Unlike most studies, the first assessment occurred early, within 1 month after stroke. This approach made it possible to determine the course of development of cognitive disorders. On all cognitive domains, except for simple speed, most patients who scored below the cut-off at 1 month after stroke scored above the cut-off at 6 months after stroke. By analysing the data in terms of improvement or deterioration compared to the baseline scores we found that a substantial group of the patients improved on at least one cognitive domain at 6 months after stroke. Most patients remained stable; in only a small number of patients did cognitive functioning deteriorate between 1 and 6 months after stroke. It cannot be precluded that a practice effect was involved, but this is unlikely 
because the changes were selective and not for all cognitive domains.

Obviously, most cognitive functions improve spontaneously after stroke, probably as a consequence of adaptation in the brain. A process of spontaneous brainrepair might have occurred between the two measurements. However, this improvement has not been found in patients who had simple speed disorders in whom subcortical or white matter lesions on CT or MRI scan are prevalent [67-70]. Moreover, because several brain structures are probably involved in the performance of speed tasks, it is unlikely that a cerebral adaptation occurs. Cognitive disorders in the other domains may be explained by a more focal type of damage.

In contrast to these findings, others have reported, for a cohort of 25 stroke patients, an improvement on time-limited tasks and concluded that this cognitive domain was susceptible to subcortical pathology [56]. The discrepancy between their findings and ours can be explained by the use of different measures of speed: Bowler et al. studied what would be called complex speed in our study, whereas we measured simple speed. Prevo et al. [61] found an improvement on specific cognitive domains like memory, neglect and visual-spatial disorders. Others also reported an improvement on memory tasks at 6 months after stroke compared with memory performance at 3 months [57]. On the basis of our study, one would expect an even greater improvement than that seen in that study because we tested patients at 1 month after stroke rather than at 3 months. Ebrahim [58] found that cognitive impairment is common after stroke and affects more than $10 \%$ of patients who survived at 6 months. They also found that $45.5 \%$ of the patients showed cognitive deterioration between 1 and 6 months after stroke. This contrasts with our study, which showed an improvement on overall cognitive functioning. These differences may be related to different tests used. Female sex and older age were identified as risk factors for developing cognitive disorders, which is in line with other studies [32, 71, 72]. A strength of our study is that, by including only patients with a first-ever cerebral stroke, we had a more homogeneous group of patients, which makes it possible to extrapolate our findings to a clinical population of patients with first-ever stroke.

However, our study had some methodological limitations. First, we used composite scores, based on theoretical concepts, as indicators of different cognitive domains. It is possible that these composite score are not independent of each other. However, we believe that the theoretically based composite scores we formed are clinically more relevant than those based on statistical analyses. Second, there was selective attrition on some cognitive domains. Patients who could not complete the whole test battery were probably more cognitively impaired than those who completed all the tests. This might have led to underestimation of the number of patients with cognitive disorders in our study. Overall, we conclude that in general the prognosis of cognitive functioning after 
stroke is positive. However, more extensive neuropsychological testing over a longer follow-up period is needed to define exactly which cognitive domains are affected or improve and to identify risk factors or protective factors. 


\section{Cognitive functioning after stroke: a one-year follow-up study}

Adapted from:

Rasquin SMC, Lodder J, Ponds RWHM, Winkens I, Jolles J, Verhey FRJ. Cognitive functioning after stroke: a one-year follow-up study. Dementia and Geriatric Cognitive Disorders. 2004;18:138-144. 


\section{Abstract}

Background: Cognitive disorders after stroke are one of the main causes of disability in daily activities. The main aim of this study was to investigate the frequency of post-stroke dementia, post-stroke Mild Cognitive Impairment (MCI), and post-stroke amnestic MCI at different times after first-ever stroke in 196 patients. In addition, cognitive disorders and their course were studied.

Results: Frequency of post-stroke dementia was about $10 \%$ at all the evaluation times. Most patients had post-stroke MCI. Of the cognitive functions investigated, mental speed and calculation were the most frequently disturbed. Performance on almost all cognitive tests was improved 6 and 12 months after stroke.

Conclusions: While the frequency of post-stroke dementia is low, the frequency of post-stroke MCI is high but improvement of cognitive function is possible. 


\section{Introduction}

Stroke can have dramatic consequences for patients and their environment. Besides physical handicaps, cognitive disorders can also contribute to disability in every-day life [73]. Most studies of cognitive disorders after stroke report Vascular Dementia (VaD) as the main outcome $[16,62,63], 8$ to $26 \%$ of stroke patients develop a dementia syndrome within 12 months [2, 74, 75]. The variation in prevalence rates is attributable to different patient populations and to differences in diagnostic criteria [4]. One of the problems with the current criteria (DSM IV, NINDS-AIREN, ADDTC, and ICD-10) for VaD is that they are largely based on the definition of Alzheimer type dementia. For instance, in all criteria, except the ADDTC, a memory disorder is required of the diagnosis of dementia, however, stroke may primarily affect other cognitive functions [76]. For example, stroke affects the performance on tasks involving mental speed [56, 77], even more than memory performance [29]. Thus when studying the cognitive consequences of stroke, it is important to investigate a broad range of cognitive functions and not solely dementia.

To emphasize this broader approach, Bowler and Hachinski introduced the concept Vascular Cognitive Impairment (VCI) [7]. VCI describes a whole range of cognitive impairments from the lowest level of cognitive impairment to dementia caused by vascular brain damage. Few data exist about the prevalence of the milder forms of cognitive impairment, also called vascular Cognitive Impairment No Dementia (CIND) or vascular Mild Cognitive Impairment (MCI) $[10,15,78-80]$. The concept MCI originally focused on memory disorders whereas CIND is not limited to one cognitive domain.

The purpose of this longitudinal study was to investigate the prevalence of dementia and other cognitive disorders at different times after stroke and changes in cognitive functioning with time.

\section{Methods}

\section{Patients}

Participants of this study took part in a large study called CODAS (COgnitive Disorders After Stroke). This is a longitudinal, prospective clinical study aimed at investigating cognitive functioning, risk factors for cognitive impairment, and the course of cognitive functioning after stroke [29]. Patients with a first-ever cerebral stroke (not located in the brainstem or cerebellum) were assessed clinically and neuropsychologically at 1, 6, 12, and 24 months after stroke. For this 
report the data of the first three measurements were available.

Patients who were admitted to the hospital or who visited the outpatient clinic of the Academic Hospital Maastricht between January 2000 and August 2001 because of a stroke were asked to participate in this study. An experienced neurologist diagnosed stroke based on clinical findings and data supported by a Computed Tomography (CT)-scan evaluated by means of standardized criteria [53]. The following CT features were recorded; whether the stroke was ischemic or haemorrhagic, side of the stroke, stroke-type (territorial or lacunar), presence of white matter lesions, silent infarcts, and atrophy.

Inclusion criteria were: first ever cerebral stroke, age older than 40 years, fluency in Dutch, no other neurological or psychiatric disorders, and a Mini Mental State Examination (MMSE) score $\geq 15$ (as such patients with severe cortical deficits were excluded, and a group of patients remain, who could be tested reliably). Hundred and ninety-six patients were included (mean age 68.5 years; 107 men and 89 women). Cognitive functioning was compared with that of a norm group from the Maastricht Aging Study (MAAS), a study of older healthy volunteers from the region south Limburg investigating features which predispose to normal cognitive aging. Norm tables are stratified according to age, sex, and level of education [49]. In the MAAS-project healthy subjects were recruited from the Registration Network General Practioners. The population is representative for the Limburg and Dutch populations with respect to demographic characteristics (age, gender, educational level and type of health care) [49].

\section{Diagnosis post-stroke Cognitive Disorders}

Post-stroke Dementia: Dementia was diagnosed independently by an experienced neuropsychiatrist and a neuropsychologist according to the DSM IV criteria. Both clinicians are experts in the field of dementia. The diagnosis was based on all available data, including information about medical history, structured interview with an informant, clinical observation, and test performances. Interference with daily activities due to cognitive deficits was assessed by the Interview for Deterioration in Daily life in Dementia (IDDD) [38]. The diagnosis of Vascular dementia was based on the NINDS-AIREN criteria [24]. When there was a disagreement between the two clinicians, the final diagnosis was negative; this was the case in 5 patients. Agreement between the two clinicians was excellent $(\mathrm{K}=0.88 ; \mathrm{p}=.00)$.

In order to determine whether dementia was present before the stroke, a proxy of the patient was asked whether the patient met in retrospect each of the criteria for dementia of the DSM IV (whether any signs of memory disorder, aphasia, apraxia, agnosia or executive dysfunctioning existed before the stroke; and whether these cognitive impairments interfered with daily activities). 
Post-Stroke MCI: Post-stroke MCI was diagnosed if patients fulfilled the following criteria: cognitive disorder on at least one of the following cognitive functions: memory, language, orientation, mental speed, attention, praxis, executive functioning, calculation and visuospatial functioning; no interference of the cognitive disorders with daily activities, assessed by the IDDD; and no dementia. These criteria are largely based on the criteria of MCI [81].

Post-Stroke amnestic MCI: Patients with the following features were classified as post-stroke amnestic MCI: only a memory disorder; the memory disorder did not interfere with daily activities, assessed by the IDDD; and no dementia. These criteria are based on the recent adapted criteria for MCI [81].

\section{Assessment of neuropsychological functioning}

CAMCOG: This is the cognitive part of the Cambridge Examination for Mental Disorders of the Elderly (CAMDEX), a standardized instrument for the diagnosis and gradation of dementia in the elderly. The CAMCOG can be subdivided into 10 subscales: orientation, language-expression, language-understanding, memory (long and short term), attention, calculation, praxis, abstract thinking, and abstract perception. The Dutch version of the CAMCOG was used [39], which has been shown to be well tolerated and sensitive for the detection of cognitive disorders in stroke patients [40].

Auditory Verbal Learning Test (AVLT): This test is used to measure episodic memory. The variable of interest is the number of words recalled after each trial and after a 20-minute delay [42]. To minimise the influence of a learning effect, we used parallel versions of the AVLT.

Concept Shifting Task (CST): This test is derived from the Trail Making Test and is used to measure the ease of shifting between two concepts. The variable of interest is the time needed to complete each of the four subtests [82].

Stroop colour word test: The Stroop test is a test of selective attention and interference susceptibility. The variable of interest is the time needed to complete each of the three subtests [45].

Calculation: This test is part of the Groninger Intelligence Test (GIT) and involves the correct completion of as many sums as possible in 1 minute. It measures arithmetic skills [47].

Mental Rotation: This mental rotation test is also part of the GIT. It requires the subject to indicate which two-dimensional shapes from a larger set are needed to exactly fill up a given space on the test page. This test measures visuospatial abilities [47]. Normative data were available for all psychometric tests used in this study, except for the CAMCOG. 


\section{Cognitive domains}

Nine cognitive domains were formed based on the neuropsychological test battery: memory, mental speed, executive functioning, orientation, attention, language, praxis, visuospatial abilities, and calculation. These cognitive domains (except for mental speed) are based on the cognitive domains mentioned in the NINDS-AIREN criteria for $\mathrm{VaD}$.

Three cognitive functions were selected based on data in the literature: memory, mental speed, and executive functioning [83]. Compound scores were derived for these three cognitive functions. Patient scores were converted to standardized scores with the following formula: $Z=$ (patient score - norm score)/ standard deviation from the norm group. Memory was calculated from the mean of the Z- scores of the total score after five trails and the recall score after 20 minutes. For mental speed the mean of the Z-scores of the CST I/II/0 and STROOP I was used. The mean of the Z-interference-scores from the Stroop and CST was used to define the cognitive domain 'executive functioning'. The interference scores of the Stroop and CST were defined by the following formula: Part III - mean (Part I and part II).

A score lower than the 10th percentile (a cut-off commonly used in clinical practice) compared to the norm group defined a cognitive disorder. To define improvement or deterioration on cognitive tests, the Reliable Change Index (RCI) was used [84]. The RCI can be used to determine if a change observed in a subject is clinically significant. The amount of change between two measurement times is divided by the standard measurement error for the score of difference (this can be conceived as the random error in sample survey)[85]. A score exceeding the range of -1.96 to 1.96 reflects a significant change [84]. For this analysis, cognitive test scores were used, except for the CAMCOG (no norms were available).

\section{Statistical Analysis}

Statistical analyses were performed with the statistical package for Social Sciences version 10 (SPSS-10). Descriptive statistics were used to define the number of patients with one of the cognitive disorders or cognitive impairments. The number of patients that improved or deteriorated was also analyzed with descriptive statistics. Differences between groups at baseline with regard to CT-variables were performed with $\chi^{2}$. To perform valid analyses with sufficient power, we imputed data for some patients with missing data according to the method described in Tabachnick et al [51], in which an experienced clinician decided, based on available data and the patients' neuropsychological profile, how to impute the data. Data imputation was performed if there was at least one 
other test available for the same cognitive domain and if the missing test was administered at an earlier or later measurement time. For instance, in $22.4 \%$ and $23.4 \%$ of the cases there were at baseline some data missing of the CST I-III and Stroop I-III respectively, in $28.9 \%$ and $16.9 \%$ of whom imputation took place, and in $12.8 \%$ of the patients data with regard to the AVLT were missing, and $8.0 \%$ were imputed. The rate of missing data at the 6 months assessment was somewhat lower (12.2\% for the CSTI-III, and $17.4 \%$ for the Stroop I-III) whereas imputation was done in $9.5 \%$ and $16.7 \%$ respectively. At 12 months after stroke data were comparable to imputation at 6 months after stroke.

\section{Results}

During the inclusion period, 592 patients came to the hospital with a stroke. Of these 592 patients 80 died within 1 month after stroke and another 316 were excluded ( 89 were not first-ever strokes, 57 had a stroke located in the brainstem or cerebellum, 46 MMSE $<15,34$ had severe aphasia, 20 had comorbid neurological or psychiatric disorders, 6 were in a coma, 5 were not native Dutch speaking, 9 were younger than 40 years, 9 lived too far from the hospital, 6 were admitted too long after their stroke and 35 refused to participate), hundred and ninety-six were eligible for the CODAS study. Baseline demographic variables, MMSE-scores and stroke-related data of the patients are presented in table 1.

\begin{tabular}{ll}
\hline Table 1: Patient characteristics at baseline \\
\hline $\mathrm{N}$ & 196 \\
Education low/high & $110 / 86$ \\
Sex M/F & $107 / 89$ \\
Age mean (SD) & $68.4(12.5)$ \\
MMSE 1 mean (SD) & $25.4(3.6)$ \\
MMSE 2 mean (SD) & $25.1(5.5)$ \\
MMSE 3 mean (SD) & $25.2(5.6)$ \\
Territorial-Lacunar-Heamorrhagic (N = 194) & $39.2 \%-52.6 \%-8.2 \%$ \\
Side: left-right-both $(\mathrm{N}=190)$ & $41.6 \%-56.8 \%-1.6 \%$ \\
White matter lesions present $(\mathrm{N}=181)$ & $24.9 \%$ \\
Silent infarcts present $(\mathrm{N}=179)$ & $35.2 \%$ \\
Atrophy present $(\mathrm{N}=187)$ & $70.1 \%$ \\
\hline SD: standard deviation | MMSE 1,2,3: 1, 6, and 12 months
\end{tabular}


Six months after stroke, 172 patients $(87.4 \%)$ could be tested, and at 12 months 159 patients (80.4\%). Main reasons for drop-out were refusal or death. Patients who dropped out did not differ from patients who remained in the study in terms of baseline MMSE ( 6 months: $\mathrm{t}=0.29, \mathrm{p}=.78 ; 12$ months: $\mathrm{t}=-1.59, \mathrm{p}=.11$ ), age (6 months: $\mathrm{t}=1.1, \mathrm{p}=.26 ; 12$ months: $\mathrm{t}=1.1, \mathrm{p}=.27)$, education ( 6 months: $\chi^{2}=2.4, \mathrm{p}=.17 ; 12$ months: $\left.\chi^{2}=1.4, \mathrm{p}=.23\right)$, or $\operatorname{sex}\left(6\right.$ months: $\chi^{2}=2.9, \mathrm{p}=.08 ; 12$ months: $\chi^{2}=0.09, \mathrm{p}=.77$ ).

Table 2 describes the prevalence rates of post-stroke dementia, post-stroke $\mathrm{MCI}$, and post-stroke amnestic MCI.

Table 2: Number of patients and Prevalence rates (percentages) of VaD, MCI, amnestic MCI

\begin{tabular}{lllllll}
\hline & \multicolumn{2}{l}{ 1 month } & \multicolumn{2}{l}{ 6 month } & \multicolumn{2}{l}{12 month } \\
\hline No cognitive disorders & 34 & $17.5 \%$ & 36 & $18.6 \%$ & 40 & $20.6 \%$ \\
VaD & 21 & $10.8 \%$ & 15 & $7.7 \%$ & 15 & $7.7 \%$ \\
Post-stroke MCI & 138 & $71.1 \%$ & 119 & $61.3 \%$ & 100 & $51.5 \%$ \\
Post-stroke amnestic MCI & 1 & $0.5 \%$ & 0 & $0.0 \%$ & 2 & $1.0 \%$ \\
\hline
\end{tabular}

Two patients appeared to be demented before stroke. These patients were excluded from the analyses because we were interested in the prevalence of dementia after stroke. Twenty-four patients (12.2\%) developed dementia within 1 year after stroke. Of these 15 (62.5\%) had both evidence of CVD on CT scan and developed dementia within 3 months after stroke, thus fulfilling the criteria of probable $\mathrm{VaD}$. The other 9 patients were diagnosed as possible $\mathrm{VaD}$ because of negative scan in 6 patients (25.0\%) and developing dementia after 3 months in 3 patients $(12.5 \%)$. Seven patients were demented at baseline, but were no longer considered to be demented later on in the study. Only three patients who were not demented at 1 or 6 months became demented later on. Most patients were classified as having post-stroke $\mathrm{MCI}$, whereas very few $(0.5 \%, 0 \%$ and $1.0 \%$ for each measurement time) were classified as having post-stroke amnestic MCI. During the one-year follow-up 30 patients deteriorated (to a more severe cognitive deficit) compared to an earlier measurement period. Patients who deteriorated were older $(\mathrm{t}=2.7, \mathrm{p}=.01)$ and had lower baseline MMSE-scores $(\mathrm{t}=$ $-2.6, p=.01$ ) compared to patient without a deterioration, whereas there was no difference in stroke-related variables, education and sex.

Patients with dementia at baseline had more often territorial infarcts, and more often white matter lesions, silent infarcts, and atrophy on CT scan, compared to 
patients with post-stroke $\mathrm{MCI}$ and patient without cognitive disorders, but these differences were not significant. Patients with dementia had significantly more often a stroke in the left hemisphere compared to patients without any cognitive disorder, while patients with post-stroke MCI were intermediate $(52.4 \%, 32.2 \%$ and $40.9 \%$ respectively). Because of the low number of patients with amnestic $\mathrm{MCI}$, this group was left out for these analyses.

Table 3 presents the number of patients with impairment of one or more cognitive functions at each measurement time. Mental speed and calculation were the cognitive functions that were the most often disturbed.

\begin{tabular}{llllllll}
\hline $\begin{array}{l}\text { Table 3: Cognitive functioning at 1, } 6 \text { and } 12 \\
\text { or without (-) cognitive impairments }\end{array}$ \\
\hline \multicolumn{7}{c}{ 1 month } & \multicolumn{6}{l}{6 month } & \multicolumn{3}{l}{12 month } \\
\hline CAMCOG total & - & + & - & + & - & + \\
Memory & 55.7 & 44.3 & 66.5 & 33.5 & 66.2 & 33.8 \\
Speed & 75.6 & 24.4 & 83.4 & 16.6 & 84.6 & 15.4 \\
Executive & 39.8 & 60.2 & 46.4 & 53.6 & 50.6 & 49.4 \\
Orientation & 67.9 & 32.1 & 75.7 & 24.3 & 77.8 & 22.2 \\
Attention & 89.7 & 10.3 & 92.9 & 7.1 & 91.7 & 8.3 \\
Language & 68.0 & 32.0 & 65.3 & 34.7 & 69.4 & 30.6 \\
Praxis & 64.9 & 35.1 & 71.8 & 28.2 & 74.5 & 25.5 \\
Visuospatial & 68.6 & 31.4 & 77.1 & 22.9 & 79.6 & 20.4 \\
Calculation & 63.5 & 36.5 & 77.2 & 22.8 & 70.4 & 29.6 \\
\hline & 47.9 & 52.1 & 57.4 & 42.6 & 62.6 & 37.4 \\
\hline
\end{tabular}

Table 4 shows the number of patients whose performance on the cognitive tests improved, deteriorated, or did not change. In the period 1 to 6 months, more people showed performance improvement than performance deterioration. This was also the case for the period 6 to 12 months, except for the CST I/III, calculation, and mental rotation tasks, in which more patients showed performance deterioration than performance improvement. In both comparisons (6 versus 1 month and 12 versus 6 months), the performance of most patients did not change. 


\begin{tabular}{|c|c|c|c|c|c|c|}
\hline \multirow[b]{3}{*}{ Test } & \multirow{2}{*}{\multicolumn{3}{|c|}{$\underline{6 m \text { versus } 1 \mathrm{~m}}$}} & \multirow{2}{*}{\multicolumn{3}{|c|}{$\underline{12 \mathrm{~m} \text { versus } 6 \mathrm{~m}}$}} \\
\hline & & & & & & \\
\hline & imp & sam & det & imp & sam & det \\
\hline CST I & 31.6 & 57.8 & 10.6 & 15.7 & 67.9 & 16.4 \\
\hline CST II & 25.7 & 62.2 & 12.1 & 22.0 & 62.8 & 15.2 \\
\hline CST III & 33.3 & 55.8 & 10.9 & 17.9 & 56.1 & 26.0 \\
\hline Stroop 1 & 16.7 & 74.6 & 8.7 & 9.5 & 82.6 & 7.9 \\
\hline Stroop 2 & 23.5 & 69.9 & 6.6 & 19.8 & 73.1 & 7.1 \\
\hline Stroop 3 & 26.7 & 61.8 & 11.5 & 19.3 & 70.6 & 10.1 \\
\hline Total $\mathrm{N}$ of words & 18.8 & 76.3 & 4.9 & 11.1 & 82.2 & 6.7 \\
\hline Recall & 23.6 & 67.1 & 9.3 & 12.2 & 77.9 & 9.9 \\
\hline Caculation & 10.6 & 84.7 & 4.7 & 5.9 & 86.3 & 7.8 \\
\hline Mental Rotation & 4.7 & 91.8 & 3.5 & 1.3 & 92.8 & 5.9 \\
\hline
\end{tabular}

\section{Discussion}

Few patients would appear to become demented after stroke (12\%), although other studies have reported higher rates of dementia prevalence $(18 \%-26 \%)$ 3 months after stroke $[2,6,17]$. The lower prevalence rate in our study could be because we excluded people who had had a second stroke or who had an MMSE < $15(7.7 \%$ patients $(\mathrm{N}=46))$. The other studies included patients with severe cognitive deficits, other than aphasia $[2,6,17]$. If we had not excluded the patients with severe cognitive deficits at baseline and if we assume that these patients would have been demented, the proportion of patients with post-stroke dementia would be $27.8 \%$ which is similar to that of other studies $[2,6,17]$. In our sample, the percentage of patients with prestroke dementia is low compared to others who found a prevalence of $16.3 \%$ of prestroke dementia [86,87]. This is probably also related to the exclusion of patients with a MMSE $<15$.

Most research into cognitive disorders after stroke is focused on the development of dementia, but other, less severe cognitive disorders could also be present. These other cognitive disorders may be mild in an early stage but may carry the risk of dementia in the long term, and as such are clinically relevant to investigate. 'Mild Cognitive Impairment' (MCI), is the term often used to describe this transitional phase and is mostly used in the development of Alzheimer's disease. The traditional term MCI, which focused on memory disorders only, has been replaced by other concepts such as 'amnestic MCI' [81]. We found only 2 patients with post-stroke amnestic MCI, and thus it is doubt- 
ful whether this concept is applicable for a pre-dementia stage after stroke. The traditional concept $\mathrm{MCI}$ has been further adapted to include disturbances of cognitive functions other than memory [81] and this new concept would appear more appropriate to describe the transitional phase into dementia development, especially in post-stroke dementia. In our study almost $65 \%$ of the patients had multiple cognitive deficits, but not as severe to warrant a diagnosis of dementia. Thus cognitive deficits after stroke (including subtle deficits) are frequent, and in fact more frequent than may be thought in daily practice. As these cognitive deficits may cause to some extent impairments in daily activities, this figure may point towards a problem hidden so far [73].

The patients investigated had relatively few memory disturbances. Mental speed and calculation were the most frequently disturbed cognitive functions, as was found by other researchers $[18,56,88]$. These results suggest that, in investigations of the early stage of $\mathrm{VaD}$ the emphasis should not be solely on memory but should include other cognitive disorders.

The prognosis of dementia in our study was not always unfavorable; one third of the patients who were demented at baseline were no longer considered to be demented later in the study, mostly (5 out of the 7) because of the fact that they did not fulfil anymore the criterion of interference of daily life activities. Also on most neuropsychological tests patients improved or did not change. This has consequences for the way patients are informed directly after stroke. The prognosis regarding the development of cognitive disorders after stroke is in general favorable and recovery is possible. Desmond also found improvement in 12.5\% of the stroke patients 1 year after stroke [89]. Schmidt found that $24 \%$ of patients with a first-ever cerebral stroke had an improved performance 6 months after stroke [90]. Ballard found that even $50 \%$ of the patients improve 15 months after stroke (improvement defined as 2 points increase on the MMSE) [91]. The influence of treatment such as, cognitive rehabilitation, blood pressure regulation, or cholesterol regulation on improvement is not known. However it should be remembered that the condition of some patients deteriorated or that they developed cognitive disorders and did not show improvement, which could be the consequence of increasing brain damage due to silent infarcts [92]. Patients who are older and had a lower baseline MMSE were at greater risk of deterioration. Stroke-related factors were not related to decline.

A shortcoming of our study is that the diagnosis of dementia was not based on direct clinical contact with the patient. However all data necessary for the diagnosis dementia were available, and information was collected in a structured and highly standardized approach, including informant's reports on daily functioning, clinical observation by trained research psychologists, and neuropsychological testing. A second point to address is that patients were tested three times, which could have led to a learning effect. Although this effect could not be ruled out, this effect is minimal for the Stroop after the second administration 
and this also holds for the CST, and moreover in the last part of the CST there seems to be no learning effect. Also the AVLT parallel versions do not have a learning effect [93]. An advantage of our study is that we had a representative patient population, in which the patients were tested neuropsychologically directly after stroke, for the first time at 1 month. Most studies investigate cognitive performance directly after stroke, mostly 3 months after stroke [2, 18, 32]. If cognitive performance is tested later after stroke, damage could be too severe, so as to render treatment ineffective.

In conclusion, few patients become demented after stroke, and most cognitive disturbances are in the field of mental speed and calculation. While improvement is possible, in a small group the cognitive disturbances do not improve or even deteriorate. Future research features should investigate aspects that influence the course of cognitive disorders after stroke. Research that is not restricted to dementia after stroke but which encompasses the whole range of cognitive disorders can serve as a starting point for treatment and rehabilitation. 


\section{The effect of different diagnostic criteria on the prevalence and incidence of post-stroke dementia}

Adapted from:

Rasquin SMC, Verhey FRJ, Lodder J. The effect of different diagnostic criteria on the prevalence and incidence of post-stroke dementia. Neuroepidemiology. In press. 


\section{Abstract}

Background: There is little agreement about the prevalence and incidence of $\mathrm{VaD}$, mainly because investigators have used different diagnostic criteria. The aim of this study was to examine the influence of different diagnostic criteria on the prevalence and cumulative incidence of $\mathrm{VaD}$.

Methods: First-ever stroke patients ( $\mathrm{N}=194)$, were clinically evaluated at 1, 6, 12, and 24 months after stroke. Post-Stroke VaD was diagnosed using the DSM-III, DSM-III-R, DSM-IV, ICD-10-NA, NINDS-AIREN, and ADDTC criteria.

Results: The prevalence of $\mathrm{VaD}$ was highest at 1 month; ranging from $11.3 \%$ with the NINDS-AIREN to $20.1 \%$ with the ICD-10-NA. The incidence was highest at 6 months, ranging from 2.6\% with the ADDTC to $5.2 \%$ with the ICD-10NA. Agreement among diagnostic criteria was high, with the exception of the ADDTC.

Conclusions: Both the prevalence and incidence of dementia are highest directly after stroke but exact rates are influenced by the diagnostic criteria used. 


\section{Introduction}

Vascular dementia (VaD) is, after Alzheimer's disease (AD), the second main cause of dementia in Western countries, with a prevalence varying from $8 \%$ to $26 \%$, depending on the diagnostic criteria used [2-5]. In the last 20 years nine sets of criteria for VaD have been proposed. In 1974, Hachinski et al. introduced the term 'multi infarct dementia' (MID) [20]. While this term suggests that only multiple infarcts can lead to dementia, this is not the case because also other vascular mechanisms can cause VaD. The Diagnostic and Statistical Manual of Mental Disorders (DSM) and International Classification of Disease 10th version, neurological adaptation (ICD-10-NA), formulated the underlying mechanisms more clearly [23, 94-96]. However, these criteria do not specify etiological mechanisms for $\mathrm{VaD}$. These shortcomings have led to the formulation of two consensus-based criteria: the National Institute for Neurological Diseases and Syndromes - Association Internationale pour la Recherche et l'Enseingement en Neurosciences (NINDS-AIREN), Alzheimer's Disease Diagnostic and Treatment Centres (ADDTC) [24, 25]. These criteria state more clearly the relationship between dementia and the underlying pathology, in terms of a temporal relationship between cerebrovascular damage (CVD) and dementia, and require the inclusion of a neuroradiological examination.

In contrast to the variation in the prevalence of $\mathrm{VaD}$ between studies, the incidence of $\mathrm{VaD}$ is more consistent [97]. In a study by Tatemichi et al. [98], 5.4\% of the patients with ischemic stroke, who were initially free of dementia became demented within 1 year. Two other studies reported an incidence of $\mathrm{VaD}$, diagnosed with the DSM-III-R of 8.4 and 8.5 per 100 person-years in a stroke population whereas this rate was much lower (1.3 per 100 person-years) in the control group [32]. In a study of the cumulative incidence of $\mathrm{VaD}$ (defined by the DSM-III-R), 7\% of stroke patients were demented 3 months after stroke, and this increased to $29 \%$ after 1 year, and to $34 \%$ in the subsequent 2 years [99]. Henon et al. [86] found in a longitudinal study (3 years post-stroke) an incidence of $\mathrm{VaD}$, defined according to the NINDS-AIREN criteria of 12.3 per 100 personyears. Until now, little is known about the influence of different diagnostic systems on the cumulative incidence of $\mathrm{VaD}$.

The aim of this study was to measure the prevalence and cumulative incidence of $\mathrm{VaD}$ diagnosed according to the DSM-III, DSM-III-R, DSM-IV, NINDSAIREN, ADDTC, and ICD-10-NA criteria in a cohort of patients who had had a stroke. 


\section{Methods}

\section{Patients}

Patients were participants of the CODAS study, a prospective study (COgnitive Disorders After Stroke), which has been described earlier [29, 100, 101]. Patients included in the study had had a first-ever hemispheric stroke, were older than 40 years, had adequate post-stroke fluency in Dutch, and had an initial Mini Mental State Examination (MMSE) score $\geq 15$ (to ensure neuropsychological testing is possible). Severe aphasia, pre-stroke dementia, and other neurological or major psychiatric disorders were exclusion criteria.

\section{Procedure}

Patients were asked within 48 hours of stroke to participate in the study. Participants were administered a clinical and neuropsychological examination at 1, 6, 12, and 24 months post stroke. The assessment at one month served as the baseline measure in the present study. Cognitive functioning was compared with that of a norm group from the Maastricht Aging Study (MAAS); norm tables were stratified according to age, sex, and level of education [49]. Cognitive impairment was defined as a score below the 10th percentile of the z-scores of the norm group from MAAS (equal to a standard deviation of 1.28); this cut-off is in line with clinical practice.

\section{Diagnostic criteria}

Variables included in the diagnostic guidelines are detailed in table 1 . The NINDS-AIREN and ADDTC criteria distinguish between probable and possible VaD. Criteria for probable dementia are presented in table 1. Patients were classified as having possible dementia, according to the NINDS-AIREN and ADDTC criteria, if brain imaging data to confirm definite CVD were missing, or in the absence of a clear temporal relationship between dementia and stroke. 


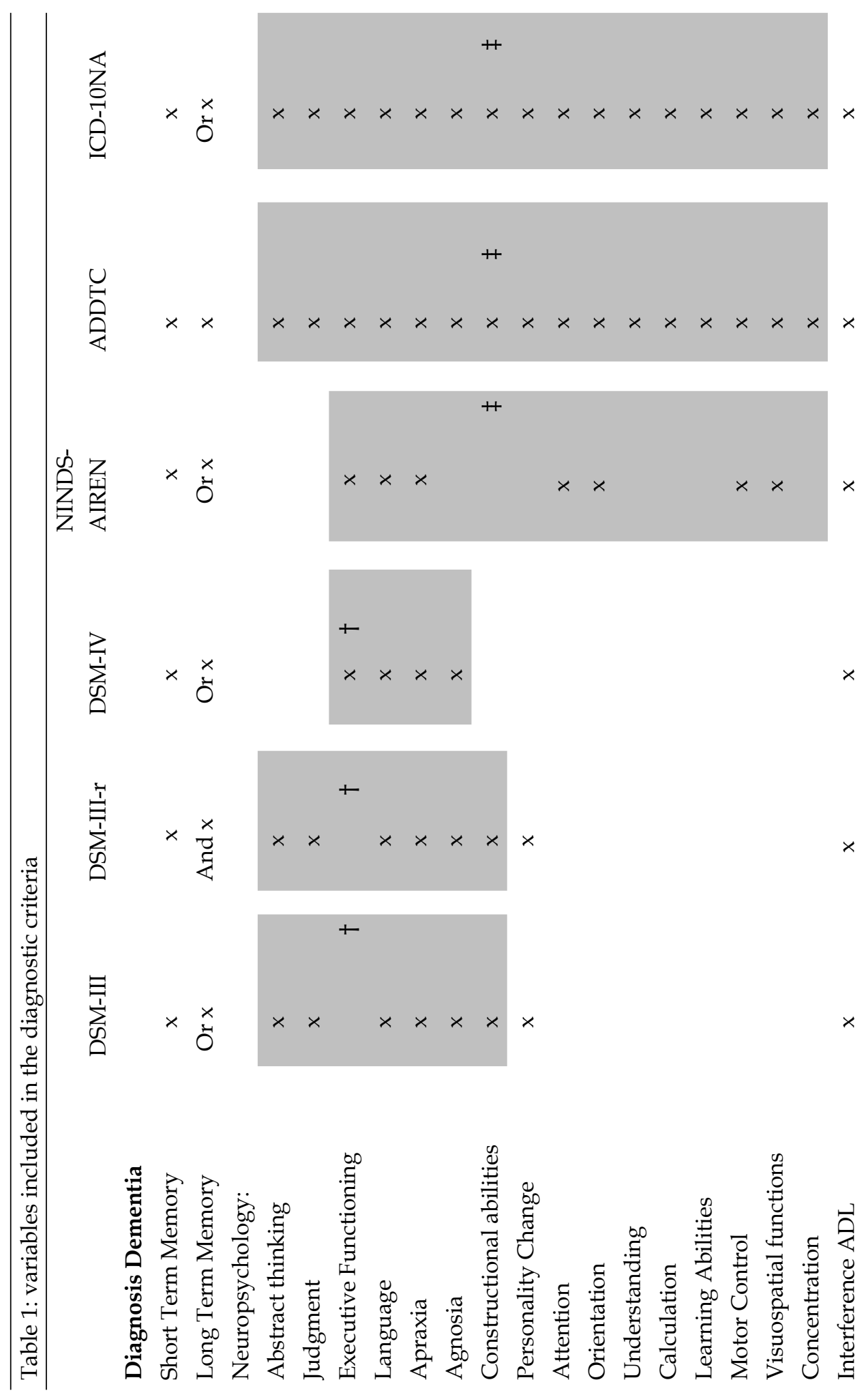




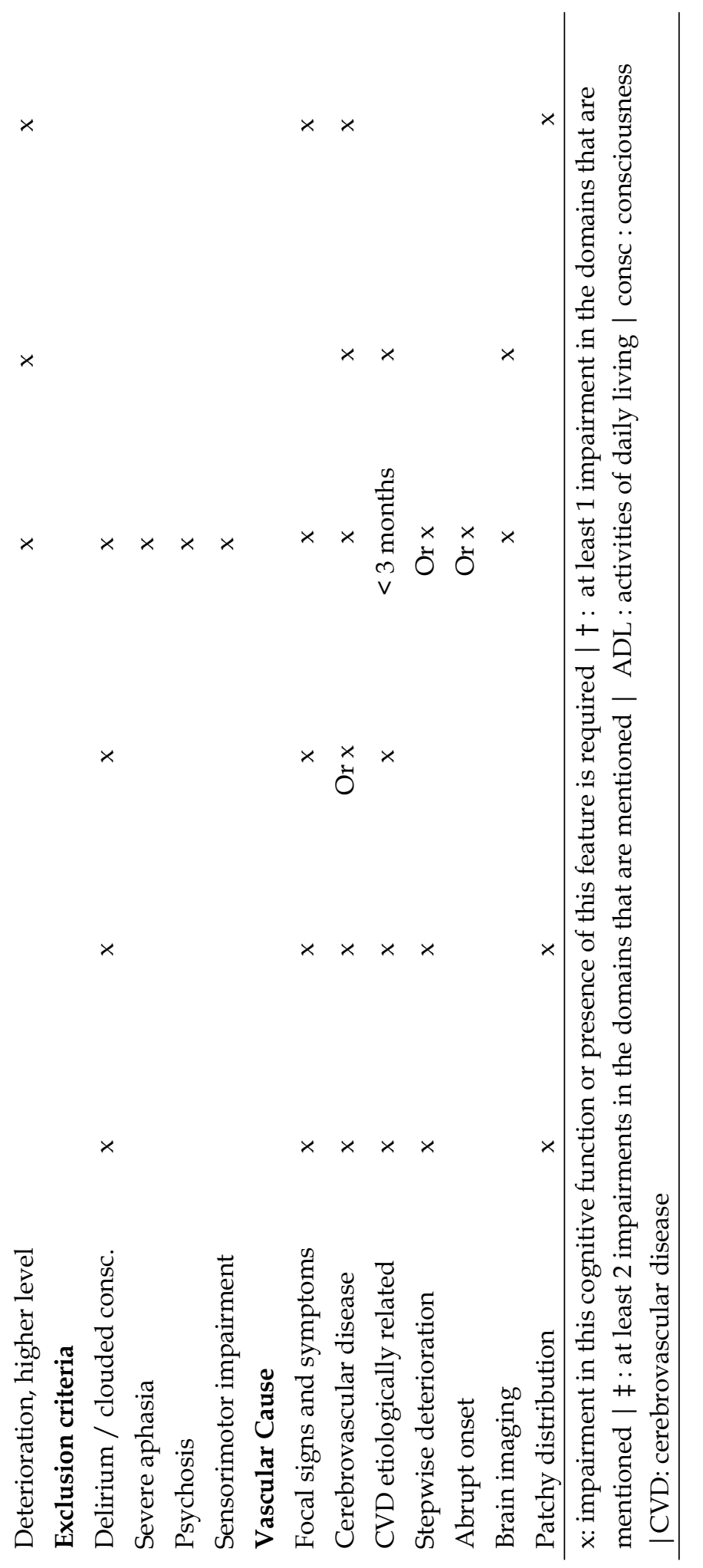




\section{Clinical assessment}

All patients underwent a structured medical and neurological assessment. This examination included medical history, clinical and neurological examination, laboratory examinations, and brain CT. A neurologist recorded the following features on CT: side of the symptomatic infarct (left or right hemisphere); stroke type (lacunar or territorial); presence of white matter lesions; presence of silent infarcts; and degree of brain atrophy. CT was performed on the day of admission or the next day. These data were entered in the Maastricht Stroke Register (MSR). A neuropsychologist assessed the patients, using a comprehensive test battery, consisting of the following tests: CAMCOG, observation scale of the CAMDEX, Concept Shifting Test (CST), Stroop Colour Word Test (SCWT), Auditory Verbal Learning Test (AVLT), Groninger Intelligence Scale (GIT), and the Interview for Deterioration in Daily living activities in Dementia (IDDD) [29, 38, 39, 42, 44, $45,47,101]$. This battery and the IDDD covered all relevant cognitive domains for the diagnosis of dementia. It was administered at each assessment period. Table 2 presents the cognitive domains assessed by the tests and the definitions of impairment. If memory could not be assessed with the AVLT, memory deficit was defined on the basis of the CAMCOG memory scale. Interference with daily activities was based on the IDDD: it was considered present if informants indicated that patients needed help in activities of daily living. If patients had physical disabilities, interference in activities of daily life was evaluated using the cognitive items of the IDDD, and not solely on the physical disabilities alone. Missing data were imputed as described in an earlier report [101]. 


\begin{tabular}{|c|c|c|}
\hline Cognitive Domain & Tests applied & Impairment \\
\hline Memory & $\begin{array}{l}\text { AVLT Or } \\
\text { CAMCOG: } \\
\text { memory remote and recent }\end{array}$ & $\begin{array}{l}<10 \text { th percentile norm group } \\
<80 \% \text { maximum score }(19)\end{array}$ \\
\hline Abstract thinking & $\begin{array}{l}\text { CAMCOG: } \\
\text { similarities }\end{array}$ & $<80 \%$ maximum score $(7)$ \\
\hline Judgment & $\begin{array}{l}\text { Observation scale } \\
\text { CAMDEX }\end{array}$ & $\geq 2$ observations present \\
\hline Executive functioning & $\begin{array}{l}\text { Interference score SCWT } \\
\text { and CST }\end{array}$ & $<10$ th percentile norm group \\
\hline Language & $\begin{array}{l}\text { CAMCOG: } \\
\text { understanding and expres- } \\
\text { sion }\end{array}$ & $<80 \%$ maximum score $(24)$ \\
\hline $\begin{array}{l}\text { Apraxia or motor } \\
\text { control }\end{array}$ & $\begin{array}{l}\text { CAMCOG: } \\
\text { copy a figure, motor abili- } \\
\text { ties }\end{array}$ & $<80 \%$ maximum score $(10)$ \\
\hline $\begin{array}{l}\text { Constructional or } \\
\text { Visuospatial }\end{array}$ & $\begin{array}{l}\text { GIT: } \\
\text { Mental rotation }\end{array}$ & $<10$ th percentile norm group \\
\hline $\begin{array}{l}\text { Attention or } \\
\text { Concentration }\end{array}$ & $\begin{array}{l}\text { CAMCOG: } \\
\text { counting backward }\end{array}$ & $<80 \%$ maximum score $(7)$ \\
\hline Orientation & $\begin{array}{l}\text { CAMCOG: } \\
\text { place, person and time }\end{array}$ & $<80 \%$ maximum score $(8)$ \\
\hline Understanding & $\begin{array}{l}\text { CAMCOG: } \\
\text { following instruction, } \\
\text { define }\end{array}$ & $<80 \%$ maximum score $(7)$ \\
\hline Calculation & GIT: doing sums & $<10$ th percentile norm group \\
\hline Learning Abilities & $\begin{array}{l}\text { CAMCOG: } \\
\text { remembering/recognition }\end{array}$ & $<80 \%$ maximum score $(14)$ \\
\hline Interference & IDDD & Need often help in daily live \\
\hline \multicolumn{3}{|c|}{$\begin{array}{l}\text { AVLT: Auditory verbal learning test | SCWT: Stroop Colour Word Test | } \\
\text { CST: Concept Shifting | GIT: Groninger intelligence scale | IDDD: Interview for } \\
\text { Deterioration in Daily Living Activities in Dementia }\end{array}$} \\
\hline
\end{tabular}

Information about pre-stroke and present cognitive functioning was obtained from a knowledgeable informant by means of a structured interview, based on the DSM-IV criteria for dementia. All patients had a informant (in 59.5\% patients a partner, in $28.2 \%$ a son or daughter, in $3.6 \%$ a close friend, in $3.1 \%$ a 
sibling, and in 5.6\% a professional caregiver provided the information). Fifteen point five percent of the informants reported memory problems present before stroke, however, these were not that severe that they cause interference in daily life. For the analysis we used the data from the neuropsychological evaluation. The DSM-III, DSM-III-R and ICD-10-NA all include in their diagnostic criteria a patchy distribution of deficits and personality changes, but these terms are not clearly operationalized, and therefore we did not include these aspects. Agnosia could not be assessed with our test battery. The ICD-10-NA, NINDS-AIREN, and ADDTC criteria require that dementia is defined by a cognitive decline from a previously higher level of functioning. Because none of our patients were demented before stroke, this definition was applicable to all patients with post-stroke dementia. Focal neurological signs or symptoms were present in all patients because they had all been referred to the neurological department for stroke. These signs were confirmed by medical examination and CT.

\section{Statistical Analysis}

The effect of diagnostic criteria on the prevalence and cumulative incidence of VaD over a 2-year period was investigated in 194 patients who had had a stroke. Prevalence was further investigated in different age-specific groups according to the median split, two age groups were formed: age lower than (or equal to) 70 , and age older than 70 years. Agreement between the criteria was calculated with the use of $\mathrm{K}$ statistics, which indicate how much better the agreement is than that due to chance alone. The following guidelines have been suggested for interpreting K scores: 0.00 to 0.20 indicates slight agreement; 0.21 to 0.40 fair agreement; $0.41-0.60$ moderate agreement; 0.61 to 0.81 substantial agreement; and 0.81 to 1.00 almost perfect agreement [102].

\section{Results}

One hundred and ninety-four patients were examined 1 month after stroke: 107 men $(55.2 \%)$ and 87 women $(44.8 \%)$, with an average age of $68.2(\mathrm{SD}=12.4)$ years and an average baseline (i.e. one month evaluation) MMSE-score of 25.5 $(\mathrm{SD}=3.5)$. At 6 months after stroke $170(87.6 \%)$ patients participated (4 died, 6 refused temporarily, 14 refused further participation, and 1 patient was untraceable), at 12 months 157 (80.9\%) patients participated (8 died, 6 refused temporary, 6 refused further participation, and 1 patient was untraceable), and at 24 months after stroke $144(73.7 \%)$ patients participated (15 died, 1 was too ill, 4 refused further participation, and 1 patient was untraceable). Prevalence rates and incidence rates are presented in table 3. 
Table 3: Number of patients diagnosed with vascular dementia according to different diagnostic systems; Prevalence and cumulative incidence rates

\begin{tabular}{llllll} 
DSM-III & DSM-III-r & DSM-IV & NINDS- & ADDTC & ICD-10-NA \\
$\mathrm{N}(\%)$ & $\mathrm{N}(\%)$ & $\mathrm{N}(\%)$ & AIREN & $\mathrm{N}(\%)$ & $\mathrm{N}(\%)$ \\
& & & $\mathrm{N}(\%)$ & & \\
\hline
\end{tabular}

\begin{tabular}{lrlllllllllll}
\hline $\begin{array}{l}\text { Prevalence } \\
\text { Baseline }\end{array}$ & 38 & $(19.6)$ & 30 & $(15.5)$ & 35 & $(18.0)$ & 22 & $(11.3)$ & 31 & $(16.0)$ & 39 & $(20.1)$ \\
\hline Cumulative Incidence & & & & & & & & & & \\
6 months & 9 & $(4.6)$ & 9 & $(4.6)$ & 8 & $(3.6)$ & 6 & $(3.1)$ & 5 & $(2.6)$ & 10 & $(5.2)$ \\
12 months & 14 & $(7.2)$ & 11 & $(5.7)$ & 12 & $(6.2)$ & 8 & $(4.1)$ & 8 & $(4.1)$ & 14 & $(7.2)$ \\
24 months & 17 & $(8.8)$ & 12 & $(6.2)$ & 20 & $(9.3)$ & 14 & $(7.2)$ & 10 & $(5.2)$ & 18 & $(9.3)$ \\
\hline
\end{tabular}

The prevalence of VaD was highest with the ICD-10-NA criteria, followed by the DSM-III criteria, and was lowest with the NINDS-AIREN criteria.

The prevalence of possible VaD was $8.7 \%$ as defined by the NINDS-AIREN and $26.2 \%$ as defined by the ADDTC criteria. The incidence of $\mathrm{VaD}$ ranged from 4 to $5 \%$ for all criteria. All diagnostic criteria reported the highest incidence at 6 months after stroke. The cumulative incidence increased over the 2-year period.

Age specific prevalence indicated that by increasing age, the prevalence of poststroke VAD also increased. Prevalence of dementia for people younger than 70 varies from $6.2 \%$ by the NINDS-AIREN criteria to $11.3 \%$ by the ADDTC criteria. Prevalence of dementia for people older than 70 varies from $14.1 \%$ by the NINDS-AIREN criteria to $29.3 \%$ by the ICD-10-NA criteria.

There was a strong agreement between the diagnostic systems (Table 4), with the exception of the ADDTC.

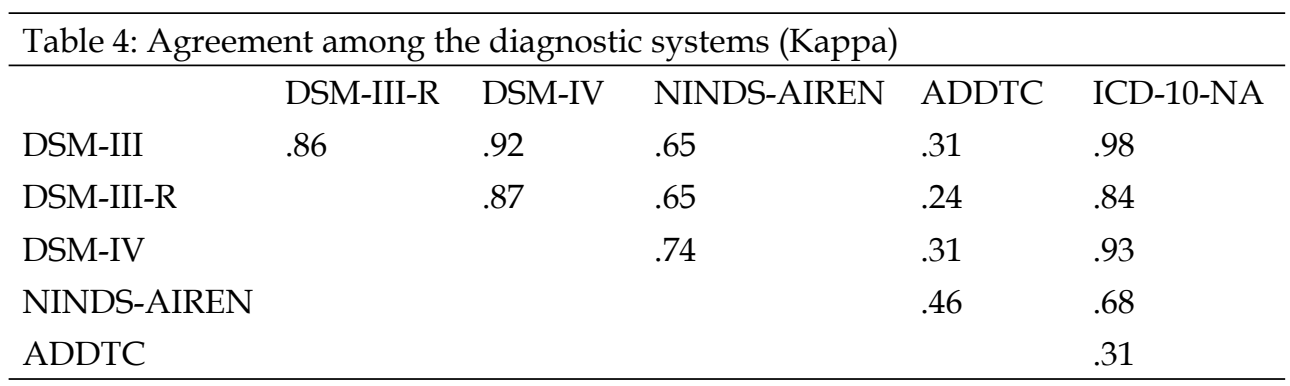




\section{Discussion}

The present study evaluated the impact of different diagnostic criteria on the prevalence and cumulative incidence of $\mathrm{VaD}$ in a series of 194 stroke patients. Results showed that the prevalence and cumulative incidence of $\mathrm{VaD}$ were largely dependent on the diagnostic criteria used. In line with other studies, the prevalence rates were lowest when the NINDS-AIREN criteria were used and highest when the DSM-III and ICD-10-NA criteria were used [2, 4, 5]. According to the findings of a recent neuropathological study, the NINDS-AIREN criteria are more stringent than those of the ADDTC [103], which would explain the lower prevalence of $\mathrm{VaD}$ according to the NINDS-AIREN criteria.

This study is the first to show that the cumulative incidence of dementia after stroke is affected by the diagnostic criteria used. The incidence of post-stroke $\mathrm{VaD}$ was lowest when the ADDTC criteria were used and highest when the DSM-III and ICD-10-NA criteria were used. This low incidence seems a bit contraindicatory as the ADDTC does not require a memory deficit. The ADDTC criteria could best be compared to the NINDS-AIREN criteria, in that they both are, compared to the other criteria, very restrictive in defining the etiology of the dementia. However, compared to the NINDS-AIREN criteria, the ADDTC criteria have a high sensitivity and thus most cases of post-stroke VaD were diagnosed at baseline, leaving fewer incident cases [103, 104]. Other studies have reported similar incidence rates $[32,98,99,105]$ and that the highest incidence of $\mathrm{VaD}$ occurs directly after stroke. Because it may be possible to prevent or delay dementia caused by vascular disorders [106] it is important to use sensitive criteria. For this reason, we recommend the use of the ADDTC criteria. In our study, patients who were diagnosed as being demented according to the ADDTC criteria would be classified as 'cognitive impairment no dementia', (or vascular cognitive impairment, no dementia) when applying the NIND-AIREN criteria.

In general, the agreement between diagnostic criteria was high, with the exception of the ADDTC criteria. This is probably because the ADDTC criteria do not require a memory deficit for the diagnosis 'dementia' and are thus less restrictive than the other diagnostic criteria. In an earlier study, we found that memory was impaired in only $20 \%$ of the stroke patients whereas other cognitive impairments were more common $[29,101]$. So the broader focus of the ADDTC criteria is to be preferred to the narrower focus of criteria for poststroke $\mathrm{VaD}$ that require a memory impairment. In contrast, the ADDTC criteria are more stringent than the DSM or ICD-10-NA criteria in terms of defining the etiology, because they require evidence of a cerebrovascular deficit visualised on a brain scan. Other studies reported lower agreement between criteria [2, $4,107]$, which may be because our study population was relatively homogene- 
ous. Patients with severe aphasia, severe cognitive deficits, and with recurrent strokes were excluded. This probably led to an underestimation of the number of patients that became demented. Although most of the criteria showed a high level of agreement, they still have shortcomings. For example, in all criteria, except those of the ADDTC, the diagnosis of dementia is based on criteria for $\mathrm{AD}$, which implies that a memory impairment has to be present. This emphasis on memory is not justified in dementia of the vascular type because other cognitive functions, such as executive functioning and mental speed, are often more disturbed than memory $[29,76,101]$. Secondly, these criteria do not allow for a distinction to made between dementia caused by cerebrovascular disease and Alzheimer type dementia with comorbid cerebrovascular disease [30]. Thirdly, the temporal relationship between dementia and cerebrovascular disease is difficult to establish in some cases of cerebrovascular disease. The temporal relation is clear-cut in the case of stroke whereas white matter disease or silent infarcts often go unnoticed. Cognitive disorders, related to these deficits may have an insidious onset with a progressive course and do not show a clear temporal relation with progression of cerebrovascular disease [27]. For these reasons, Bowler and Hachinski introduced a concept broader than VaD: Vascular Cognitive Impairment (VCI). In this concept 'vascular' refers to all causes of ischemic cerebrovascular disease and 'cognitive impairment' encompasses all levels of cognitive decline, from the earliest step to the most severe cognitive disorder. The authors did not specify criteria for this concept but they emphasized that new criteria should be evidence based and not consensus based [7]. The main purpose of the introduction of this concept was to identify patients with vascular pathology who are at risk for cognitive deterioration as early as possible.

Our study has some shortcomings. First, because of the study design, with testing at 1, 6, 12, and 24 months after stroke, we were not able to establish a precise temporal relationship between cerebrovascular disease and dementia. In case of the NINDS-AIREN criteria, this could have led to overestimation of the prevalence of $\mathrm{VaD}$ as these criteria specify that cognitive deficits should be present within 3 months after stroke, but in our study clinical information was only available at 1 , or at 6 months. Secondly, it is possible that some of our patients also had Alzheimer pathology and as such should have been diagnosed with mixed dementia instead of $\mathrm{VaD}$ [31]. Pohjasvaara reported that $28.4 \%$ of stroke patients had mixed dementia 3 months after stroke. Because we excluded patients with recurrent stroke and those with severe cognitive deficits directly after the stroke, we probably excluded the patients in whom dementia was developing before they had the stroke and, consequently, the percentage of mixed dementia would probably be lower in our study than in other studies. Thirdly, we used $\mathrm{CT}$ to detect brain damage instead of the more sensitive technique of magnetic resonance imaging (MRI). Our study was clinically based, and MRI data were available from routine practice in only a few cases. However, CT may be more 
specific than MRI and as such the chance of false positive findings is likely to be low. On the other hand the chance of false negatives may be a bit higher, which could have led to an underestimation of our results.

In conclusion, the prevalence and incidence of post-stroke $\mathrm{VaD}$ are affected by the diagnostic criteria used. However, irrespective of the criteria used, most cases of $\mathrm{VaD}$ are diagnosed directly after the stroke. 


\section{Predictive accuracy of MCI-subtypes for Alzheimer's disease and vascular dementia in subjects with mild cognitive impairment: a 2-year follow-up study}

\section{Adapted from:}

Rasquin SMC, Verhey FRJ, Lousberg, R, Visser PJ, Lodder J. Predictive accuracy of MCI-subtypes for Alzheimer's disease and vascular dementia in subjects with mild cognitive impairment: a 2-year follow-up study. Dementia and Geriatric Cognitive Disorders. In press. 


\section{Abstract}

Background: The aim of this study was to investigate the prognostic accuracy of different subtypes of mild cognitive impairment (MCI): amnestic MCI, multiple domains $\mathrm{MCI}$, and single non-memory domain $\mathrm{MCI}$, for the development of Alzheimer's dementia (AD) and vascular dementia (VaD).

Methods: Nondemented patients from a memory clinic cohort $\mathrm{N}=118$, and a stroke cohort $\mathrm{N}=80$ (older than 55 years and with a cognitive impairment).

Results: 'Multiple domains $\mathrm{MCI}^{\prime}$ had the highest sensitivity for both AD (80.8\%) and $\mathrm{VaD}(100 \%)$, and 'amnestic $\mathrm{MCI}^{\prime}$ had the highest specificity $(85.9 \%$ for AD, $100 \%$ for $\mathrm{VaD})$. The positive predictive value was low for all subtypes (0.0 $32.7 \%)$, whereas the negative predictive value was high $(72.8-100 \%)$.

Conclusions: The subtype 'multiple domains $\mathrm{MCI}^{\prime}$ has high sensitivity in identifying people at risk for developing $\mathrm{AD}$ or $\mathrm{VaD}$. The predictive accuracy of the $\mathrm{MCI}$ subtypes was similar for both $\mathrm{AD}$ and $\mathrm{VaD}$. 


\section{Introduction}

Mild Cognitive Impairment (MCI) is a term referring to persons who do not fulfil criteria for dementia, but who exhibit some form of cognitive impairment. The most common causes of MCI are age-related cognitive impairment, and the predementia stages of Alzheimer's disease (AD), vascular dementia (VaD). It has recently been proposed that specific subtypes of $\mathrm{MCI}$ may be useful to distinguish between these three conditions [81]. For example, subjects with isolated memory impairment (amnestic MCI) may be at increased risk of developing $\mathrm{AD}$, while subjects with impairments in multiple cognitive domains (multiple domains $\mathrm{MCI}$ ) may be at increased risk of developing $\mathrm{VaD}$, and patients with a single non-memory domain impairment may be at increased risk of develo-ping fronto-temporal lobe dementia [81]. This proposal has not yet been tested in a clinical setting, but a recent population based study failed to find support for the proposal [108].

The aim of the present study is to investigate whether 'amnestic $\mathrm{MCI}^{\prime}$, 'multiple domains $\mathrm{MCI}^{\prime}$, and 'single non-memory domain $\mathrm{MCI}^{\prime}$ are diagnostic entities for the predementia stage of $\mathrm{AD}$ and $\mathrm{VaD}$ in two separate cohorts (memory clinic and stroke cohort) of subjects with MCI. As there is controversy about the emphasis of an (isolated) memory impairment in the concept MCI, [80, 109, 110], we further subdivided the subtype 'multiple domains $\mathrm{MCI}^{\prime}$ into: 'multiple domains MCI with memory impairment included' (memory+) and 'multiple domain MCI with memory impairment excluded' (memory-).

\section{Methods}

\section{Patients}

Two clinical cohorts of nondemented, cognitively impaired subjects were available for this study: a cohort of patients with memory complaints who contacted the Memory Clinic of the University Hospital Maastricht, and a cohort of firstever stroke patients from the stroke unit of the same hospital. Patients from the memory clinic cohort were participants of a longitudinal study of Mild Cognitive Impairment which started in 1990 [111]. The stroke patients were participants of a longitudinal study called CODAS (COgnitive Disorders After Stroke) $[29,101]$.

Inclusion criteria for this study were; age older than 55 years, a cognitive impairment affecting at least 1 out of 9 cognitive domains (see below), no interference with daily activities (based on a questionnaire for the informant), and follow- 
up longer than 2 years. Exclusion criteria were dementia at baseline (defined according to DSM-IV), an MMSE score < 15 (to ensure valid neuropsychological testing), and the presence of any somatic or psychiatric disorder that could be related to the cognitive impairment, except a first-ever stroke in the CODAS cohort. Patients from the memory clinic cohort were excluded if they had cerebrovascular disorders, defined by a score on the Hachinski Ischemic Scale of 5 or higher [20]. In the CODAS cohort, patients with a previous stroke or a stroke in the cerebellum or brainstem were excluded.

Patients from the MCI cohort who were excluded because the follow-up period was too short $(\mathrm{N}=44)$ were older than the included patients, but MMSE scores, sex ratio and level of education were not different. Patients from the stroke cohort who were excluded because the follow-up period was too short $(\mathrm{N}=34)$ were older and had lower baseline MMSE scores than the included patients, but sex ratios and level of education were not different.

\section{Procedure}

Patients from the longitudinal MCI study were tested at baseline and 24 months later. Patients from the CODAS study were tested at 1, 6, 12, and 24 months after stroke. This report used the data from the baseline and 24-months assessments.

\section{Cognitive Domains}

Table 1 presents the cognitive domains investigated.

The neuropsychological tests used have been described extensively in earlier reports [29, 49, 101].

The choice of the domains studied, was based on the criteria for VaD proposed by the National Institute of Neurological Disorders and Strokes - Association Internationale pour la Recherche et l'Enseignement en Neurosciences (NINDSAIREN) [24]. 'Memory' was evaluated with the Auditory Verbal Learning Test (AVLT) [42]; 'executive functioning' with a combination of the interference score of the Stroop Colour Word Test (SCWT) [45] and with the interference score of either the Concept Shifting Test (CST) [82] or the Trail Making Test (TMT) [43]; 'calculation' and 'visuospatial abilities' with subscales of the Groninger Intelligence Scale (GIT) [47]; and 'orientation', 'attention', 'praxis', and 'language' with subtests of the MMSE. [41]. In addition to the 8 cognitive domains, we included also tests to assess 'mental speed', as this aspect may be of relevance according to previous studies [29,56]. 'Mental speed' was evaluated with a combination of the scores of the first part of the SCWT [45] and with the scores on the first and second part of either the CST [82] or the TMT [43]. 
Both cohorts were administered the same neuropsychological battery with one exception: patients from the memory clinic cohort completed the TMT, whereas patients from the CODAS study completed the CST. Because the TMT and CST measure the same cognitive function and are highly correlated it was valid to assess the tests in each single cohort [82]. The performance of both cohorts was compared with that of a normative group from the MAastricht Aging Study (MAAS) [49]. The aim of the MAAS study is to investigate factors that predispose to normal cognitive aging in a cohort of healthy elderly volunteers from the southern part of the Netherlands. Normative data from this study were stratified according to age, sex, and educational level. Individual test scores were converted to standard Z-scores, in order to define impairment in the cognitive functions, using the means and standard deviations of the normative data (testscore patient - mean norm-group)/SD norm-group.

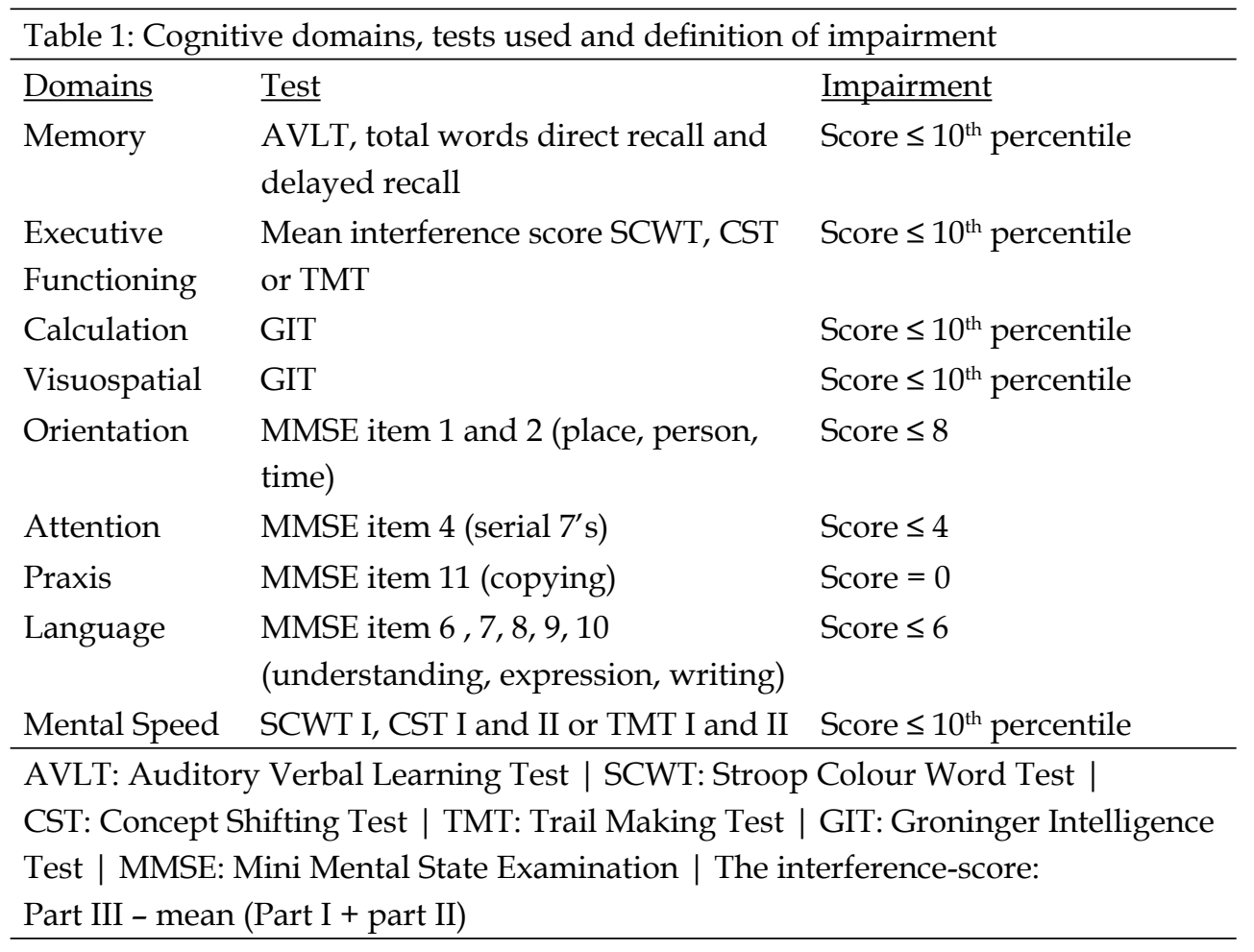

\section{Diagnosis of Dementia}

At 24 months, and without knowledge of the baseline data, two experienced clinicians independently evaluated patients regarding a diagnosis of dementia according to the DSM-IV criteria [23], based on available data, such as infor- 
mation about medical history, test performance, structured interview with an informant, and a clinical observation. Interference in daily activities due to cognitive impairments was evaluated by means of questionnaires about daily activities that were completed by the informant [38]. The diagnosis of AD was based on the NINCDS-ADRDA criteria and the diagnosis of VaD was based on the NINDS-AIREN criteria.

If there was a discrepancy in diagnosis a consensus meeting was arranged. If no consensus could be reached the patient was considered not demented. Agreement between the two clinicians was high $(\kappa=0.88 ; \mathrm{p}=.01)$.

\section{Diagnosis of MCI}

MCI was defined if patients had: 1) an impairment in at least one of the nine cognitive domains; 2) no of interference in daily activities; 3 ) no dementia according to the DSM-IV. A cognitive impairment was defined as a score lower than the 10the percentile of the z-scores, based on the norm-scores, compared to the norm-group (equivalent of $1.28 \mathrm{SD}$ below the norm-scores) or a MMSE a score lower than $80 \%$ of the maximum score on the relevant scale.

The descriptions proposed by Petersen et al. [81] were used to subclassify MCI. Patients were classified as 'amnestic MCI' if they had a memory impairment, but were performing otherwise well (z-score $>1.28$ compared to the normgroup) on other indexes of cognitive function. Patients were classified as 'multiple domains $\mathrm{MCI}^{\prime}$ if they had impairments on two or more domains. Patients were classified as 'multiple domains MCI memory+' if they had impairments on two or more domains, one of which was memory impairment. In fact this concept largely corresponds with the concept of 'cognitive impairment no dementia' (CIND). Moreover we made a subclassification in 'multiple domains MCI memory-' if patients had impairments on two or more domains but no memory impairment

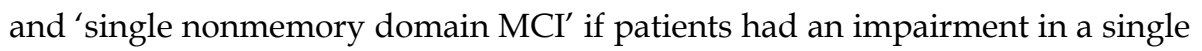
domain, other than memory.

\section{Statistical Analyses}

Descriptive statistics were used for the demographic variables and the number of patients that became demented. Baseline differences, between the two groups, with regard to the number of cognitive impairments were tested with a Chi Square Test or T-test. The validity and predictive value of $\mathrm{MCI}$ subtype as being diagnostic for the development of dementia were determined with sensitivity, specificity, and predictive values. Analyses were performed with the Statistical Package for Social Sciences version 10 (SPSS-10) for Macintosh. 


\section{Results}

In total 118 patients from the memory clinic cohort and 80 patients from the stroke cohort were included in this study. Patients from the stroke cohort had a lower educational level, were older, and had lower baseline MMSE score than patients from the memory clinic cohort (Table 2).

Table 2: Demographic characteristics of the two cohorts

\begin{tabular}{llll} 
& \multicolumn{1}{l}{ Memory-clinic Cohort } & Stroke cohort \\
$\mathrm{N}$ & 118 & 80 \\
Age (mean, SD) & $64.8(7.5)$ & $70.4(7.8)^{*}$ \\
Level of education \% Low & 23.7 & $56.3^{*}$ \\
Sex \% Female & 48.3 & $55.0 \%$ \\
MMSE-score (mean, SD) & $27.3(2.2)$ & $26.3(2.4)^{*}$ \\
\hline
\end{tabular}

${ }^{*} \mathrm{p}<.05$ 
At baseline compared with patients from the memory clinic cohort, patients from the stroke cohort more often had an impairment in calculation $\left(\chi^{2}=16.3\right.$, $\mathrm{df}=1, \mathrm{p}=.01)$, visuospatial functioning $\left(\chi^{2}=14.3, \mathrm{df}=1, \mathrm{p}=.01\right)$, attention $\left(\chi^{2}\right.$ $=8.8, \mathrm{df}=1, \mathrm{p}=.01)$, language $\left(\chi^{2}=8.7, \mathrm{df}=1, \mathrm{p}=.01\right)$, and praxis $\left(\chi^{2}=15.2, \mathrm{df}\right.$ $=1, \mathrm{p}=.01)$, but less often an impairment in memory $\left(\chi^{2}=34.4, \mathrm{df}=1, \mathrm{p}=.01\right)$ (Figure 1).

Figure 1: Percentage of patients with an impairment

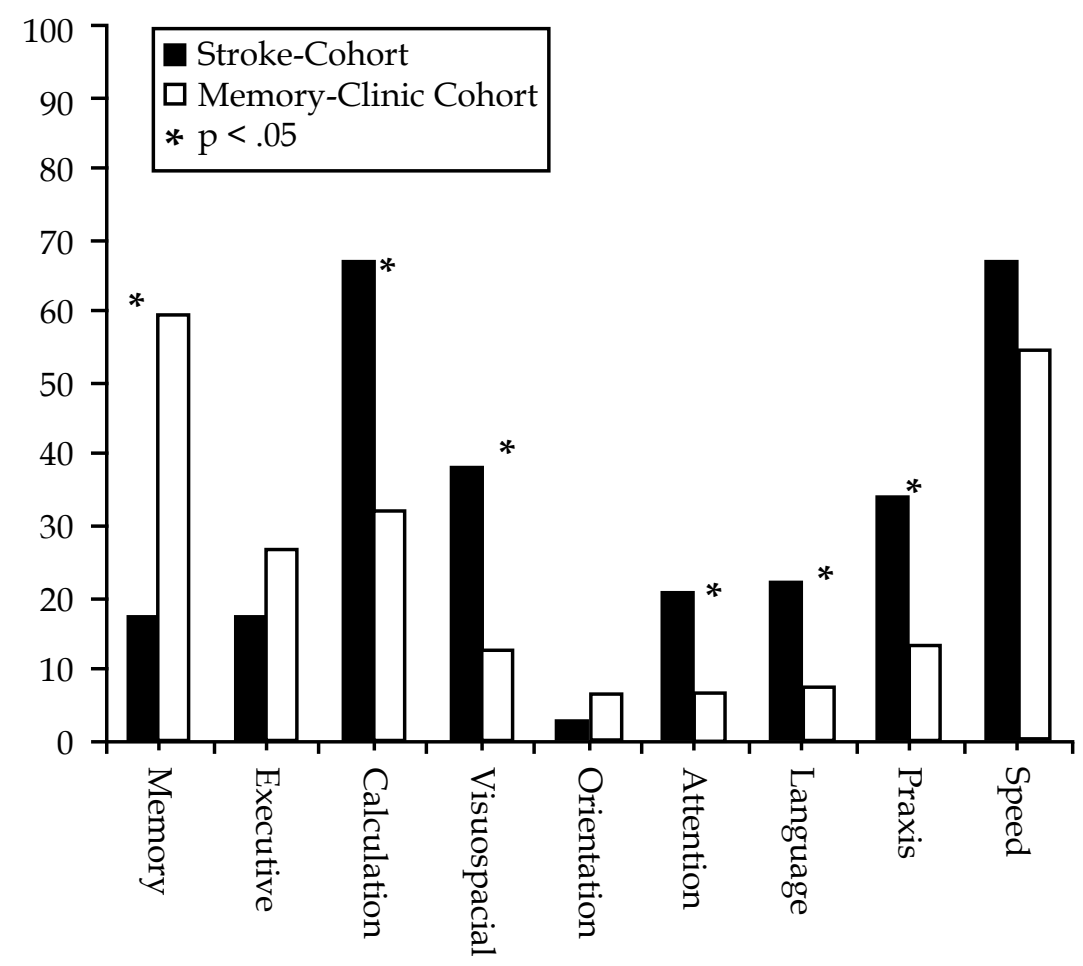


In both cohorts most patients $(63.5 \%$ and $73.8 \%$ respectively) had 'multiple domains MCI' (Table 3).

In the memory clinic cohort, 26 patients were classified as demented $(22.0 \%)$ at follow-up, all being diagnosed with AD according to the NINCDS-ADRDA. In the stroke cohort only 5 patients had dementia at follow-up (6.3\%), all of whom were diagnosed with $\mathrm{VaD}$ according to the NINDS-AIREN criteria.

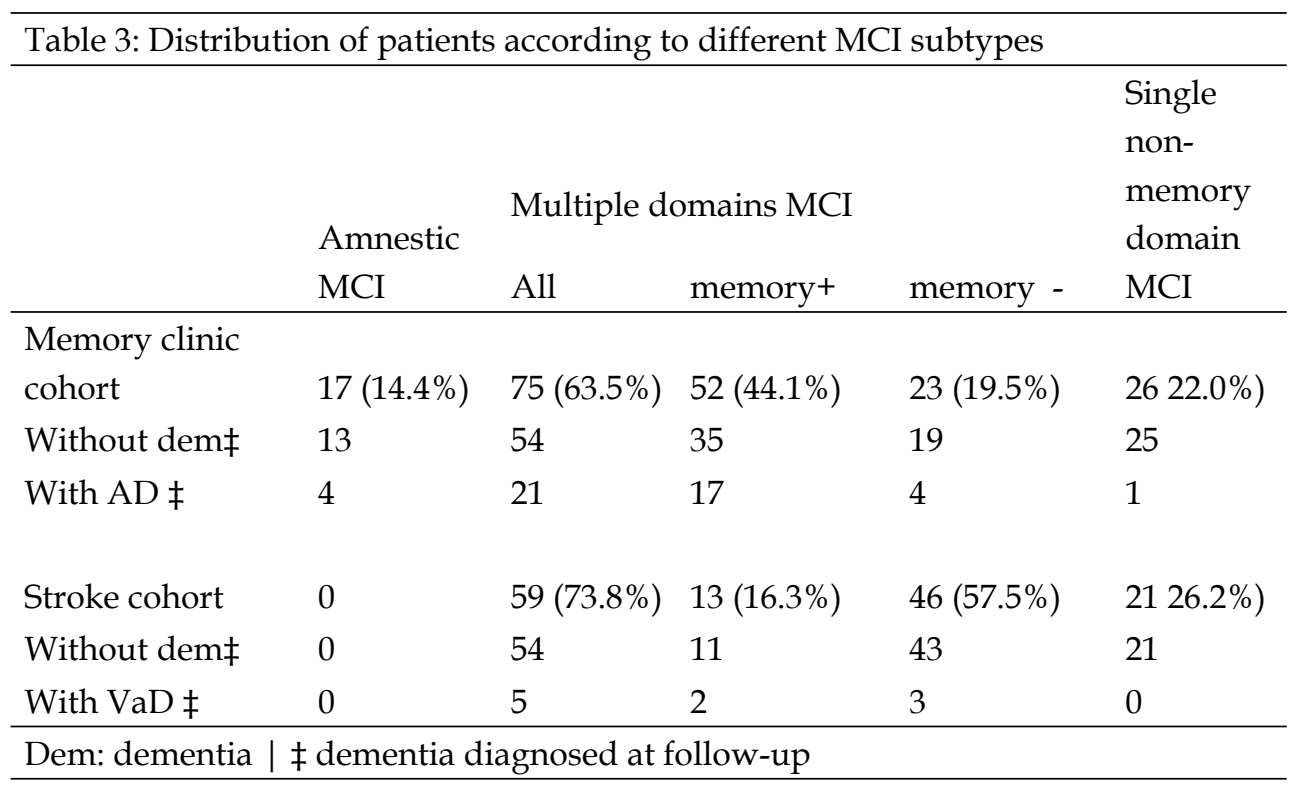

The sensitivity and specificity of the four MCI subtypes for the diagnosis of either $\mathrm{AD}$ or $\mathrm{VaD} 2$ years later are presented in table 4. The subtype 'multiple domains $\mathrm{MCI}^{\prime}$ had high sensitivity for the development of both $\mathrm{AD}$ and $\mathrm{VaD}$. Specificity rates were highest for the amnestic type in both cohorts. For all MCI subtypes the positive predictive value was low (range 0.0 to $32.7 \%$ ) but the negative predictive value was higher for all MCI subtypes (range 72.8 - 100\%). 
Table 4: Sensitivity and specificity for the four MCI-sybtypes for the development of either $\mathrm{AD}$ or $\mathrm{VaD}$

\begin{tabular}{|c|c|c|c|c|}
\hline & $\begin{array}{l}\text { Sensitivity } \\
(95 \% \mathrm{CI})\end{array}$ & $\begin{array}{l}\text { Specifictiy } \\
(95 \% \mathrm{CI})\end{array}$ & $\begin{array}{l}\text { PPV } \\
(95 \% \text { CI) }\end{array}$ & $\begin{array}{l}\text { NPV } \\
(95 \% \text { CI })\end{array}$ \\
\hline \multicolumn{5}{|l|}{ Memory clinic cohort } \\
\hline Amnestic MCI & $\begin{array}{l}15.4 \% \\
(0.02-0.29)\end{array}$ & $\begin{array}{l}85.9 \% \\
(0.79-0.93)\end{array}$ & $\begin{array}{l}23.5 \% \\
(0.03-0.44)\end{array}$ & $\begin{array}{l}78.2 \% \\
(0.7-0.86)\end{array}$ \\
\hline Multiple Domains All & $\begin{array}{l}80.8 \% \\
(0.66-0.96)\end{array}$ & $\begin{array}{l}41.3 \% \\
(0.31-0.51)\end{array}$ & $\begin{array}{l}28.0 \% \\
(0.18-0.38)\end{array}$ & $\begin{array}{l}88.4 \% \\
(0.79-.098)\end{array}$ \\
\hline $\begin{array}{l}\text { Multiple Domains MCI } \\
\text { memory+ }\end{array}$ & $\begin{array}{l}65.4 \% \\
(0.47-0.84)\end{array}$ & $\begin{array}{l}61.9 \% \\
(0.52-0.72)\end{array}$ & $\begin{array}{l}32.7 \% \\
(0.20-0.45)\end{array}$ & $\begin{array}{l}86.4 \% \\
(0.78-0.95)\end{array}$ \\
\hline $\begin{array}{l}\text { Multiple Domains MCI } \\
\text { memory- }\end{array}$ & $\begin{array}{l}15.4 \% \\
(0.02-0.29)\end{array}$ & $\begin{array}{l}79.3 \% \\
(0.71-0.88)\end{array}$ & $\begin{array}{l}17.3 \% \\
(0.02-0.33)\end{array}$ & $\begin{array}{l}76.8 \% \\
(0.68-0.85)\end{array}$ \\
\hline Single Nonmemory MCI & $\begin{array}{l}3.8 \% \\
(0.0-0.11)\end{array}$ & $\begin{array}{l}72.8 \% \\
(0.64-0.82)\end{array}$ & $\begin{array}{l}3.8 \% \\
(0.0-0.11)\end{array}$ & $\begin{array}{l}72.8 \% \\
(0.64-0.82)\end{array}$ \\
\hline \multicolumn{5}{|l|}{ Stroke Cohort } \\
\hline Amnestic MCI & $\begin{array}{l}0.0 \% \\
(0.0-0.0)\end{array}$ & $\begin{array}{l}100 \% \\
(1.0-1.0)\end{array}$ & $\begin{array}{l}0.0 \% \\
(0.0-0.0)\end{array}$ & $\begin{array}{l}93.8 \% \\
(0.88-0.99)\end{array}$ \\
\hline Multiple Domains All & $\begin{array}{l}100 \% \\
(1.0-1.0)\end{array}$ & $\begin{array}{l}28.0 \% \\
(0.18-0.38)\end{array}$ & $\begin{array}{l}8.5 \% \\
(0.01-0.16)\end{array}$ & $\begin{array}{l}100 \% \\
(1.0-1.0)\end{array}$ \\
\hline $\begin{array}{l}\text { Multiple Domains MCI } \\
\text { memory+ }\end{array}$ & $\begin{array}{l}40.0 \% \\
(0.0-0.83)\end{array}$ & $\begin{array}{l}85.3 \% \\
(0.77-0.93)\end{array}$ & $\begin{array}{l}15.4 \% \\
(0.0-0.35)\end{array}$ & $\begin{array}{l}95.5 \% \\
(0.91-1.0)\end{array}$ \\
\hline $\begin{array}{l}\text { Multiple Domains MCI } \\
\text { memory- }\end{array}$ & $\begin{array}{l}60.0 \% \\
(0.17-1.03)\end{array}$ & $\begin{array}{l}42.7 \% \\
(0.31-0.54)\end{array}$ & $\begin{array}{l}6.5 \% \\
(0.0-0.14)\end{array}$ & $\begin{array}{l}94.1 \% \\
(0.86-1.0)\end{array}$ \\
\hline Single Nonmemory MCI & $\begin{array}{l}0.0 \% \\
(0.0-0.0)\end{array}$ & $\begin{array}{l}73.8 \% \\
(0.62-0.82)\end{array}$ & $\begin{array}{l}0.0 \% \\
(0.0-0.0)\end{array}$ & $\begin{array}{l}92.1 \% \\
(0.84-0.99)\end{array}$ \\
\hline
\end{tabular}

To investigate which individual cognitive domain had the highest predicitive value we analysed post hoc predictive value for the development of either $\mathrm{AD}$ or $\mathrm{VaD}$ for each separate domain. Orientation had the highest positive predictive value for the development of $\mathrm{AD}(\mathrm{PPV}=62.5 \% \mathrm{CI}=.29-.96)$ and memory had the highest negative predictive value for the development of $\mathrm{AD}(\mathrm{NPV}=$ $91.5 \% \mathrm{CI}=.84-.99)$. For the development of $\mathrm{VaD}$, attention had the highest positive predictive value ( $\mathrm{PPV}=18.0 \% \mathrm{CI}=.00-.36)$ and both mental speed and executive functioning had the highest negative predictive value $\left(\mathrm{NPV}_{\text {speed }}=100 \%\right.$, $\mathrm{CI}=1 \cdot 0-1.0$ and $\left.\mathrm{NPV}_{\text {executive }}=100 \% \mathrm{CI}=1.0-1.0\right)$. 


\section{Discussion}

This is the first study to establish the prognostic value of the different MCI subtypes in a clinical population [81]. In the memory clinic cohort, the 'multiple domain $\mathrm{MCI}^{\prime}$ subtype was the most prevalent, and the 'amnestic $\mathrm{MCI}^{\prime}$ subtype the least. This suggests that isolated memory impairment is rare and that the amnestic MCI subtype has limited applicability in clinical practice, unlike 'multiple domains $\mathrm{MCI}^{\prime}$, which would appear to be a more relevant subtype [110]. Indeed, 'amnestic MCI' had a low sensitivity as a prognostic test for the development of $\mathrm{AD}$, whereas 'multiple domains $\mathrm{MCI}^{\prime}$ had a high sensitivity. Other investigators also found MCI or related concepts, such as CIND, to be valid with regard to the development of dementia (mostly AD) [112]. The low positive predictive value of the MCI subtypes found in our study may be because of the small number of cases of dementia diagnosed and the short follow-up. The results suggest that this subtype classification of MCI is of limited clinical relevance. The negative predictive value of these subtypes was high,

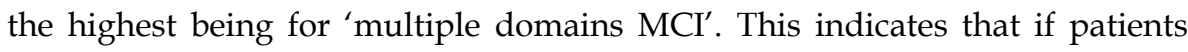

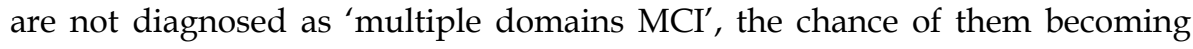
demented is low.

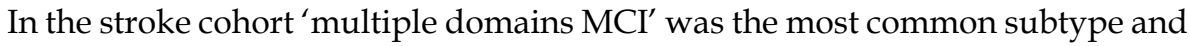
'amnestic $\mathrm{MCI}^{\prime}$ the least. 'Multiple domains $\mathrm{MCI}^{\prime}$ had the highest sensitivity and 'amnestic MCI' had the highest specificity for the predementia stage of $\mathrm{VaD}$. In their study of stroke patients, Ballard et al. did not find MCI and other related concepts to be useful as indicators of the transition phase to dementia after stroke [8]. Despite these negative results, nondemented patients with cerebro vascular diseases (CVD) and with mild cognitive deficits are at increased risk of developing dementia [10,12, 15, 78, 113].

In both cohorts, the 'multiple domains $\mathrm{MCI}^{\prime}$ subtype was the most prevalent, and in the stroke cohort, 'multiple domains MCI memory-' was more than 3 times more prevalent than the 'multiple domains MCI memory+'. These findings suggest that the cognitive profile of patients with cerebrovascular disease differs from that of patients with other disorders, which could also be concluded from the analysis with the individual cognitive domains in which patients fromt the MCI cohort more often had memory deficits which had also the highest negative predictive value, meaning that patients without a memory deficit had a lower chance of becoming demented than patients with a memory deficit. In the stroke cohort patients without executive functioning or without mental speed deficits had a lower chance of becoming demented compared to patients with these deficits. Busse et al. failed to find relation between MCI-subtypes and the development of dementia [108], which may be because they used a population-based design in which subjects with neurological disorders were excluded. 
As a result, subjects who were at higher risk of developing VaD were underrepresented [108].

This study has some shortcomings. First, patients from the two cohorts visited the hospital for different reasons: patients from the memory-cohort had memory complaints whereas patients from the stroke cohort had had a stroke. However, despite these differences in referral, the groups were similar with regard to cognitive functioning (MMSE score at baseline differed only 1 point), and inclusion and exclusion criteria. Secondly, the patients in the stroke cohort were tested four times, and those in the memory clinic cohort only two times, which may have led to differences caused by a learning effect. However, as dementia was primarily diagnosed on the basis of the clinical assessment, we consider this effect to be of limited relevance in our study. Thirdly, we diagnosed MCI and its subtypes based on psychometric definitions and not in combination with a clinical judgement. This is in contrast with the clinical diagnosis of $\mathrm{MCI}$, as described by Petersen [81]. Moreover, we did not take memory complaints into consi-deration, which means we may have overestimated the number of patients with $\mathrm{MCI}$ because other studies have shown that the prevalence of $\mathrm{MCI}$ increases when subjective cognitive complaints are not taken into consideration[108, 114]. Fourthly, because few patients were diagnosed with dementia, the power of the study was low, which may be a consequence of the exclusion of people with an MMSE score < 15. Lastly, the follow-up period was relatively short, which could have led to an underestimation of the positive predictive value of the various subtypes of MCI.

In conclusion, the subtype 'multiple domains $\mathrm{MCI}^{\prime}$ has the highest sensitivity in predicting both $\mathrm{AD}$ and $\mathrm{VaD}$. A high sensitivity in diagnosing patients who are at risk of developing dementia is desirable in the light of intervention possibilities.

We propose that patients who meet the diagnostic criteria of subtype 'multiple domains $\mathrm{MCI}^{\prime}$ are in the predementia stage and have a high risk of going on to develop dementia. However, the low positive predictive value indicates that other markers for the predementia stage are needed. 


\section{VIII}

\section{Predictors of reversible Mild Cognitive Impairment after stroke: a 2-year follow-up study}

Adapted from:

Rasquin SMC, Lodder J, Verhey FRJ. Predictors of reversible Mild Cognitive Impairment after stroke: a 2-year follow-up study. Journal of the Neurological Sciences. In press. 


\section{Abstract}

Background: Many studies have investigated Mild Cognitive Impairment (MCI) in the context of prodromal dementia, but few have investigated recovery from MCI. The aim of this study was to determine the prevalence of reversible MCI after stroke and to identify factors related to recovery.

Methods: One-hundred eighteen patients with a first-ever cerebral stroke were followed up for 2 years. Neuropsychological assessment was performed at 1 , 6,12 , and 24 months post-stroke. Possible predictors of reversible MCI were demographic variables, baseline MMSE-scores, presence of stroke risk factors, and CT-variables. Post-stroke MCI was diagnosed when there was a deficit in at least one cognitive domain, without their being demented. Recovery was considered when MCI was no longer present.

Results: wenty-four patients were classified as having reversible MCI and were compared with patients without recovery. Most patients recovered from MCI between the first and second assessments $(19.7 \%$ versus $13.1 \%$ and $2.0 \%$ later on). Higher baseline-MMSE scores and female sex were independent predictors of recovery $\left(\mathrm{OR}_{\text {High MMSE }}=9.9 ; \mathrm{OR}_{\text {female sex }}=2.8\right)$. Neither stroke-related risk factors nor CT-variables were predictors of favorable outcome.

Conclusion: About $20 \%$ of patients with post-stroke MCI recover from MCI. Higher MMSE-scores at baseline and female sex are independent predictors of this recovery. 


\section{Introduction}

Mild Cognitive Impairment (MCI) is a term generally used to describe the condition of patients who do not fulfil criteria for dementia, but who do exhibit some form of cognitive impairment. Patients with MCI are at risk of developing dementia, at a rate of $10-15 \%$ per year compared with, $1-2 \%$ per year in healthy controls [115]. Most studies of MCI have focused on the development of Alzheimer's Dementia (AD), whereas much less attention has been paid to MCI as the prodromal stage of Vascular Dementia (VaD). On average, per year about $10 \%$, of patients with MCI due to cerebrovascular disease develop VaD [15, 91, $113,116]$. Although most studies investigated $\mathrm{MCI}$ in the context of prodromal dementia, some have investigated recovery from $\mathrm{MCI}$, showing recovery from $\mathrm{MCI}$ or other related diagnostic entities to be in the range of $8-44 \%$ after 3 years [117-122]. Improvement in patients with MCI due to a cerebrovascular cause has also been reported, occurring in $2.7 \%$ to $31.0 \%$ of these patients [15, 113]. It is important to identify patients with MCI who will have a favorable outcome, because it may be inappropriate to start therapy in people with a high likelihood of spontaneous improvement. Indeed, spontaneous improvement of cognitive functioning after stroke has been reported [89, 91, 113, 123-125].

The aim of this study was to investigate the prevalence of reversible MCI after stroke and to identify factors that are related to recovery from the cognitive deficits. We hypothesized that patients who recovered from MCI would have less severe brain damage and fewer stroke-risk factors than those who did not recover.

\section{Methods}

\section{Patients}

This study is part of a larger project, called CODAS (COgnitive Disorders After Stroke). This is a longitudinal, prospective clinical study of cognitive functioning, risk factors for cognitive impairment, and the course of cognitive functioning after stroke [29]. Patients with a first-ever cerebral stroke (not located in the brainstem or cerebellum) were assessed clinically and neuropsychologically at $1,6,12$, and 24 months after stroke.

Patients who were admitted to the University Hospital Maastricht or who visited the outpatient clinic for stroke-related events between January 2000 and August 2001 were asked to participate in this study. An experienced neurologist diagnosed stroke based on clinical findings and Computed Tomography (CT)- 
scan findings.

Inclusion criteria were first-ever cerebral stroke, age older than 40 years, fluency in Dutch, no other neurological or psychiatric disorders, and a Mini Mental State Examination (MMSE) score $\geq 15$. Patients with severe cortical deficits were excluded, as were patients with no cognitive deficits at baseline and patients who were demented at baseline. Moreover, at least two follow-up assessments after stroke were required for each patient. Hundred-and-eighteen patients with MCI were included. The Medical Ethics Board of the University Hospital Maastricht approved the study, and all participants gave informed consent.

\section{Assessment of neuropsychological functioning}

Cognitive functioning was assessed using a neuropsychological test battery described elsewhere [29]. Memory, language, orientation, mental speed, attention, praxis, executive functioning, calculation and visuospatial functioning were assessed. These cognitive domains are mentioned in the NINDS-AIREN criteria [24]. Mental speed was also assessed. Cognitive functioning was compared with that of a norm group from the Maastricht Aging Study (MAAS), which investigates features that predispose to normal cognitive aging in older healthy volunteers from the region south Limburg. Norm tables are stratified according to age, sex, and level of education [49]. A score lower than the 10th percentile (a cut-off commonly used in clinical practice) of the norm group defined a cognitive deficit. MMSE was assessed in all patients [41]. Psychological symptoms were assessed with the Symptom Check List (SCL-90) [37]. This questionnaire is a multi-dimensional self-rating scale and covers a broad spectrum of psychological complaints, such as: anxiety, agoraphobia, depression, somatic complaints, interpersonal sensitivity, insufficiency of thinking, sleeping problems, and hostility. Patients were asked to indicate, on a 5-point scale, how much hinder they had experienced from certain psychiatric symptoms in the last week.

\section{Post-Stroke MCI}

Post-stroke MCI was diagnosed if patients fulfilled the following criteria: cognitive deficit on at least one of the cognitive functions; no interference of the cognitive deficits with daily activities, assessed by the IDDD [38]; and no dementia. Our definition of MCI is somewhat broader than the traditional criteria of Petersen et al., which originally focus on amnestic disorders [81]. Reversible post-stroke $\mathrm{MCI}$ was defined as a cognitive performance no longer below the norm set for age, sex, and educational level, at a later assessment period, i.e., the 
patients were no longer classified as having MCI.

Patients who recovered from $\mathrm{MCI}$ and who were without relapse, later on, were classified as having permanently reversible MCI.

\section{CT scan variables and medical history}

CT scans were evaluated by means of standardized criteria [53]. The following CT features were recorded: ischemic or hemorrhagic stroke, side of the lesion, stroke type (territorial or lacunar), presence of white matter lesions (WML), silent infarcts, and atrophy. The presence of WML around the frontal or occipital ventricular horns or in the centrum semi ovale was rated separately. No effort was made to quantify the degree of WML density at these sites. Silent brain infarction was defined as a low-density area on the CT scan, compatible with infarction but without a history of stroke as taken from the patient's history, from relatives, or any other accessible information. Also, the stroke symptoms at study entry had to be anatomically incompatible with such silent infarcts. The degree of atrophy was scored in a semi-quantitative way (none, mild, moderate, and severe), according to Leys et al. [55]. In short, the CT scans of four patients were selected during a consensus meeting with three experts as example scans for: no, mild, moderate, or severe brain atrophy. These CT scans were then used to score the severity of atrophy in the scans of the study patients.

Clinical information (diabetes, hypertension, cholesterol, history of ischemic heart disease, current smoking, and scores about neurological functioning) was collected on admission, and entered into the Maastricht Stroke Register (MSR), a prospective databank. All patients received standard stroke treatment and speech, physical, or occupational therapy as appropriate and when needed.

\section{Statistical Analysis}

Statistical analyses were performed with the statistical package for Social Sciences version 10 (SPSS-10). To perform valid analyses with sufficient power, we imputed data for some patients with missing data according to the method described in Tabachnick et al [51], by which an experienced clinician decided, blind for the outcome data, and based on available data and the patients' neuropsychological profile, how to impute the data. Data imputation was performed if there was at least one other test available for the same cognitive domain and if the missing test was administered at an earlier or later measurement time. Descriptive statistics were used to define the number of patients who did or did not recover from MCI. Differences between the two groups (reversible $\mathrm{MCI}$ versus stable $\mathrm{MCI}$ ) were assessed using $\chi^{2}$-test for categorical data and $\mathrm{T}$ - 
test analyses for continuous data. Moreover, odds ratios were defined to determine the predictability of demographic variables, baseline stroke risk factors, and CT variables for reversible MCI. In order to do this, age, level of education, and psychiatric complaints were dichotomized, because these variables were not linearly associated with the outcome variable, which is an assumption of logistic regression analysis. Age was dichotomized into 'old', 70 years and older and 'young', younger than 70, based on the median split. Educational level was dichotomized in 'low', primary education and vocational education or 'high', all other educational levels. Psychiatric complaints were dichotomized in 'high' when scores were higher than or equal to the scores of the norm-group, and 'low' when scores were lower than the scores of the norm-group. Multivariate logistic regression analyses were carried out to determine the independent relation between the variables that were univariately statistically significant, and reversible MCI.

\section{Results}

Hundred and eighteen patients were included in the study. Patient characteristics are described in table1.

\begin{tabular}{|c|c|c|}
\hline \multirow[b]{2}{*}{ Education low/high } & \multicolumn{2}{|c|}{ Data available $(\mathrm{N})$} \\
\hline & 118 & $68 / 50$ \\
\hline Sex M/F & 118 & $62 / 56$ \\
\hline Age mean (SD) & 118 & $67.7(11.9)$ \\
\hline MMSE mean (SD) & 118 & $25.8(2.8)$ \\
\hline Territorial/Lacunar/Hemorrhage & 117 & $45 / 64 / 8$ \\
\hline Side: Left/Right/Both & 113 & $47 / 65 / 1$ \\
\hline White matter lesions present & 112 & $25.0 \%$ \\
\hline Silent infarcts present & 112 & $30.6 \%$ \\
\hline Atrophy present & 104 & $68.3 \%$ \\
\hline
\end{tabular}

Thirty-nine $(33.1 \%)$ patients were classified as having reversible MCI, at some stage during the study. Twenty-four (20.3\%) patients were classified as having permanently reversible MCI. At 6 months after stroke, 117 patients were reassessed (1 patient was not assessed at 6 months after stroke, but assessed later), of whom $23(19.7 \%)$ were classified as having reversible MCI. At 12 months after stroke, 107 patients were re-assessed, of whom 14 (13.1\%) were classified as 
having reversible MCI. At 24 months after stroke, 100 patients were re-assessed, of whom $2(2.0 \%)$ were classified as having reversible MCI.

The 24 patients who recovered completely from MCI were compared with the patients who relapsed or who showed no recovery (Table 2). 


\begin{tabular}{|c|c|c|c|c|c|c|}
\hline & & Reversible & & & & \\
\hline & Data & $\mathrm{MCI}$ & $\mathrm{MCI}$ & & & \\
\hline & Available & $N=24$ & $N=94$ & & & \\
\hline & $(\mathrm{N})$ & $\mathrm{N}(\%)$ & $\mathrm{N}(\%)$ & OR & $95 \%$ CI & $\mathrm{p}$ \\
\hline Age & 118 & $68.9(11.6)$ & $67.4(11.9)$ & 1.2 & $.50-3.0$ & .65 \\
\hline $\begin{array}{l}\text { Education (Low/ } \\
\text { High) }\end{array}$ & 118 & $11 / 13$ & $39 / 55$ & 1.2 & $.48-2.9$ & .70 \\
\hline Sex (Female/Male) & 118 & $16 / 8$ & $40 / 54$ & 2.7 & $1.1-6.9$ & .04 * \\
\hline MMSE-score & 118 & $27.4(1.8)$ & $25.4(2.9)$ & 9.96 & $1.3-78.3$ & .00 * \\
\hline $\begin{array}{l}\text { Infarct/ } \\
\text { Hemorrhage }\end{array}$ & 117 & $22 / 2$ & $87 / 6$ & 1.3 & $.25-6.9$ & .75 \\
\hline Side:Left/Right & 112 & $11 / 12$ & $36 / 53$ & 1.6 & $.54-3.4$ & .52 \\
\hline $\begin{array}{l}\text { Stroke: Territorial/ } \\
\text { Lacunar }\end{array}$ & 109 & $10 / 12$ & $35 / 52$ & 1.2 & $.48-3.2$ & .66 \\
\hline $\begin{array}{l}\text { Asymptomatic } \\
\text { Present/Absent }\end{array}$ & 112 & $6 / 16$ & $28 / 62$ & .83 & $.29-2.3$ & .73 \\
\hline $\begin{array}{l}\text { White Matter } \\
\text { Lesions Present/ } \\
\text { Absent }\end{array}$ & 112 & $6 / 16$ & $22 / 68$ & 1.2 & $.40-3.3$ & .78 \\
\hline $\begin{array}{l}\text { Atrophy Present/ } \\
\text { Absent }\end{array}$ & 104 & $15 / 4$ & $56 / 29$ & 1.9 & $.59-6.4$ & .27 \\
\hline $\begin{array}{l}\text { Hypertension } \\
\text { Present/Absent }\end{array}$ & 114 & $7 / 17$ & $39 / 51$ & .54 & $.20-1.4$ & .21 \\
\hline $\begin{array}{l}\text { Diabetes Present/ } \\
\text { Absent }\end{array}$ & 116 & $4 / 20$ & $13 / 79$ & 1.2 & $.36-4.1$ & .75 \\
\hline $\begin{array}{l}\text { Hart disease } \\
\text { Present/Absent }\end{array}$ & 116 & $2 / 22$ & $14 / 78$ & .51 & $.11-2.4$ & .76 \\
\hline $\begin{array}{l}\text { Cholesterol } \\
\text { Present/Absent }\end{array}$ & 84 & $4 / 13$ & $12 / 55$ & 1.4 & $.39-5.1$ & .59 \\
\hline $\begin{array}{l}\text { Current smoking } \\
\text { Present/Absent }\end{array}$ & 118 & $4 / 20$ & $35 / 59$ & 2.9 & $.94-9.5$ & .06 \\
\hline $\begin{array}{l}\text { Therapy } \ddagger \\
\text { Present/Absent }\end{array}$ & 118 & $16 / 8$ & $58 / 36$ & 1.2 & $.48-3.2$ & .65 \\
\hline $\begin{array}{l}\text { Score SCL-90 } \\
\text { (mean, SD) }\end{array}$ & 118 & $130.1(66.4)$ & $125.1(69.6)$ & 1.5 & $.56-3.9$ & .43 \\
\hline
\end{tabular}


Patients with permanently reversible MCI had a higher MMSE score at baseline and were more often females. Even after correction for multiple comparisons $(0.05 / 17=.00291)$, baseline MMSE score still differentiated between the groups. The other variables were not significantly different between the two groups.

Multiple logistic regression analyses were performed with the variables, that were significant in the univariate analyses. In the multivariate analysis, baseline MMSE and female sex were independently related with recovery from MCI. Patients with higher MMSE scores at baseline had almost 10 times higher chance of recovery $(\mathrm{OR}=9.96 ; \mathrm{CI}=1.26-78.29, \mathrm{p}=.00)$, women had almost a 3 times higher chance of recovery $(\mathrm{OR}=2.75, \mathrm{CI}=1.1-6.9, \mathrm{p}=.04)$.

\section{Discussion}

Our results suggest that long-term recovery from MCI after stroke does indeed take place. About $20 \%$ of the patients who had cognitive deficits 1 month after stroke showed normal cognitive functioning at a later assessment. In most patients recovery occurred between 1 and 6 months after stroke, which is in line with the study of Tham et al., who also found that after 1 year $10 \%$ of the stroke patients with cognitive impairment (but no dementia) had recovered [113]. The percentage of patients showing this improvement was lower in a populationbased study (2.7\% after 5 years) [15]. MCI-patients without cerebrovascular disease also showed recovery [117-122, 126]. Thus, although MCI is generally studied in the context of progression to dementia, recovery from $\mathrm{MCI}$ is possible. It is important to identify patients who may recover from MCI because the pessimistic view that cognition will only get worse is not applicable to all patients with MCI. We found that a higher MMSE score at baseline and female sex were predictors for recovery from $\mathrm{MCI}$ after stroke, as did an earlier study with regard to baseline MMSE score [113]. Thus, people who are classified as cognitively impaired but who have a high baseline cognitive performance have a greater chance of recovery from MCI. Although post-hoc analysis did not reveal significant effects, the presence of risk factors for stroke was lower in women than in men (29.1\% versus $34.4 \%)$ and in patients with high baseline MMSE scores, than in those with low baseline MMSE scores (28.4\% versus $42.9 \%)$.

Other studies reported that right hemisphere infarcts and lower level of consciousness at baseline predicted recovery 2 years after stroke [123], whereas others found that left hemisphere infarcts and absence of diabetes were associated with improvement [89]. The reason why we did not find CT-variables or stroke risk factors to be associated with recovery from $\mathrm{MCI}$ is probably because we included only patients who had a relatively good cognitive performance at baseline (MMSE $\geq 15$ ), and the excluded patients with MMSE < 15 would probably have had less chance of cognitive recovery. If we had included these patients, 
the difference between those who recovered and those who did not would have been greater. Even though our results are limited to patients with relatively mild impairments, this patient group is clinically relevant, because these patients are more likely to be discharged and sent home to care for themselves, but nevertheless may still encounter problems in resuming their daily activities.

Although we could not verify our hypotheses that the patients with cognitive recovery would have less brain damage or fewer stroke risk factors, we found that patients who recovered had silent infarcts on CT, hypertension, and ischemic heart disease, less often than the patients who did not recover from post-stroke MCI, but the differences were not statistically significant. They also smoked less, and had received paramedical therapy more often. Although earlier studies showed risk factors for stroke to be associated with decreased cognitive functioning $[71,127,128]$ we could not reproduce this finding, which might have been due to a lack of power.

Cognitive deficits in patients with MCI could be related to depressive symptoms; however, we did not find differences in total SCL-90 score or scores for the subscale 'depression' between the two groups.

Our study had some limitations. First, the patients were tested four times. Although a learning effect could not be ruled out, this effect is minimal for the neuropsychological tests used in this study. Moreover, we used parallel versions of the tests whenever possible [93]. Secondly, MCI was diagnosed on the basis of both clinical and psychometric definitions; however, we did not include memory complaints as diagnostic criterion, in contrast to the Mayo Clinic criteria [81]. Busse et al found that the prevalence of MCI increased when subjective cognitive complaints were excluded from the MCI criteria, and thus we may have overestimated the number of patients diagnosed with MCI [108]. However, this probably does not affect our main conclusions, as the focus of our study was to find variables that predicted recovery.

In conclusion, our results suggest that about $20 \%$ of patients with MCI recover and that a higher MMSE-score at baseline and female sex are independent predictors of this recovery. 


\section{Demographic and CT-scan features} related to cognitive impairment in the first year after stroke

\footnotetext{
Adapted from

Rasquin SMC, Verhey FRJ, Oostenbrugge van RJ, Lousberg R, Lodder J. Demographic and CT-scan features related to cognitive impairment in the first year after stroke. Journal of Neurology, Neurosurgery, and Psychiatry. Journal of Neurology, Neurosurgery, and Psychiatry, 2004. 75:1562-1567.
} 


\section{Abstract}

Background: The aim was to study features (CT-scan and demographic) that could be related to vascular cognitive impairment at 1, 6, and 12 months after stroke.

Methods: One hundred seventy-six patients with a first-ever brain-infarct, an MMSE $\geq 15$, age older than 40 years, and without pre-stroke dementia, and other neurological or psychiatric disorders participated. The following CT-features were recorded: side of infarct; lacunar or territorial infarct; white matter lesions; silent infarcts; and brain atrophy. Demographic features were: age, level of education, and sex. Three groups of patients with dementia, vascular cognitive impairment (VCI), or vascular mild cognitive impairment (MCI) were compared with patients without cognitive disorders.

Results: At 1 month none of the variables were predictors of dementia; at 6 months older age $(\mathrm{OR}=9.4)$, low education $(\mathrm{OR}=14.7)$, and territorial infarct $(\mathrm{OR}=10.6)$ predicted dementia; and at 12 months low education $(\mathrm{OR}=8.7)$ and prestroke cerebrovascular damage $(\mathrm{OR}=7.4)$ predicted dementia. Predictors of VCI were low education ( $\mathrm{OR}=3.4$ ) and territorial infarct $(\mathrm{OR}=2.4$ ) at 1 month; older age $(\mathrm{OR}=4.3)$ and low education $(\mathrm{OR}=4.1)$ at 6 months; and older age $(\mathrm{OR}=3.5)$ at 12 months. Predictors of vascular MCI were low education $(\mathrm{OR}=$ $4.9)$ and territorial infarct $(\mathrm{OR}=3.6)$ at 1 month; and older age and lower education at 6 months $(\mathrm{OR}=3.4$ and $\mathrm{OR}=3.7$ respectively) and at 12 months $(\mathrm{OR}=3.5$ and $\mathrm{OR}=2.28$ respectively).

Conclusions: Territorial infarct, older age, and low educational level, are predictors of cognitive disorders after stroke. 


\section{Introduction}

The risk of dementia and other cognitive disorders increases after stroke. Stroke contributes to the development of cognitive disorders in nearly $20 \%$ of elderly patients $[12,129]$. Stroke-related features such as multiple strokes, white matter lesions, left hemisphere infarct location, atrophy, and volume of infarcted tissue are associated with an increased risk of post-stroke dementia [16, 17, 86, 130135]. Age and level of education have also been reported as risk factors for vascular dementia (VaD) $[17,62,86,133]$. Traditionally, patients with VaD have been compared with non-demented stroke patients in order to detect associations between stroke-related factors and cognitive disorders (usually dementia). As such cognitive disorders less severe than dementia were left out of consideration. However, milder cognitive disorders are, more likely than $\mathrm{VaD}$ to occur, after stroke $[18,29,56,77,88]$. These milder deficits, also called vascular mild cognitive impairment (MCI) or vascular cognitive impairment no dementia (CIND), may be barely perceptible in an early stage but may carry the risk of progression to more severe loss of cognitive functions [10, 79, 136]. If vascular $\mathrm{MCI}$ is as a transition phase in the development of dementia it is important to identify specific risk factors for vascular $\mathrm{MCI}$, in order to attempt to prevent the progressive decline in cognitive function $[109,137]$. The few studies that investigated risk factors for cognitive decline in patients with either vascular MCI or $\mathrm{VaD}$ found older age, educational level, volume of infarcted tissue, and left hemispheric infarct location to be associated with cognitive impairment after stroke $[18,59,90,138,139]$. However these studies did not differentiate between patients with dementia and patients with less severe cognitive disorders. Consequently, mechanisms related to post-stroke cognitive disorders without dementia have not been identified. In this study, we distinguished between patients with dementia and those with cognitive disorders without dementia (vascular MCI). As such, homogeneous groups, based on cognitive functioning arise and generalisations about risk factors for vascular cognitive impairment can be made for subgroups. Because little is known about the influence of stroke-related risk factors on the development of cognitive impairment over time, we also monitored the cognitive abilities of our sample over 1 year. Our aim was to identify post-stroke predictors (stroke-related and demographic) of VCI (with or without dementia) at 1, 6, and 12 months after stroke, with special emphasis on brain-abnormalities as visualized by computed tomography (CT). 


\section{Methods}

\section{Patients}

The Maastricht CODAS (COgnitive Disorders After Stroke) project is a prospective, observational study investigating the development of cognitive impairment after stroke, and to identify factors related to these disorders.

The study includes patients with a first-ever cerebral infarct who are older than 40 years (to exclude atypical stroke), have adequate post-stroke fluency in Dutch, and who have an initial Mini Mental State Examination (MMSE) $\geq 15$ (to ensure neuropsychological testing is possible). Exclusion criteria are severe aphasia and other neurological or major psychiatric disorders, which could lead to cognitive impairment other than the qualifying event. For this report patients with pre-stroke dementia were also excluded.

Consecutive stroke patients admitted to the University Hospital Maastricht or who visited the outpatient neurological clinic between January 2000 and July 2001 entered the study. During the inclusion period, 592 patients came to the hospital with a stroke. Of these 592 patients 396 were excluded (46 MMSE < 15, 20 had other neurological or psychiatric disorders, 5 were not native Dutch speaking, 9 were younger than 40 years, 57 had a stroke located in the brainstem or cerebellum, 89 were not first-ever strokes, 80 died within 1 month after stroke, 9 lived too far from the hospital, 6 were admitted too long after their stroke, 35 refused to participate, 6 were in a coma, and 34 had severe aphasia). Hundred and ninety-six were eligible for the CODAS study. Of these 196 participants 18 had a hemorrhagic stroke and 2 patients had dementia before stroke and were consequently excluded.

Stroke was diagnosed by an experienced neurologist according to standardized, well-accepted criteria described elsewhere [53]. All patients had at least one brain CT scan. In most patients, CT was performed at admission or 1 day later ( $\mathrm{N}=93,52.9 \%)$, the median was 1 day with a range of 0 to 150 days. If patients had a second scan on which lesions were seen more clearly, this scan was used. Patient data were entered into the Maastricht Stroke register (MSR), a prospective databank containing clinical information on all stroke patients treated in the University Hospital Maastricht [53]. Data on patient age ('high', 70 years and older, or 'low', younger than 70, based on the median split), sex, and level of education ('low', primary education and vocational education or 'high', all other educational levels). All patients were tested neuropsychologically within 1 month (mean 1.07 months, SD 0.34) of stroke, and again at 6 months (mean 6.06, SD 0.41) and 12 months (mean 12.08, SD 0.36). CT scanning was not repeated at follow-up. 
To define whether patients had a cognitive disorder, they were compared with a control group from the MAastricht Aging Study (MAAS) [49]. This population-based study focuses on determinants of normal cognitive aging and covers the full adult life-span of initially healthy individuals. Normative data from this study were stratified according to age, sex, and educational level. Impairment was defined as a score below the 10th percentile of the control group from MAAS; this cut-off is in line with clinical practice.

\section{Computed Tomography}

The following features were recorded: side of the symptomatic infarct (left or right hemisphere); stroke type (lacunar or territorial infarct); presence of white matter lesions; presence of silent infarcts; and brain atrophy. For definitions we refer to de Jong et al. [53]. White matter lesions (WML) were defined as focal or diffuse hypodensities in the periventricular or deep white matter, not involving the cortex, with ill-defined margins to differentiate them from infarction [54]. The presence of WML around frontal or occipital ventricular horns or in the centrum semi ovale was rated separately. No effort was made to quantify the degree of WML density in these different sites, as this might depend on the tuning of the scanning and a certain variability related to the procedure of making the scan hard-copy. Silent brain infarction was defined as a low-density area on the CTscan, compatible with infarction but without a history of stroke as taken from the patient's history, from family, or any other accessible information. Also, the stroke-symptoms at study entry had to be anatomically incompatible with such silent infarcts. Old infarcts can usually be distinguished from new ones because they are more hypodense, and there may be signs of surrounding tissue loss like retraction of brain structures towards the infarct. The degree of atrophy was scored in a semi-quantitative way (none, mild, moderate, and severe), according to the criteria of Leys et al [55]. In short, CT-scans of four patients were selected during a consensus-meeting by three experts as example scans for the four categories: no, mild, moderate, or severe brain atrophy. CT scans of study patients were graded into one of these categories by visual comparison with the example scans. Silent infarcts or white matter lesions, or both were defined as 'prestroke cerebrovascular damage'. Two neurologists examined the CT scans independently. They were unaware of the neurological signs and the neuropsychological data. In case of difference, consensus was reached by discussion. Inter-rater agreement (consensus reached by discussion was left out) in the classification of patients by stroke features on CT was excellent, with a $\mathrm{K}$ of 0.88 for symptomatic side, $\mathrm{k}$ of 0.85 for type of stroke, $\mathrm{k}$ of 0.60 for white matter, $\mathrm{k}$ of 0.79 for silent infarcts, and $\mathrm{k}$ of 0.69 for atrophy. 


\section{Patientgroups}

Cognitive functioning was assessed using a neuropsychological test battery described elsewhere [29]. Memory, mental speed, interference susceptibility, orientation, language, praxis, and abstract thinking were assessed based on the criteria of Roman et al [24] .

'Dementia' was diagnosed independently by a neuropsychiatrist and a neuropsychologist according to the DSM IV criteria, based on all available data such as a questionnaire about daily functioning [38], clinical information from a neuropsychologist, and cognitive profile. In 5 patients there was a disagreement on the final diagnosis and these patients were classified as not demented. 'Vascular $\mathrm{MCI}^{\prime}$ was diagnosed when patients had at least one cognitive deficit. Our definition of MCI is somewhat broader than the traditional criteria of Petersen et al., which focused on amnestic disorders. We used their amplified criteria [80, 81]. The group 'Vascular Cognitive Impairment' (VCI) contained both patients with dementia or patients with vascular MCI. Patients without cognitive disorders were assigned to the 'No Cognitive Disorder' group. At each measurement, patients were re-evaluated for cognitive deficits and re-assigned to one the four groups (dementia, $\mathrm{VCI}$, vascular MCI, or no cognitive disorder) independent of the classification at other assessments.

\section{Statistical Analysis}

Statistical analyses were performed using SPSS version 10 .

Demographic and CT features, were compared in three different models:

I Patients with dementia versus patients without cognitive deficits; II Patients with VCI versus patients without cognitive deficits; and III Patients with vascular MCI versus patients without cognitive deficits.

For categorical variables $\chi^{2}$ and Fisher's exact test were used; a two-sample Students' t-test was used for continuous variables. Logistic regression analyses were conducted to determine the predictability of the $\mathrm{CT}$ and demographic variables of dementia, $\mathrm{VCI}$, and vascular MCI. Variables which distinguished ( $\mathrm{p}$ $<0.05$; two-tailed) between the groups in the univariate analyses were entered in a multiple logistic regression model. If either white matter lesions or silent infarcts significantly predicted the outcome group, we entered 'prestroke cerebrovascular damage' into the multiple logistic regression model.

Missing data were imputed according to a standard procedure as described in Tabachnick et al. [51]. Data imputation was performed if there was at least one other test available within a specific cognitive domain and if the missing test was administered on an earlier or later measurement period. 


\section{Results}

One hundred and seventy-six patients were examined 1 month after stroke: 101 men $(57.3 \%)$ and 75 women $(43.7 \%)$, with an average age of $67.9(\mathrm{SD}=12.5)$ years (Table 1). Of the included patients, 100 patients (56.8\%) had a low educational level. Mean MMSE score was 25.5 (SD = 3.5). Ninety patients $(50.5 \%)$ had no symptomatic ischemic lesion on CT. At the moment of CT-examination, the CT scans of 11 patients $(6.2 \%)$ could not be retrieved from the radiological department archives. Clinical data were used to categorize these patients and those without a CT, as having lacunar or territorial brain infarction (67 were classified as lacunar, 34 as territorial). Ninety-nine patients (56.3\%) had lacunar stroke and $77(43.8 \%)$ had territorial stroke. Ninety-six (53.9\%) patients had a stroke in the right hemisphere, $78(44.3 \%)$ in the left hemisphere, and $2(1.2 \%)$ patients in both hemispheres. Thirty-six (20.5\%) patients had white matter lesions, 110 (62.5\%) had atrophy, and 57 patients (32.4\%) had at least one silent infarct.

At 6 months after stroke, 4 (2.3\%) patients had died and $13(7.4 \%)$ refused further participation, 4 refused temporally $(2.3 \%)$ and 1 patient could not be traced anymore (0.6\%). At 12 months after stroke, 5 (3.2\%) patients had died and $9(5.8 \%)$ refused participation. Patients not completing the study did not differ from those who remained in the study with respect to baseline MMSE score, age, educational level, and sex.

Table 1 shows the distribution of the demographic and clinical variables at baseline for the four groups.

Table 1: Demographic and clinical variables for the four groups at baseline

\begin{tabular}{lllll}
\hline & & & & No Cognitive \\
& Dementia & VCI & Vascular MCI & Disorder \\
\hline $\mathrm{N}$ & 17 & 142 & 125 & 34 \\
Age (mean, SD) & $78.2(6.6)$ & $69.0(12.3)$ & $67.7(12.4)$ & $63.6(12.8)^{*}$ \\
Sex (M-F) & $9-8$ & $78-64$ & $69-56$ & $23-11$ \\
Education (L-H) & $10-7$ & $89-53$ & $79-46$ & $11-23^{*}$ \\
MMSE (mean, SD) & $19.8(2.3)$ & $24.8(3.5)$ & $25.5(3.1)$ & $28.3(1.5)^{*}$ \\
Hypertension (pr-ab) & $10-5$ & $79-43$ & $89-38$ & $18-13$ \\
Diabetes (pr-ab) & $13-2$ & $104-20$ & $91-18$ & $30-3$ \\
Cholesterol (pr-ab) & $8-0$ & $70-19$ & $62-19$ & $25-3$ \\
Hartfailure(pr-ab) & $10-4$ & $104-20$ & $94-16$ & $29-4$ \\
Current smoking (pr-ab) & $6-5$ & $52-43$ & $46-38$ & $18-10$ \\
\hline
\end{tabular}

M: Male | F: Female | L: Low | H: High | pr: presence | ab: absence |

MCI: Mild Cognitive Impairment | VCI: vascular Cognitive Impairment $\mid{ }^{*} p<.05$ 
At 6 months after stroke 13 patients were demented, 99 were diagnosed as vascular MCI and 42 did not had any cognitive deficits. At 12 months after stroke 14 patients were demented, 85 were diagnosed as vascular MCI and 45 did not had any cognitive deficits. At 6 months after stroke 3 patients were progressed from 'vascular $\mathrm{MCI}^{\prime}$ to 'dementia', and 9 patients progressed from 'no cognitive disorder' to 'vascular MCI'. At 12 months after stroke, 3 patients progressed from 'vascular $\mathrm{MCI}^{\prime}$ to 'dementia' and 7 patients progressed from 'no cognitive impairment' to 'vascular $\mathrm{MCI}^{\prime}$. Patients who deteriorated were more often of female sex $\left(x^{2}=4.5, \mathrm{df}=1, \mathrm{p}=.03\right)$. The other variables (age, level of education, stroke type, side of the infarct, white matter lesions, asymptomatic infarcts, and atrophy) did not differentiate between the patients who deteriorated and those who did not.

Older age, territorial infarct, silent infarcts, prestroke cerebrovascular damage, and atrophy were associated with dementia 1 month after stroke (Table 2). At 6 months, older age, lower educational level, territorial infarct, and prestroke cerebrovascular damage were associated with dementia. At 12 months, the same variables plus silent infarcts were associated with dementia (Table 2).

At 1 month after stroke, lower educational level and territorial infarcts were associated with VCI; at 6 months older age, lower educational level, territorial infarct, and atrophy were associated with VCI; and at 12 months older age, female sex, lower educational level, white matter lesions, prestroke cerebrovascular damage, and atrophy were associated with VCI (Table 3).

At 1 month after stroke, lower educational level and territorial infarcts were associated with vascular MCI; at 6 months older age, lower educational level, and territorial infarct were associated with vascular MCI; and at 12 months older age, female sex, lower educational level, white matter lesions, and prestroke cerebrovascular damage were associated with vascular MCI (Table 4). In all univariate analyses, age was also graded in other categories ( $60 \mathrm{vs}<60 ; 70$ vs $<60,80$ vs $<60)$. This did not change the associations.

Since the number of regression analyses was quit large ( 9 variables, 3 measurements periods and 3 groups, resulting in 81 analyses), it could be argued to correct the significance level to prevent type-one errors. However, since the adjusted p-value would become very small (padj. < 0.0006), the probability to find a significant effect at all was reduced to an extremely low level. Because of the explorative character of the present study, it was decided not to adjust the p-level, but to interpret the significance of the statistical tests with care, as well as to judge whether the observed effects were clinically relevant.

Multivariate analyses using logistic regression were performed to identify factors that were independently associated with cognitive impairment after 
stroke (Table 5). In model I ('dementia' versus 'no cognitive disorder' at 1 month after stroke), age, location (territorial or lacunar) infarct, prestroke cerebrovascular damage, and atrophy were included as variables. No independent predictors of dementia could be identified. Older age, lower educational level, and territorial infarct location were independently associated with dementia at 6 months after stroke. Lower educational level and prestroke cerebrovascular damage were independently correlated with dementia at 12 months after stroke.

In model II ('VCI' versus 'no cognitive disorder') lower educational level and territorial infarct were independently correlated with VCI at 1 month after stroke. Both older age and lower educational level were independently correlated with $\mathrm{VCI}$ at 6 months after stroke. Older age was independently correlated with $\mathrm{VCI}$ at 12 months after stroke. In model III ('vascular $\mathrm{MCI}^{\prime}$ versus 'no cognitive disorder'), lower educational level and territorial infarct location were independently correlated with vascular $\mathrm{MCI}$ at 1 month after stroke. Both older age and lower educational level were independently correlated with vascular MCI at 6 and 12 months after stroke. 


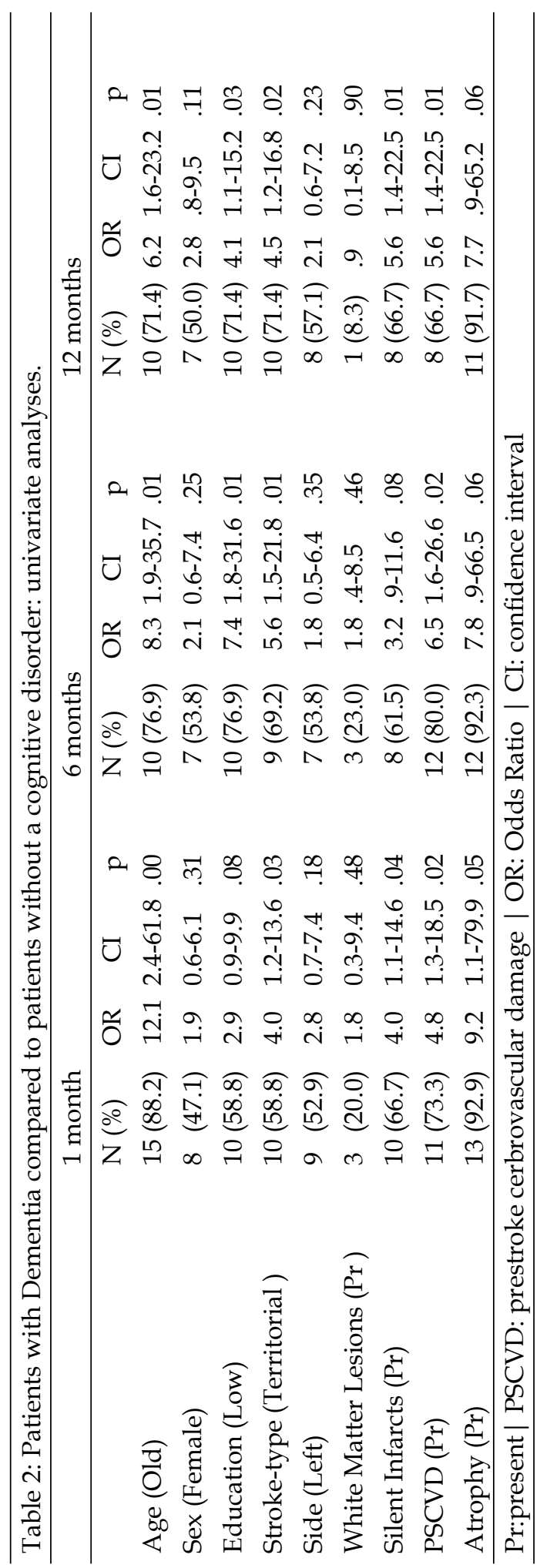




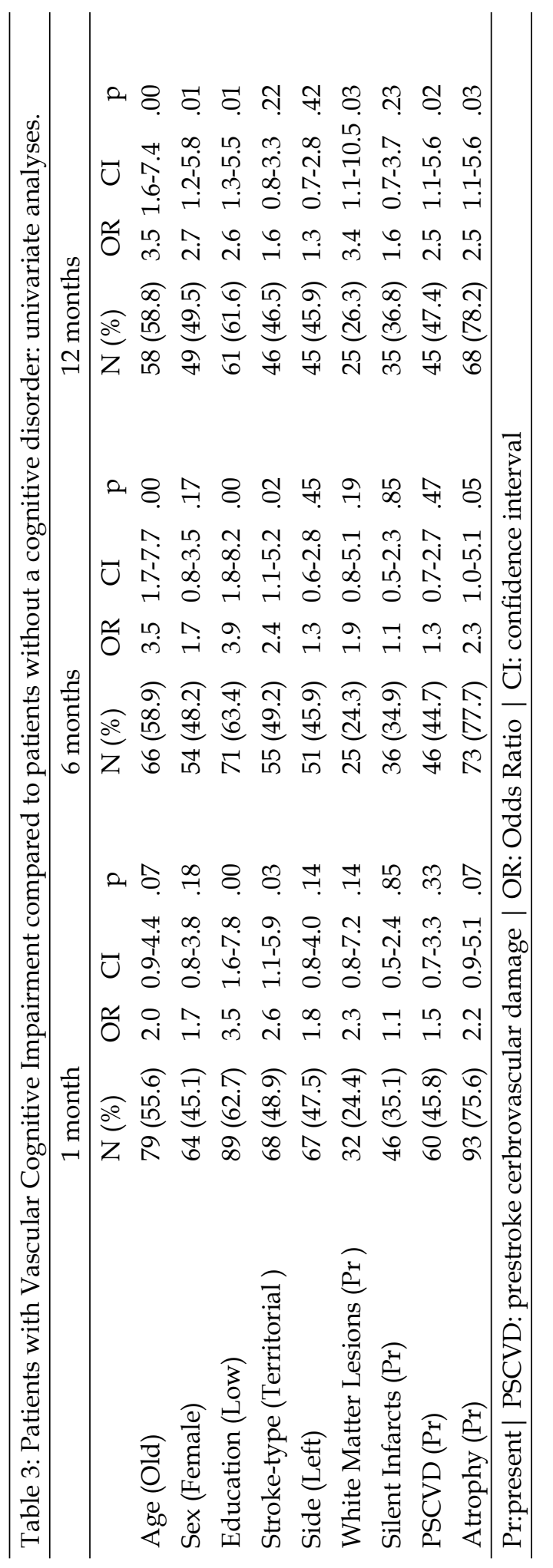




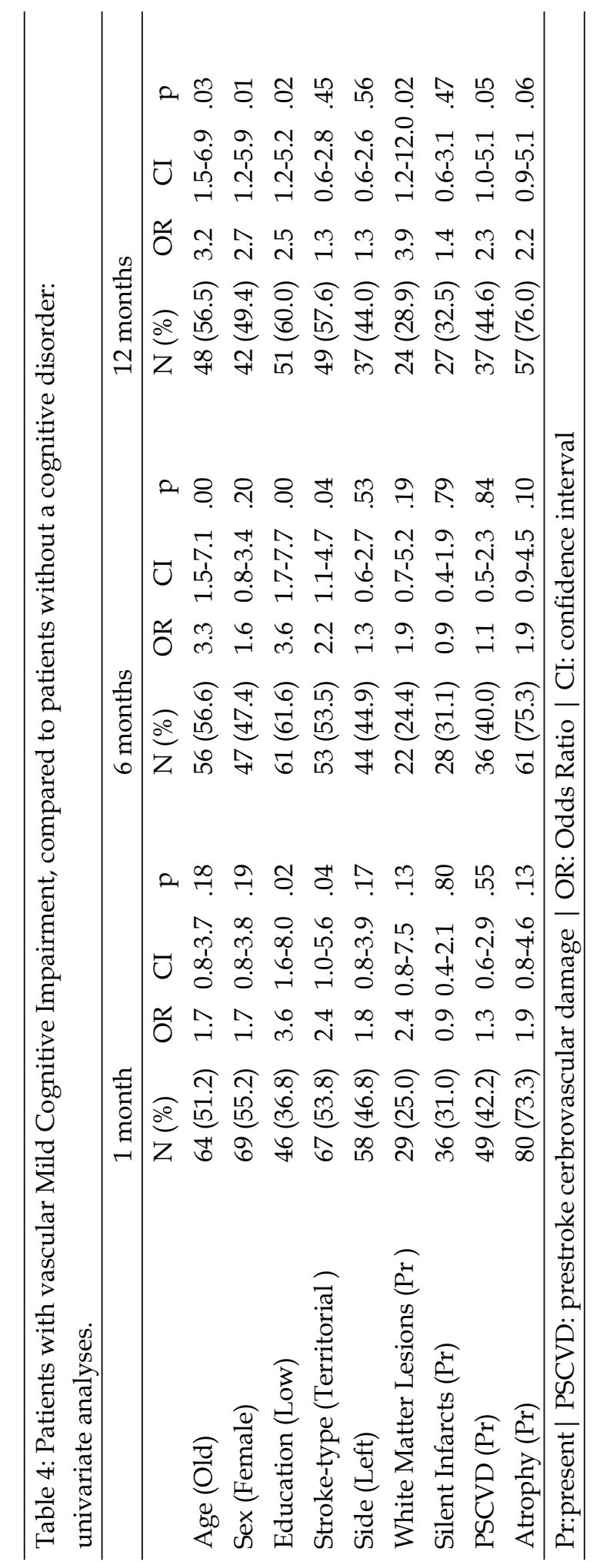




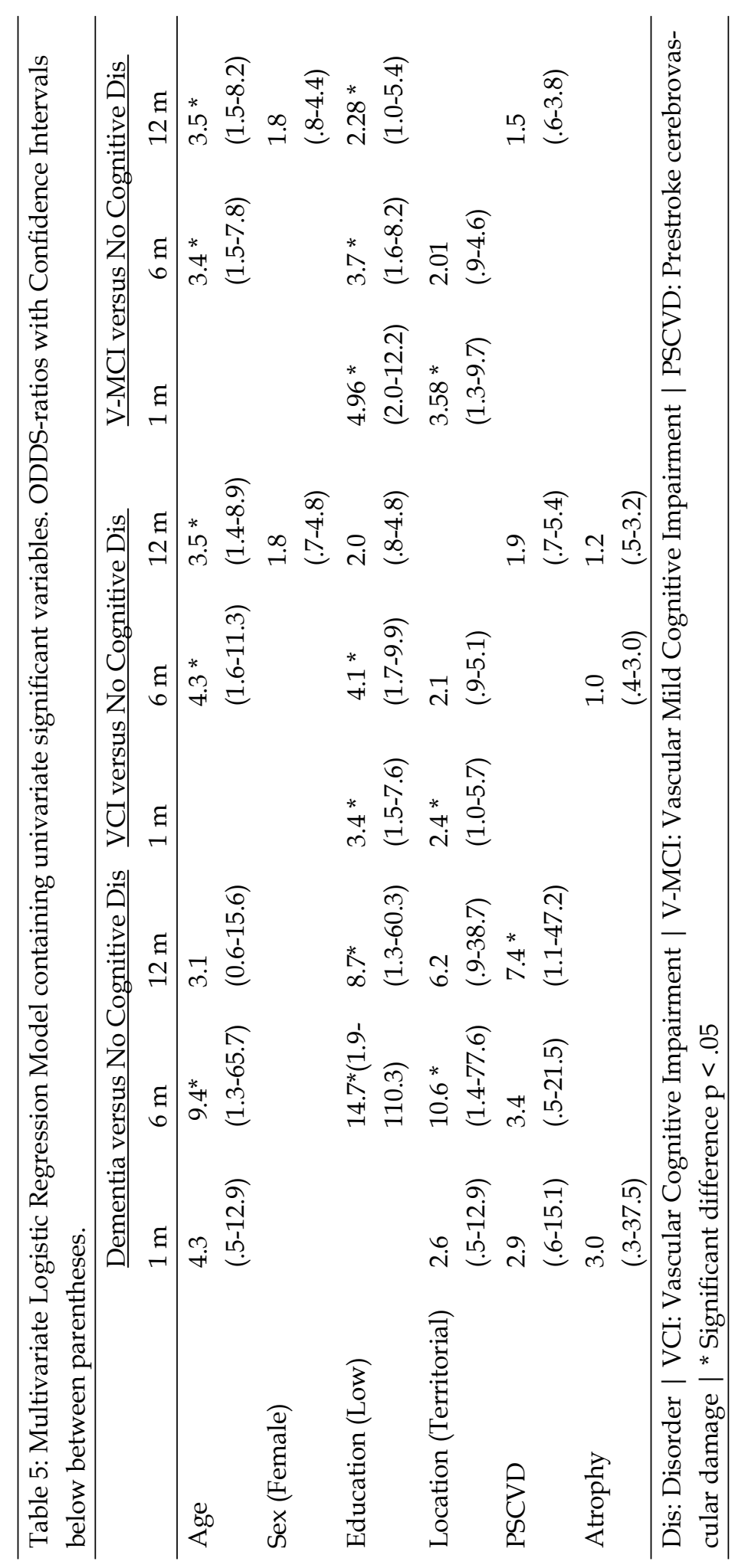




\section{Discussion}

We investigated specific characteristics (stroke-related and demographic) that could be related to dementia, $\mathrm{VCI}$, and vascular MCI at 1, 6, and 12 months after stroke, with special emphasis on neuroimaging data. Older age and lower educational level were found to be, in all groups, important predictors, after stroke. Moreover, territorial as opposed to lacunar stroke was also a predictor1 month after stroke.

Other studies of post-stroke dementia have reported similar risk factors [17, 62, $86,133,134]$, however, these studies identified several stroke-related risk factors for dementia, whereas we identified only territorial infarction. Territorial infarction might be associated with cognitive impairment because of the importance of these regions for higher cerebral cognitive functioning [16, 90, 140]. Most studies found white matter lesions and atrophy to be independent contributors to post-stroke dementia [17, 131, 134, 141-144], whereas we did not. One reason for this discrepancy is that we used CT instead of magnetic reasonance imaging (MRI) which is more sensitive. However, in most hospitals patients with stroke have a CT on admission, and as such the data presented here are of clinical relevance. An other reason is that few patients had white matter lesions $(21 \%)$ in our study whereas in other studies, more than half of the patients had such lesions $[131,133,142]$. Our low number of patients with white matter lesions could be a consequence of the exclusion of patients with MMSE $<15$. It is quite possible that some of these excluded patients had insidiously progres-sing cognitive decline before stroke, which is related to white matter lesions $[86,145,146]$. Thus our exclusion of patients with severe cognitive impairments may have resulted in the exclusion of patients with white matter lesions.

The studies reported above examined dementia after stroke, and as such they neglected patients who did not meet criteria for dementia but who nevertheless might have had cognitive impairments. Other investigators have investigated the association between cognitive disorders, not restricted to dementia, and stroke-related features, but few included multivariate analyses in the study design [18, 59, 90, 138, 139]. Schmidt et al. [90] found multiple infarcts and temporal lobe infarction to be risk factors for cognitive disorders. Patel et al. [139] reported left hemisphere infarction, older age, ethnicity, socio-economic status, and stroke severity to be important contributors to the development of cognitive disorders after stroke. The only stroke-related factor that contributed independently to $\mathrm{VCI}$ early after stroke in our study was territorial infarction. Unlike other stu-dies we were able to diagnose more subtle cognitive deficits because of the use of an extensive and sensitive set of neuropsychological tests. Patients with only subtle cognitive deficits have either less brain damage or they might have brain damage in regions that are of less importance for cognitive 
functioning. This could explain why we found fewer associations between CTfeatures and cognitive disorders.

Although recent research has focused on post-stroke cognitive impairment not restricted to dementia, to our knowledge no studies have reported about cognitive disorders without dementia. We found that early after stroke, territorial infarction, older age, and lower educational level, were predictors of vascular $\mathrm{MCI}$. The univariate analyses revealed that pre-existing vascular brain damage (white matter lesions or silent infarcts) became more important at later times after stroke. This could indicate that with increasing time after the stroke the influence of the initial infarct decreases, and the influence of pre-existing brain damage becomes more important. In most stroke patients white matter lesions or silent infarcts progress over time, despite standard treatment, with the consequence that cognitive functioning may decline [54, 92, 147, 148]. We did not investigate whether the patients with cognitive decline had progressively increasing brain damage at 6 and 12 months, but in the future we plan to investigate the relation between accumulating brain damage and cognitive decline.

There are certain limitations to this study. First, only one third of the total stroke population admitted to the hospital during the inclusion period was included in the study. However, our series was not affected by referral bias, because the University Hospital Maastricht is the only hospital in the region. Second, to perform valid analyses with sufficient power we imputed data for some patients with missing data. This was done by an experienced neuropsychologist who decided, based on available data and the patients' neuropsychological profile, how to impute the data. Although this method is subjective, comparison of this method with one based on regression analyses did not yield different results. We also compared for all analyses the database with the imputed data with the database without the imputed data and all conclusions remained the same. Third, because we excluded patients with MMSE $<15$ we may have excluded patients with specific stroke-related features such as territorial infarction or white matter lesions. However, we studied cognitive decline in those patients for whom such decline might be most relevant, namely patients without any prior severe cognitive problems. Fourth, infarct site and size may be relevant to study as independent predictors for post-stroke cognitive impairment. However, the fact that only about one half of the patients had an infarct visible on $\mathrm{CT}$, which was mainly due to early scanning, forestalled such analysis. Another study also found in $50 \%$ of the patients, visible lesions on CT within 48 hour after stroke [149]. Fifth, in our study design it could not be ruled out that some patients with dementia might have had mixed type instead of vascular type. Although WML are generally held to be caused by vascular insufficiently, they can also occur in patients with dementia of the Alzheimer's type ([150] . However, by excluding patients with MMSE < 15 and patients with dementia before stroke, we made the likelihood of including such patients low. Despite some limitations, this study is the 
first to investigate the relation between early CT features and post-stroke cognitive impairment on a longitudinal basis. Territorial infarction, older age, and lower educational level are important risk factors for cognitive disorders after stroke. Pre-stroke damage as diagnosed by CT, are probably related to cognitive disorders later after stroke. 


\section{$X$}

\section{Progressive brain damage in relation to cognitive performance after first-ever stroke: a 2-year follow-up study}

\section{Adapted from}

Rasquin SMC, Verhey FRJ, Lousberg R, Lodder J. Progressive brain damage in relation to cognitive performance after first-ever stroke: a 2-year follow-up study. Journal of Neurology, Neurosurgery, and Psychiatry. Revised version submitted. 


\section{Abstract}

Background: Stroke is one of the most common causes of cognitive impairment in the elderly. Brain damage after stroke progresses in a substantial number of the patients. The aim of this study was to investigate whether progressive brain damage is associated with cognitive functioning after first-ever stroke.

Methods: One-hundred and one stroke patients were followed up for 2 years. Neuropsychological functioning was assessed at 1, 6, 12, and 24 months after stroke. CT was performed on all patients at baseline and 2 years after stroke. Progression in white matter lesions and (silent) infarcts was recorded.

Results: Patients with progressive brain damage performed worse on cognitive tasks, both 1 and 24 months after stroke, yet changes in cognitive functioning was not different from that of patients without progressive brain damage. During the follow-up, on most cognitive domains improvement was noticed. Conclusions: Although patients with progressive brain damage after a first stroke performed somewhat worse on cognitive tests than those without such damage, both groups showed an improved or stable performance 2 years later. Thus, there is not a simple relation between progression of ischemic brain damage and decline in cognitive functioning after first-ever stroke. 


\section{Introduction}

White matter lesions (WMLs) and silent infarcts are frequently seen on brain images recorded from patients after they have had a stroke. Despite standard treatment for secondary stroke prevention, this pre-existent brain damage progresses over time. More than $25 \%$ of patients with a first-ever ischemic stroke show progression of WMLs or an increase in the number of silent infarcts on computed tomography (CT ) 3 years later[54]. Although this increase in brain damage is suggested to be associated with cognitive decline [74, 151-154], there has been little research on the relation between an increase in 'silent' ischemic lesions and cognitive functioning over time. Most studies on cognitive functioning after stroke have focused on the development of dementia $[2,6,155]$. One study found a relation between increased brain damage and cognitive decline after stroke in patients with Vascular Dementia (VaD) [156]. However, the concept VaD has been replaced by the broader concept of Vascular Cognitive Impairment (VCI) because $\mathrm{VaD}$ suggests that dementia is caused by a single mechanism and has consistent manifestations, which is not the case [7].

The VCI concept allows studies on cognitive functioning after stroke to focus on the more subtle forms of cerebrovascular disease as cause of cognitive impairment [157]. In an earlier study, we found that the association between pre-existing brain damage (WMLs and silent infarcts), measured directly after stroke, and VCI became stronger at later times after the stroke [100]. This finding, combined with the knowledge that pre-existent brain damage increases after stroke [54], led us to hypothesize that progression of WMLs, silent infarcts, or both is related to cognitive decline following stroke. In this study we measured cognitive functioning early after first ever stroke and 2 years later.

\section{Methods}

\section{Patients}

Patients were collected from the CODAS study (Cognitive Disorders After Stroke), a longitudinal, prospective study aimed at identifying cognitive disorders and their risk factors during the first 2 years after stroke [29, 101]. Patients were tested neuropsychologically at 1, 6, 12, and 24 months after stroke. For this report the data of the 1 and 24-month assessments were used. Consecutive patients who were admitted to hospital or who visited the outpatient clinic of the University Hospital Maastricht between January 2000 and August 2001 because of a stroke were asked to participate in this study. An experienced 
neurologist diagnosed stroke, based on clinical data supported by CT findings. Clinical information was collected on admission and entered into the Maastricht Stroke Register (MSR). This prospective databank contains information about the presence of diabetes, hypertension, cholesterol, ischemic heart disease, family history of vascular disease, scores about neurological functioning, and final stroke subtype diagnosis for all stroke patients.

Inclusion criteria for this study were: first-ever hemispheric stroke, MMSE score $\geq 15$ (to ensure valid testing), and age older than 40 years. Exclusion criteria were: prestroke dementia, no baseline $\mathrm{CT}$, neurological disorder other than the qualifying event, or major psychiatric disorder which could lead to cognitive deficits. One hundred and eighty-nine patients fulfilled these criteria. In this study, our aim was to perform brain CT scanning at approximately 2 years after stroke. Of the 189 patients present at baseline, 136 were available for the last evaluation; 27 had died, 24 refused participation, 1 patient was untraceable, and 1 patient was too ill.

\section{CT-scan procedure}

The following features were recorded at baseline and at 2 years: infarct or hemorrhage, side of the symptomatic stroke (left or right hemisphere); lacunar or territorial infarct; presence of WMLs; presence of silent infarcts; brain atrophy. WMLs were defined as focal or diffuse hypodensities in the periventricular or deep white matter, not involving the cortex, with ill-defined margins to differentiate them from infarction [54]. The presence of WMLs around the frontal or occipital ventricular horns or in the centrum semi ovale was rated separately. Silent brain infarction was defined as a low-density area on CT, compatible with infarction but without a patient history of stroke, as determined from the patient, family, or other accessible information. Also, the stroke symptoms at study entry had to be anatomically incompatible with such silent infarcts or the lesions present on CT had to be old. Old infarcts can be distinguished from new infarcts by their greater hypodensity, and signs of surrounding tissue loss such as retraction of brain structures toward the infarct. The degree of atrophy was scored in a semi-quantitative way (none, mild, moderate, and severe), according to the procedure of Leys et al [55]. In short, the CT scans of four patients were selected during a consensus meeting by three experts as example scans for the four categories of no, mild, moderate, or severe brain atrophy. Then the CT scans of the study patients were graded by comparison with these 'control' scans. Two neurologists examined the CT scans independently. They were blinded for the neurological signs and the neuropsychological data. If there was a difference of opinion, consensus was reached by discussion. Inter-rater agreement (after omission of consensus reached by discussion) in the classifica- 
tion of patients by stroke features on baseline CT was good, with a $\mathrm{K}$ of 0.88 for symptomatic side of the infarct, $\mathrm{K}$ of 0.94 for hemorrhage, $\mathrm{K}$ of 0.85 for location, $\kappa$ of 0.60 for white matter, $\mathrm{K}$ of 0.79 for silent infarcts, and $\mathrm{\kappa}$ of 0.69 for atrophy. If there was no symptomatic stroke lesion visible on $\mathrm{CT}$, clinical data were used to categorize the stroke as lacunar or territorial. Fifty-one patients $(50.5 \%)$ had no symptomatic lesions on early CT. Clinical data were used to categorize these patients and those without symptomatic lesiosn on scan, as having lacunar or territorial brain infarction.

New infarcts, either silent or symptomatic, were rated if the CT at 2 years showed new lesions compatible with infarction. The neurologist who rated the second CT scan was blinded for the results of the first CT scan reading. Increase in WMLs was scored if patients showed white matter lesions not present at baseline, in at least one of the following three areas: around the frontal or posterior ventricle horns, or para-ventricular/centrum semi ovale areas. No effort was made to quantify the increase of WMLs in areas where WMLs were already present.

\section{Neuropsychological assessment}

The neuropsychological tests used have been described extensively in earlier reports $[29,101]$ 'Memory' was derived from the Auditory Verbal Learning Test (AVLT)[42]. 'Executive functioning' and 'mental speed' were based on a compound score of the Stroop Colour Word Test (SCWT) with the Concept Shifting Test (CST) [45, 82]. 'Calculation' and 'visuospatial abilities' were defined by subscales of the Groninger Intelligence Scale (GIT) [47]. 'Orientation', 'attention', 'praxis', 'language', and abstract reasoning, are subtests of the CAMCOG. The CAMCOG was used as a brief, objective assessment of cognitive functioning [39]. A decline of at least 5 points on the CAMCOG compared with the earlier score was defined as cognitive decline.

\section{Statistical Analyses}

Two groups of patients were formed: those in whom brain damage had progressed and those without such progression. We performed three analyses. First, analyses were performed to compare patient characteristics (demographic, clinical, and neuropsychological) between the two groups using chisquare analyses $\left(\chi^{2}\right)$ for dichotomous and T-tests analyses for continuous variables. We investigated whether patients with progressive brain damage showed cognitive decline after stroke more often than patients without such progressive brain damage. 
Secondly, we determined whether there were differences in cognitive functioning at assessment 1 , compared to assessment 2 , between patients with progressive brain damage and those without. Analyses of Variance (ANOVA) with repeated measures were conducted. For each cognitive domain an ANOVA was conducted with group (progressive vs non-progressive damage) as between subject factor, and time (baseline and follow-up cognitive performance) as a two-level within subjects factor.

Thirdly, the direction of change (improvement or deterioration) was tested posthoc using paired T-tests, for each group separately and for each cognitive domain (rough testscores) between measurement 1 and measurement 2. Significance was set at $\mathrm{p}<.05$ and all tests were performed two-tailed. Finally, in order to detect whether the results were influenced by outlining cases, the procedures described by Tatemichi et al. were followed. Analyses were performed with the Statistical Package for Social Sciences version 10 (SPSS-10).

\section{Results}

Of the 136 patients available at the last measurement, 101 participated. Of the non-participants, 13 patients had transport problems getting to the hospital, 15 patients refused participation, 5 patients were too ill, and 2 patients died before the CT could be made. Patients who did not undergo a second CT were older $(\mathrm{t}$ $=5.1, \mathrm{df}=1, \mathrm{p}=.00)$, had lower MMSE-scores on both measurements $(\mathrm{t}=-3.1$, $\mathrm{df}=1 . \mathrm{P}=.01 ; \mathrm{t}=-4.4, \mathrm{df}=1, \mathrm{p}=.00$ respectively), and more often had cortical infarcts at baseline $\left(\mathrm{X}^{2}=8.5, \mathrm{df}=1, \mathrm{p}=.01\right)$.

\section{Patient characteristics and cognitive functioning}

Of the 101 patients, 23 (22.7\%) had progressive brain damage compared to their baseline scan data. Of these patients two had had a recurrent stroke. Patient characteristics of the two groups are presented in table 1. 


\begin{tabular}{|c|c|c|c|}
\hline & $\begin{array}{l}\text { Data } \\
\text { Available } \\
\mathrm{N}\end{array}$ & $\begin{array}{l}\text { Progressive } \\
\text { damage } \\
(\mathrm{N}=23)\end{array}$ & $\begin{array}{l}\text { No progressive } \\
\text { damage } \\
(\mathrm{N}=78)\end{array}$ \\
\hline Age (mean, SD) & 101 & $70.2(10.1)$ & $60.6(10.8) *$ \\
\hline Level of education \% Low & 101 & $39.1 \%$ & $43.6 \%$ \\
\hline Sex \% Female & 101 & $65.2 \%$ & $39.7 \% *$ \\
\hline Infarct-Hemorrhage & 101 & $21 / 2$ & $70 / 8$ \\
\hline Left-Right & 96 & $7 / 15$ & $30 / 44$ \\
\hline Lacunar-Territorial & 90 & $17 / 4$ & $46 / 23$ \\
\hline Asymptomatic $\ddagger$ & 101 & $7 / 16$ & $20 / 58$ \\
\hline Leukoaraiosis $\ddagger$ & 101 & $4 / 19$ & $9 / 69$ \\
\hline Atrophy $\ddagger$ & 101 & $16 / 7$ & $39 / 39$ \\
\hline Hypertension $\ddagger$ & 101 & $12 / 11$ & $29 / 49$ \\
\hline Diabetes $\ddagger$ & 100 & $4 / 19$ & $9 / 68$ \\
\hline Hartfailure $\ddagger$ & 99 & $4 / 19$ & $8 / 68$ \\
\hline Cholesterol $\ddagger$ & 71 & $1 / 14$ & $11 / 45$ \\
\hline Current smoking $\ddagger$ & 101 & $5 / 18$ & $31 / 47$ \\
\hline Therapy $* * ~ \ddagger$ & 101 & $14 / 9$ & $40 / 38$ \\
\hline MMSE-score (mean, SD) & 101 & $25.8(2.8)$ & $26.6(3.1)$ \\
\hline
\end{tabular}


Table 2: presents 1-month and 2-year measurements of cognitive functioning, comparing patients with and without progressive brain damage.

Table 2: Differences cognitive test-performances between patients with and without progressive brain damage 1 month and 2 years post-stroke

\begin{tabular}{|c|c|c|}
\hline & $\begin{array}{l}\text { Progressive brain damage } \\
\text { Mean (SD) } \\
\mathrm{N}=23\end{array}$ & $\begin{array}{l}\text { No progressive brain damage } \\
\text { Mean (SD) } \\
\mathrm{N}=78\end{array}$ \\
\hline Memory $1 \bullet$ & $22.1(6.9)$ & $26.7(7.6) *$ \\
\hline Memory 2 • & $23.7(7.9)$ & $29.3(8.1)^{*}$ \\
\hline Executive $1 \ddagger$ & $61.1(34.4)$ & $47.2(30.7)$ \\
\hline Executive $2 \ddagger$ & $58.0(38.1)$ & $38.2(21.3) *$ \\
\hline Mental Speed $1 \ddagger$ & $46.4(12.0)$ & $46.1(18.1)$ \\
\hline Mental Speed $2 \ddagger$ & $46.1(10.5)$ & $39.7(12.3)$ * \\
\hline Calculation $1 †$ & $7.7(5.3)$ & $8.7(3.9)$ \\
\hline Calculation $2 \dagger$ & $8.6(5.3)$ & $9.8(3.5)$ \\
\hline Visuospatial $1 \dagger$ & $6.9(3.9)$ & $8.4(3.7)$ \\
\hline Visuospatial $2 \dagger$ & $7.5(3.6)$ & $8.9(4.3)$ \\
\hline Orientation $1 \dagger$ & $9.3(0.7)$ & $9.2(0.9)$ \\
\hline Orientation $2 \dagger$ & $9.3(0.8)$ & $9.5(1.0)$ \\
\hline Attention $1 \dagger$ & $7.5(1.6)$ & $7.8(1.7)$ \\
\hline Attention $2 \dagger$ & $7.7(2.0)$ & $7.8(1.7)$ \\
\hline Praxis $1 \dagger$ & $9.6(1.9)$ & $10.4(1.9)$ \\
\hline Praxis $2 \dagger$ & $10.3(1.6)$ & $11.0(1.3)$ * \\
\hline Language $1 \dagger$ & $25.2(2.7)$ & $25.5(3.0)$ \\
\hline Language $2 \dagger$ & $25.7(2.5)$ & $25.8(3.0)$ \\
\hline Abstract Reasoning $1 †$ & $12.4(2.4)$ & $13.6(2.3) *$ \\
\hline Abstract Reasoning $2 \dagger$ & $13.5(2.4)$ & $14.4(2.1)$ \\
\hline CAMCOG $1 \dagger$ & $85.5(8.0)$ & $88.2(9.6)$ \\
\hline CAMCOG $2 \dagger$ & $88.4(8.4)$ & $91.4(9.9)$ \\
\hline MMSE $1 †$ & $25.9(2.8)$ & $26.6(3.1)$ \\
\hline MMSE $2 \dagger$ & $26.0(3.1)$ & $26.8(3.1)$ \\
\hline \multicolumn{3}{|c|}{$\begin{array}{l}1=1 \text { month post-stroke } \mid 2=2 \text { years post-stroke }\left|{ }^{*} p<.05\right| \cdot \text { Number of words } \\
\text { reported } \mid \neq \text { Seconds needed to complete the task } \mid \dagger \text { Number of correct responses }\end{array}$} \\
\hline
\end{tabular}


On average, patients with progressive brain damage performed worse than those without such damage. While this was true for all cognitive domains, the difference was only statistically significant for memory performance at 1 month and 2 years after stroke, for abstract reasoning 1 month after stroke, and for executive functioning, mental speed, and praxis at 2 years follow-up.

\section{Changes in cognitive functioning}

Table 3 shows the relation between progressive brain damage and cognitively decline or improvement.

Table 3: Number and Percentages of patients declining, improving or stable, measured with CAMCOG

\begin{tabular}{lcc}
\hline & \multicolumn{1}{c}{ Progressive brain damage } & \multicolumn{1}{l}{ No Progressive brain damage * } \\
& $\mathrm{N}(\%)$ & $\mathrm{N}(\%)$ \\
\hline Decline & $2(8.7)$ & $6(7.7)$ \\
Improve & $8(34.8)$ & $30(38.5)$ \\
Stable & $13(56.5)$ & $42(53.8)$ \\
\hline${ }^{*} \chi^{2}=.11 \mathrm{df}=2, \mathrm{p}=.89$ & \\
\hline
\end{tabular}

Progressive brain damage did not influence cognitive function measured with the CAMCOG.

Cognitive functioning at 1 month after stroke compared to 2 years later is presented in table 4 . The left part of table 4 presents data about whether cognitive changes in patients with progressive brain damage differ from patients without progressive brain damage. The second part of the table presents the direction of change in each group separately. 
Table 4: Cognitive functioning 1 month compared to 2 years and direction of change

\begin{tabular}{|c|c|c|c|c|c|c|c|}
\hline & \multirow{2}{*}{\multicolumn{3}{|c|}{ Course $^{1)}$}} & \multicolumn{4}{|c|}{ Direction of change 2) } \\
\hline & & & & Progressive & damage & \multirow{2}{*}{\multicolumn{2}{|c|}{$\begin{array}{l}\text { No progressive damage } \\
\text { Mean (SD) }\end{array}$}} \\
\hline & \multirow[t]{2}{*}{$\mathrm{F}$} & \multirow[t]{2}{*}{$\mathrm{df}$} & \multirow[t]{2}{*}{$\mathrm{p}$} & \multicolumn{2}{|c|}{ Mean (SD) } & & \\
\hline & & & & Baseline & 2 years & Baseline & 2 years \\
\hline Memory & .66 & 1,92 & .42 & $22.1(6.9)$ & $24.1(7.9)$ & $26.5(7.6)$ & $29.5(7.9)^{*}$ \\
\hline Executive & .09 & 1,88 & .77 & $60.9(35.4)$ & $50.2(27.7)^{*}$ & $46.8(31.2)$ & $37.8(21.4)^{*}$ \\
\hline Mental Speed & 3.3 & 1,91 & .08 & $46.4(12.0)$ & $45.0(10.3)$ & $45.8(18.3)$ & $39.1(11.9)^{*}$ \\
\hline Calculation & .52 & 1,81 & .47 & $8.2(5.6)$ & $8.8(5.5)$ & $8.9(3.8)$ & $10.0(3.5)^{*}$ \\
\hline Visuospatial & .01 & 1,87 & .92 & $7.0(4.1)$ & $7.5(3.9)$ & $8.5(3.7)$ & $8.9(4.3)$ \\
\hline Orientation & 1.3 & 1,99 & .26 & $9.3(0.7)$ & $9.3(.8)$ & $9.2(0.9)$ & $9.5(0.9)$ \\
\hline Attention & .46 & 1,99 & .49 & $7.5(1.6)$ & $7.7(2.0)$ & $7.8(1.7)$ & $7.8(1.7)$ \\
\hline Praxis & .01 & 1,99 & .93 & $9.6(1.9)$ & $10.3(1.6)$ & $10.4(1.9)$ & $11.0(1.3)^{*}$ \\
\hline Language & .06 & 1,99 & .80 & $25.2(2.7)$ & $25.7(2.5)$ & $25.5(3.0)$ & $25.8(3.0)$ \\
\hline Abstract & .42 & 1,97 & .52 & $12.4(2.4)$ & $13.5(2.4)^{*}$ & $13.6(2.4)$ & $14.4(2.1)^{*}$ \\
\hline MMSE & .01 & 1,97 & .95 & $25.9(2.8)$ & $26.0(3.1)$ & $26.6(3.1)$ & $26.8(3.1)$ \\
\hline CAMCOG & .03 & 1,99 & .87 & $85.5(8.0)$ & $13.5(2.4)^{*}$ & $88.2(9.6)$ & $91.4(9.9)^{*}$ \\
\hline
\end{tabular}

${ }^{*} \mathrm{p}<.05 \mid$ 1) ANOVA | 2) POST-HOC Students T tests

Basically, the changes in cognitive functioning at 1 month and 2 years were similar in the two groups, with both groups showing improvement. This improvement was only significant for executive functioning and abstract reasoning in both groups, and for memory, mental speed, calculation, praxis, and overall cognitive functioning measured with the CAMCOG for the patients without progressive brain damage. After correction for baseline characteristics possibly related with cognitive performance (age, educational level, type and side of the infarct and stroke risk factors), post hoc analyses yielded similar results.

\section{Discussion}

The present study showed that, despite standard secondary prevention treatment, brain damage had progressed in more than $20 \%$ of the stroke patients after 2 years, consistent with earlier findings [54]. Although patients with progressive brain damage performed worse than those without such brain damage, in most patients cognitive performance remained stable or had even improved 2 years after stroke.

Risk factors such as hypertension, genetic factors, and baseline extent of WMLs 
have been associated with progression of WMLs over time [158]. Although more patients with progressive brain damage had hypertension, the difference was not statistically significant, probably due to the relatively small sample.

Thus we found no evidence to support our hypothesis that progressive brain damage is related to a decline in cognitive performance following first-ever stroke. Meyer et al. found that patients with $\mathrm{VaD}$ whose cognitive performance decreased over time, more often had recurrent infarcts and changes in cerebral perfusion than those whose cognitive performance remained stable [156]. In general, patients with $\mathrm{VaD}$ show a decline in cognitive functioning [91, 156, $159,160]$ whereas stroke patients with mild cognitive deficits show a substantial improvement in cognitive functioning with time [89, 91, 113, 123, 125, 159]. In our study, the cognitive functioning declined in about $8 \%$ of the patients but improved in more than $33 \%$. Apparently, the relation between progressive brain damage and cognitive functioning in a stroke population without dementia is not as straight forward as it is in patients with $\mathrm{VaD}$. However, patients with progressive brain damage improved on fewer cognitive domains $\left(\mathrm{N}_{\text {improvement }}=\right.$ 3) than those without progression $\left(N_{\text {improvement }}=7\right)$ but the course of cognitive functioning was similar in both groups. So even if we did not found evidence for our hypothesis, patients with progressive brain damage performed worse and showed on fewer cognitive domains improvement, compared to patients without progressive brain damage. The explanation of the lack of support for our hypothesis could be that the 2-year follow-up period was too short to detect a deterioration of cognitive functioning. Moreover, a decline in cognitive functioning may become manifest only when the amount of ischemic brain damage has reached a certain threshold. Besides this, the patients included in the study did not have very severe impairments in cognitive functioning because we excluded patients with an MMSE score $<15$, severe aphasia, or pre-stroke dementia at baseline and included both patients with VCI and those without cognitive deficits. To find out whether the inclusion of both patients with and without cognitive deficits influenced our results, we repeated the analysis restricted to data for patients with VCI. Again cognitive performance improved in all patients regardless of the presence of progressive brain damage. Thus the inclusion of patients without cognitive deficits did not substantially influenced our results. The present study had some shortcomings. Firstly, we used CT to detect brain damage instead of the more sensitive technique of magnetic resonance imaging (MRI). However, CT may be more specific than MRI, and as such the chance of false positive findings is likely to be low. A second point is that patients were tested four times, which could have led to a learning effect. Although this effect could not be ruled out, it is minimal for the tests we used [93]. Thirdly, 35 patients did not undergo the repeated CT at 2 years. These patients may have had a higher chance of having progressive brain damage because they had lower cognitive performance at baseline. If we could have included these patients, the 
difference between patients with progressive brain damage and those without might have been more pronounced.

In conclusion, although cognitive functioning was worse in patients with progressive post-stroke brain damage than in patients without such damage, functioning over time was not related to progression of ischemic brain damage. Thus, for stroke survivors with minor or without cognitive impairment, there appears not to be a simple relation between an increase in 'silent' ischemic brain damage and cognitive functioning. 


\section{XI}

\section{Checklist for cognitive and emotional}

consequences following stroke (CLCE-24): development and initial validation

\section{Adapted from}

Rasquin SMC, Heugten van CM, Winkens I, Beusmans G, Verhey FRJ. CheckLijst voor het opsporen van cognitieve en emotionele gevolgen na een beroerte (CLCE-24). Nederlands Tijdschrift voor Geneeskunde. Herziene versie ingediend. 


\section{Abstract}

Background: Cognitive and emotional problems are common after stroke and screening is essential. In this paper a new screeninginstrument is presented and aspects of the feasibility and validity are investigated.

Methods: A group of stroke patients are interviewed with the new instrument, CLCE-24, six months post stroke. Validity was determined using reference instruments (MMSE, CAMCOG, SCL-90).

Results: Sixtynine patients participated in the study (37 men; mean age 66). The interview with the CLCE-24 took 11 minutes. Patients and assessors are positive about its use. Eighty percent had cognitive and/or emotional problems; 73\% had cognitive problems, while $51 \%$ had emotional problems. The number of complaints varied between 0 and 14 . Attention, mental slowness and memory were the most reported cognitive complaints; depression and fear were the most reported emotional complaints. Correlations between the CLCE-24 and MMSE, CAMCOG and SCL-90 were significant. The CLCE-24 is a significant predictor of the MMSE (Adj $R^{2}=0.31$ ) and CAMCOG (Adj $R^{2}=0.16$ ) at 12 months post stroke. The sensitivity is above $80 \%$, the specificity varies between 22 and $47 \%$. Conclusions: The CLCE-24 is a feasible and valid instrument to be used for cognitive screening by non-psychologists. Further research and implementation in clinical practice is recommended. 


\section{Introduction}

The last two decennia it has become increasingly clear that the non-physical (i.e. cognitive and emotional) consequences following stroke are not some rare or exotic phenomenon affecting only a few survivors. Quite the reverse is true: the study of Tatemichi [32] revealed that $78 \%$ of 227 stroke patients were left with cognitive problems. Others found that more than $50 \%$ of the patients suffered from mental slowness, whereas 20 to $40 \%$ of the stroke patients had memory, visuospatial and constructional, language, and arithmetic problems [29,161]. Aben et al. [162] studied post stroke depression and found a cumulative one year incidence of major and minor depression of $37.8 \%$ in a group of 190 first ever stroke patients.

Cognitive and emotional dysfunction will result in considerable functional problems and may hinder the outcome of rehabilitation even up to four to six years after stroke $[32,163,164]$. Because of this huge negative impact on daily life, it is essential to assess stroke patients for these less visible problems in all phases after stroke (i.e. acute, sub-acute and chronic). Extensive neuropsychological testing however, is time-consuming, can be exhausting for the patient and is not relevant in the acute phase after stroke in cases being medically unstable or changing dramatically during the first weeks $[165,166]$. In the chronic phase, when patients return home, there is often no neuropsychologist or neurologist involved, and as such psychological problems remain unrecognized. Cognitive screening (1 to 2 hours testing time) instead of extensive testing should therefore be considered. In the chronic phase after stroke however, there is often no neuropsychologist or neurologist involved. Identifying psychological problems in this phase needs to be conducted by other healthcare professionals, such as the general practitioner, community nurse or physiotherapist. Suitable screeninginstruments are not available: existing instruments were originally designed for other purposes, mostly screening for the presence of dementia. Moreover, general practitioners, community nurses or physiotherapists often have a lack of knowledge about how to assess these psychological consequen-ces. The purpose of the present study therefore was to develop a checklist for identification of cognitive and emotional consequences in the chronic phase after stroke, which can be used by professionals other than the trained psychologist or neurologist. In this paper the development and initial validation of a new instrument are described. 


\section{Methods}

\section{Patients}

Patients recruited for the CODAS study (Cognitive Disorders after Stroke) were asked to participate in the present study. The CODAS project is a prospective, longitudinal, observational study in which 198 consecutive stroke patients with a first cerebral stroke admitted to the University Hospital Maastricht, are followed neuropsychologically at 1, 6, 12 and 24 months after stroke, investigating the course of cognitive functioning and risk factors for cognitive impairments [29].

Patients were asked to participate in the CODAS study within 48 hours after their stroke and were included on the basis of the following criteria: a first cerebral stroke, age older than 40 years (to avoid atypical strokes) and an MMSE score higher than 14 (to avoid severe cognitive deterioration and/or severe aphasia). Stroke was diagnosed clinically by a neurologist, supported by a CTscan. Exclusion criteria were other major neurological or psychiatric disorders which could lead to cognitive impairments. For the present study, one extra inclusion criterion was that patients were living at home six months after their stroke.

All patients received verbal and written information about the study and gave written consent to participate. The research protocol was approved by the local medical ethical committee.

\section{Assessments}

The Checklist for cognitive and emotional consequences following stroke, the CLCE-24, was developed on the basis of existing instruments, literature searches and consultation of experts, using the following criteria: multidimensionality assessing cognitive domains as well as emotional consequences following stroke, suitable to be used by non-psychologists, focussing on the patients' problems since the occurrence of the stroke, supporting a qualitative judgment instead of generating a score representing presence or absence of problems, and short and easy to use. Twenty-two items are defined representing cognitive and emotional complaints (for instance 'since the stroke the patient has problems doing two things simultaneously'). Each item is supported by questions to ask the patient and behaviours to observe or notice (e.g. not being able to cook and talk, or walk and talk at the same time; the patient does not answer any questions while taking of his coat). The last two items are not specified in order to 
allow other problems to be discussed by the patient (total 24 items). For each item the presence or absence can be indicated and the severity is scored (e.g. hindering daily life or not). The checklist is filled in by interviewing the patient and/or an informal caregiver. For each item the interviewer can indicate who reports the problem (patient, caregiver, interviewer). The professional is recommended to use the CLCE-24 for all stroke patients on a routine basis, and not to wait for problems to develop. In addition, if a problem is present, the interviewer asks about the influence it has on daily life (e.g. severely hindering daily life). All questions were asked in a standardised manner, relying on the extra information given for each item. A concept version of the CLCE-24 was used in this study; the final version, after revision, is presented in Appendix 1.

Reference instruments used in the CODAS study: The Mini Mental State Examination (MMSE) [41] and the Dutch CAMCOG, part of the Cambridge Examination for Mental Disorders in the Elderly (CAMDEX) which assess cognitive functioning [39, 40, 50, 65]. The Symptom checklist-90 (SCL-90) [167] is used for psychopathological complaints.

\section{Procedure}

In the CODAS study patients were contacted by telephone six months after stroke to take part in the second assessment. Patients were informed about the purpose and duration of the CLCE-24 and asked to participate in this study as well. The interview according to the CLCE- 24 always preceded the neuropsychological testing during a visit by a psychologist. Afterwards, patients, relatives, and assessors were asked about their experience with the new instrument.

\section{Statistical analyses}

The total CLCE-24 is considered, as well as the cognitive and emotional items separately, depending upon the reference instrument. Descriptive statistics, to describe patient characteristics, correlation coefficients, to define the raltion between the CLCE-24 and the MMSE, CAMCOG, or SCL-90 and t-tests, to compare patients with at least 1compalint to patients without complaints were used. The sensitivity and specificity of the CLCE-24 are determined on the basis of the MMSE and CAMCOG. The cut-off points for the MMSE are based on the presence of cognitive impairments, which could indicate dementia (MMSE < 24) [168] and the presence of cognitive impairments in patients referred to an outpatient memory clinic (MMSE < 27) [126, 168]. The cut-off points for the 
CAMCOG are based on cut-off points for cognitive problems in relation to the presence of dementia $(C A M C O G<80)[50]$ and the mean of the present population (CAMCOG < 88.9). Comparison between the CLCE-24 and the SCL-90 was not taken into account because the number of items covering the emotional domain of the CLCE-24 ( $=7)$ was not balanced to the 90 items of the SCL90. In defining sensitivity and specificity, no cut-off score of the CLCE-24 was used, as the purpose of this instrument is to give a qualitative judgement about the presence of psychological problems and not to give a cut-off for diagnostic purposes. Instead, presence of at least 1 psychological problem (either cognitive or emotional) was compared to absence of any problems. Investigation of the predictive accuracy of the CLCE-24 was done using linear regression analyses.

\section{Results}

\section{Patients}

In total 69 patients were included in the period between April 2001 and January 2002. The characteristics of the patients are shown in table 1.

\begin{tabular}{ll}
\hline Table 1: Patients characteristics & \\
\hline Characteristics & $\mathrm{N}$ \\
Sex & $37 / 32$ \\
$\mathrm{M} / \mathrm{F}$ & $66.1(12.3)$ \\
Age (mean/sd) & \\
$\begin{array}{l}\text { Education } \\
\text { low/middle/high } \\
\text { Type of stroke }\end{array}$ & $35 / 24 / 10$ \\
$\begin{array}{l}\text { Haemorrhage/cortical/lacunar infarction } \\
\text { Test location }\end{array}$ & $5 / 43 / 21$ \\
$\begin{array}{l}\text { Home/hospital } \\
\text { Test situation } \\
\text { Patient/ patient with family member } \\
\text { Living situation } \\
\text { With partner/alone/otherwise }\end{array}$ & $51 / 18$ \\
\hline SD $=$ Standard Deviation & $48 / 21$ \\
\hline
\end{tabular}




\section{Feasibility of the CLCE-24}

On average it took 11.1 minutes (sd 4.5; range 5-35 minutes) to interview the patient using the CLCE-24. Seventy-eight percent of the interviews was done in less than 10 minutes. The assessors asked the patients their opinion about the interview with the CLCE-24 in a structured way. There were many positive reactions (list is clear, it concerns relevant items, it is good to ask these questions, the items are recognizable and realistic); some patients commented that it should be standard procedure to ask about these problems. None of the patients reported that the list was either too long, or too personal. The assessors in this study said the CLCE-24 was quick to understand, simple to use, useful, relevant, and sometimes the interview led to very emotional conversations showing many problems.

\section{Problems scored with the CLCE-24}

Considering presence of problems (i.e. no severity) eighty percent of the patients had one or more cognitive or emotional problems as assessed by the CLCE-24; $51 \%$ reported having 2 or more problems. Seventy-three percent had cognitive complaints, while $51 \%$ reported emotional problems. The number of complaints varied between 0 and 14. The mean number of problems was 3.3 (median 2.0); the mean number of cognitive and emotional problems were 2.3 (median 2.0) and 1.0 (median 1.0) respectively. The number of patients having problems per item is shown in table 2. Attention, mental slowness and memory were the most reported cognitive complaints. Depression and fear were the most reported emotional complaints. 
Table 2: Cognitive and emotional problems

Problems since stroke

$\mathrm{N}(\%)$

\section{Cognitive problems}

1. Awareness

2. Understand language

3. Speaking or writing

4. Social aspects of language

5. Perceiving time

$10(14.5)$

6. Orientating to places or persons

7. Keeping up; has become slower

8. Attending to things

$19(27.5)$

9. Doing two things at once

$21(30.4)$

10. Remembering new information

11. Remembering old information

12. Taking initiative

13. Planning and organizing things

14. Performing daily activities

15. Attending to a part of the body or space

\section{Emotional problems}
16. Decorum
$0(0.0)$
17. Less oriented socially
$11(15.9)$
18. Depressed
19. Manic depression
20. Not realistic about things
$11(15.9)$
21. In fear of things to come
$20(29.0)$
22. Less in control of his own behaviour
$4(5.8)$

The items in this table are based on the concept instrument which was tested and therefore do not completely match the final instrument in appendix 1.

The last two items on the CLCE- 24 concerned problems the patient might have, which were not yet mentioned in the interview: problems when many people are talking together (2), angry easily (2), getting less emotional (2), being more emotional (4).

There appear to be no significant differences between patients with $(n=55)$ and without problems $(n=14)$ concerning age or education. Women report to have 
more problems (mean number of complaints for men 2.6 and for women 4.2; $\mathrm{p}=0.04$ ). There are no significant differences concerning age, gender and education in terms of cognitive problems, but women appear to have more emotional problems (mean number of complaints for men 0.6 and for women 1.5; $\mathrm{p}<0.01$ ).

\section{Validation of the CLCE-24}

The correlations between the scores on the CLCE-24 and the CAMCOG and MMSE at six months post stroke were significant: $r=-0.31(p<0.01)$ and $r=-0.27$ $(\mathrm{p}<0.05)$ respectively, thus indicating problems in functioning on all measures. Comparing the cognitive items of the CLCE-24 to the CAMCOG and MMSE shows weaker relationships: $\mathrm{r}=-0.20(\mathrm{p}=0.10)$ and $\mathrm{r}=-0.21(\mathrm{p}=0.08)$ respectively. The emotional items of the CLCE-24 correlated higher with MMSE ( $\mathrm{r}=-0.30, \mathrm{p}<$ $0.05)$ and CAMCOG $(r=-0.43, p<0.05)$. The CLCE- 24 also correlates significantly with the SCL-90 $(r=0.57 ; p<0.01)$; the same is true for comparison of the emotional items of the CLCE-24 to the SCL-90 $(r=0.51 ; \mathrm{p}<0.01)$. The cognitive items of the CLCE-24 correlate high with the SCL-90 $(r=0.52, \mathrm{p}<0.05)$.

Patients having problems on the CLCE-24, have a lower but not significant score on the MMSE ( $t=1.80 ; p=0.08$ ) in comparison to patients reporting no problems at all. Patients having problems on the CLCE-24 have a significant lower score on the CAMCOG $(\mathrm{t}=3.08 ; \mathrm{p}=0.00)$. Patients reporting cognitive problems on the CLCE-24 have a significant lower score on the MMSE $(t=2.52 ; p=0.01)$ and on the CAMCOG $(\mathrm{t}=2.45 ; \mathrm{p}=0.02)$ compared to patients having no cognitive complaints.

\section{Predictive properties of the CLCE-24}

In order to determine whether the CLCE-24 can predict scores on the MMSE and CAMCOG six months later, regression analyses were performed, controlling for gender as women presented more problems on the CLCE-24 than men. The results show that the CLCE-24 is a significant predictor of the MMSE (Adj $\left.\mathrm{R}^{2}=0.31 ; \mathrm{p}<0.01\right)$ and the CAMCOG (Adj. $\left.\mathrm{R}^{2}=0.22 ; \mathrm{p}<0.01\right)$ at 12 months after stroke. The same is true for the cognitive part of the CLCE-24 and the MMSE score (Adj $R^{2}=0.28 ; p<0.01$ ) and the CAMCOG (Adj. $R^{2}=0.16 ; p<0.01$ ). These results indicate that patients reporting problems on the CLCE-24 at six months after stroke also have more problems at twelve months after stroke as measured with the MMSE and CAMCOG. 


\section{Sensitivity and specificity of the CLCE-24}

Using 24 as the traditional cut-off point for the MMSE, the sensitivity is $88.9 \%$ and the specificity is $21.7 \%$; comparing the MMSE to the presence of cognitive problems on the CLCE-24, the sensitivity is $88.8 \%$ and the specificity is $30 \%$. Using 27 as cut-off point, the sensitivity is $92.9 \%$ and the specificity is $29.3 \%$; for cognitive problems, the sensitivity is $85.7 \%$ and the specificity is $36.6 \%$.

Based on a cut-off point of 80 for the CAMCOG, the sensitivity of the CLCE-24 is $90.9 \%$, the specificity is $22.4 \%$. Using the mean as cut-off point (89) the sensitivity is $85.7 \%$ and the specificity is $47.3 \%$. Considering only the cognitive problems on the CLCE-24, the sensitivity and specificity for a cut-off point of 80 are $81.8 \%$ and $29.3 \%$ respectively. For the cut-off point 89 , the sensitivity and specificity are $73.9 \%$ and $46.0 \%$ respectively.

\section{Discussion}

Examining a group of 69 patients, the feasibility and aspects of the validity of a new checklist (CLCE-24) for psychological problems after stroke were determined. The CLCE-24 is suitable for clinical practice, because the instrument is easy to use and can be used to assess the psychological problems from a patients' perspective adequately.

The CLCE-24 was developed on the basis of a set of criteria, of which the following were met: short and simple, focussing on the problems of the patient, and assessing cognitive and emotional consequences. Patients and assessors were positive about the instrument and the time needed for the assessment is limited. The use of the concept instrument has led to some minor changes. Four items were removed because these appeared to be less relevant in this group and were not found in stroke patients in other studies either (i.e. manic depression) or were not suitable to ask the patients themselves (i.e. awareness, social aspects of language and decorum). Four new items were added because these were mentioned by patients and found to be relevant from other studies on frequency of complaints (emotionally less stable, irritated, less emotional and tired quickly) [161]. In addition, the order of items was changed according to incidence in the present group as well as other groups studied [29, 101,161]. Finally, concerning the scoring of the items, an indication of the severity of a problem (i.e. the impact on daily life) was added in order to be able to differentiate better in terms of care needed. Although the final version of the CLCE-24 is somewhat different from the version we studied, to our opinion this has no effect on the conclusions as the changes were minor.

Patients presenting complaints during the interview with the CLCE-24, also have lower scores on the reference instruments. Patients expressing cognitive 
and emotional problems, also perform worse on the MMSE and CAMCOG six months later. These results imply that the CLCE-24 can be used adequately to identify subjective for the neuropsychological consequences as experienced by the stroke patient and can predict possible problems at a later stage after the stroke. However, it should be mentioned that the correlations between the CLCE-24 and MMSE/CAMCOG were low, but significant. Moreover, the correlations between the cognitive part of the CLCE-24 were higher with the SCL-90 compared to the MMSE or CAMCOG. This could be explained by the fact that instruments like the MMSE and CAMCOG are not suitable to identify patients with minor cognitive deficits.

Concerning the diagnostic value of the CLCE-24, we found sufficient sensitivity but poor specificity values. A combination of high values on both these diagnostic aspects is preferable, but since the instrument is aimed at identifying possible problems, the focus is on the sensitivity. Since the CLCE-24 is developed for identification of potential problem areas after which neuropsychological testing will be used to further assess the impact of the actual problems, this is not a problem. Probably the most realistic cut-off point to compare the CLCE-24 with is 27 on the MMSE, as these are the patients with mild cognitive deficits [126, 165]. This cut-off point was found to be predictive in patients developing Alzheimers disease over 3 to 5 years, starting with a preclinical stage affecting cognitive functioning mildly. Applying this cut-off point, the percentage of patients classified as having cognitive problems on the basis of the CLCE-24 then is $93 \%$ for the total scale and $86 \%$ for the cognitive aspects.

The professional is recommended to use the CLCE-24 for all stroke patients on a routine basis, and not wait for problems to develop. The CLCE-24 has some advantages compared to the existing cognitive screenings tests. These tests are mostly developed to identify patients at risk for developing dementia, and applying them to patients with minor deficits would make no sense as minor deficits are not recognized. In case a patient reports a problem on the CLCE-24, either cognitive or emotional, this problem has to be elaborated by the professional, and referral to a specialist (just as a neuropsychologist or psychiatrist) should be considered.

The results of the CLCE- 24 presented here are promising and future research is justified. Evidently, the use of the instrument by non-psychologists has to be investigated further.

In addition, testing the instrument in a larger multi-centre study focussing on the test-retest reliability is a preferable next step. Furthermore, as the CLCE-24 was developed to only identify psychological problems and not for diagnostic purposes, criteria for referral to psychologists for further interventions are still to be formulated. For this reason, in the final version of the CLCE- 24 the severity of the reported problems is incorporated and could be used as an indication for referral: if a patient and/or the family report that a problem seriously 
hinders daily life, further steps are necessary. Psychologists can decide to do an extensive neuropsychological assessment, offer psycho-education, or even start specific cognitive training programs. Several studies have shown that stroke patients and families have many questions concerning the consequences of the stroke, especially in the area of cognition, emotion and behaviour [169, 170]; information provision can be expected to reduce distress and burden of caregivers. Until now, no suitable screening instrument for adequate referrals was available.

In addition, in the present study the CLCE-24 was used to interview the stroke patients themselves, while partners were present in some cases. It is however important to interview the stroke patients and their caregivers separately since it is known that the cognitive and emotional complaints which are put forward can differ dramatically between the spouses: in studies by [161] and [171] it was found that the patients and their partners did not agree on most consequences of the stroke.; in both studies the partners report even more problems than the patients themselves. Family life in which problems are perceived differently can lead to conflicting situations and adequate counselling is needed. A new research study should therefore interview patients and caregivers independently.

Finally, because of the high incidence of psychological consequences following stroke, implementing the CLCE-24 (after some additional research) systematically in a stroke service could help reduce the number of patients and caregivers developing serious problems and needing additional care. From a study on dementia, it appeared that community nurses and general practitioners do not identify large groups of demented patients correctly and even rated depressed patients mistakenly as demented [172]. Early diagnosis and practical assistance can help to alert the responsible authorities before the patients and their families become unable to cope with the situation [172]. A comparison can be made with cognitive consequences following stroke: early (before situations might have gotten out of hand for the family) and standard (for every patient) screening can help to prevent the impact of the psychological aspects in daily life. 


\begin{tabular}{|l|l|l|l|l|}
\hline Appendix 1: Items of the CLCE-24, final version & $\begin{array}{l}\text { Yes, } \\
\text { Severely } \\
\text { hinder- } \\
\text { ing daily } \\
\text { life * }\end{array}$ & $\begin{array}{l}\text { Yes, } \\
\text { but not } \\
\text { severe * }\end{array}$ & Not & $\begin{array}{l}\text { I am * } \\
\text { sure }\end{array}$ \\
\hline Items & & & & \\
\hline $\begin{array}{l}\text { Since the stroke the patient has prob- } \\
\text { lems: } \\
\text { (cognitive problems) }\end{array}$ & & & & \\
\hline 1. doing two things at once & & & & \\
\hline 2. attending to things & & & & \\
\hline 3. keeping up; has become slower & & & & \\
\hline 4. remembering new information & & & & \\
\hline 5. remembering old information & & & & \\
\hline 6. taking initiative & & & & \\
\hline 7. planning and organising things & & & & \\
\hline 8. performing daily activities & & & & \\
\hline 9. perceiving time & & & & \\
\hline 10. orienting to places or persons & & & & \\
\hline 11. understanding language & & & & \\
\hline 12. speaking or writing & & & & \\
\hline $\begin{array}{l}\text { 13. attending to a part of the body or } \\
\text { space }\end{array}$ & & & & \\
\hline $\begin{array}{l}\text { Since the stroke the patient is: } \\
\text { (emotional problems) }\end{array}$ & & & & \\
\hline 14. depressed & & & & \\
\hline 15. in fear of things to come & & & \\
\hline 16. less oriented socially & & & \\
\hline 17. not realistic about things & & & \\
\hline 18. emotionally less stable, cries easily & & & \\
\hline 19. irritated, angry more easily & & & \\
\hline 20. less emotional, uninterested & & & \\
\hline 21. less in control of his own behaviour & & & \\
\hline 22. tired quickly & & & \\
\hline Are there any other problems? & & & \\
\hline 23. & & & \\
\hline 24. & & & \\
\hline * Scoring: patient, c caregiver, I interviewer & & \\
\hline
\end{tabular}




\section{XII}

\section{The influence of psychiatric symptoms on cognitive performance after stroke}

Adapted from

Rasquin SMC, Lodder J, Verhey FRJ. The influence of psychiatric symptoms on cognitive performance after stroke. Cerebrovascular Diseases. Revised version submitted. 


\section{Abstract}

Background: Stroke-patients often have neuropsychiatric symptoms and cognitive deficits. The aim of this study was to investigate whether stroke patients with psychiatric symptoms are at increased risk of developing cognitive deficits.

Methods: Cognitive function (assessed with a neuro-psychological test battery) and presence of psychiatric symptoms (assessed with the SCL-90) were evaluated at 1, 6, 12, and 24 months after stroke.

Results: At baseline, 156 patients entered the study, 15 were diagnosed with $\mathrm{VaD}, 113$ with post-stroke MCI. Patients with psychiatric symptoms were found to be at increased risk of being diagnosed with vascular dementia at baseline $(\mathrm{OR}=6.9 \mathrm{CI}=1.3-36.8)$ and decline more on cognitive function 6 month after stroke.

Conclusions: patients with psychiatric symptoms after stroke are at increased risk of cognitive deficits. 


\section{Introduction}

Stroke can have dramatic consequences for the patient and his or her environment, many patients experience not only motor but also cognitive impairments after a stroke $[2,6,32,99,101]$. The prevalence of Vascular Dementia $(\mathrm{VaD})$ varies from 8 to $22 \%$ and is increased by 9 -fold after stroke [2-6]. Besides cognitive impairment, stroke also increases the risk of neuropsychia-tric disorders [173-175]. For instance, depression is common after stroke, with prevalence up to $64 \%[162,176]$.

Although there is considerable information available on the development of both cognitive and psychiatric disorders after stroke, little is known about the interaction between the two. Only a few studies have investigated the association between neuropsychiatric deficits and cognitive performance after stroke. These studies found that the presence of depression predicted poor cognitive performance [129, 177-180]. However, most of these studies defined cognitive performance in terms of the Mini Mental State Examination (MMSE) score, which is only a rough indicator of cognitive functioning. Moreover, the MMSE is often biased toward language and memory domains [181], whereas other cognitive domains, such as mental speed, are not represented. Up until now the association between a broad range of neuropsychiatric symptoms and cognitive functioning has not been investigated.

The aim of this study was to investigate the relation between psychiatric symptoms and cognitive performance following stroke. We investigated whether the presence of psychiatric symptoms predicts the development of cognitive deficits. As cognitive deficits are common in patients with psychiatric symptoms [182-184], we hypothesized that psychiatric symptoms are associated with an increased risk of subsequent cognitive decline.

\section{Methods}

\section{Procedure}

The present study was a 2-year follow-up study, with 1, 6, 12, and 24 months assessment of a cohort of patients who suffered from first-ever supratentorial ischemic stroke. Baseline measures include, besides demographic and neurological variables, an assessment of psychiatric functioning (SCL-90) and cognitive testbattery. Both the SCL-90 and the testbattery were administered at each assessment. Primary outcome was cognitive status. 


\section{Patients}

Patients were participants of the CODAS-study (COgnitive Disorders After Stroke), a prospective study, which has been described earlier [29, 100, 101]. Patients were included in the study if they had had a first-ever hemispheric stroke, were older than 40 years, had post-stroke adequate fluency in Dutch, and had an initial MMSE $\geq 15$ (to ensure neuropsychological testing is possible). Severe aphasia, neurological deficits other than stroke, or major psychiatric disorders were exclusion criteria. The Medical Ethics Board of the University Hospital Maastricht approved the study, and all participants gave informed consent.

Patients were asked within 48 hours of stroke to participate in the study and were administered a clinical and neuropsychological examination at 1, 6, 12, and 24 months post-stroke.

\section{Medical Assessment}

Medical information was collected directly after patients came to the hospital because of stroke symptoms, and entered into the Maastricht Stroke Register (MSR), a prospective databank. This databank contains information on all stroke patients referred to the Department of Neurology of the University Hospital of Maastricht. This database includes data about neurological examination, brain imaging, clinical information, risk factors such as the presence of diabetes, hypertension, cholesterol, heart failure in the past, family history of vascular diseases and clinical diagnosis of the stroke-syndrome and the CT/MRI-scan data.

\section{Cognitive Domains}

The neuropsychological tests used in this study have been described extensively earlier $[29,49,101]$. The selection of the domains that were studied, was based on the criteria for $\mathrm{VaD}$, proposed by the National Institute for Neurological Disorders and Strokes - Association Internationale pour la Recherche et l'Enseignement en Neurosciences (NINDS-AIREN) [24]. 'Memory' was derived from the Auditory Verbal Learning Test (AVLT) [42], in which the total number of words recalled is the variable of interest. 'Executive functioning' was based on a combination of the interference score of the Stroop Colour Word Test (SCWT) [45] with the interference score of either the Concept Shifting Test (CST) [82]. 'Calculation' (doing as many sums as possible in one minute) and 'visuospatial abilities' (indicating which two-dimensional shapes from a larger 
set are needed to exactly fill up a given space) were defined by subscales of the Groninger Intelligence Scale (GIT) [47]. 'Orientation', 'attention', 'praxis', and 'language' are subtests of the CAMCOG [39]. In addition to the 8 cognitive domains, we included also tests to assess 'mental speed', as this aspect may be of relevance according to previous studies [29, 56, 185]. 'Mental speed' was based on a combination of the scores of the first part of the Stroop Colour Word Test (SCWT) [45] with the scores on the first and second part of the Concept Shifting Test (CST) [82], time needed to complete these tests was the variable of interest. Definition of a cognitive deficit was described earlier [29, 49, 101].

\section{Psychiatric Symptoms}

The Symptom Checklist (SCL-90) was used to assess psychiatric symptoms. This questionnaire is a multi-dimensional self-rating scale and covers a spectrum of psychiatric symptoms: anxiety, agoraphobia, depression, symptoms of somatisation, interpersonal sensitivity (i.e. dissatisfaction with oneself in relation to others), insufficiency of thinking and acting (i.e. compelling thoughts and impulses), sleeping problems, and hostility [37]. We used the Dutch version of the SCL-90 [167]. This scale has been validated for patients with stroke [186]. Patients were asked to indicate, on a 5-point scale, how much hinder they had from certain psychiatric symptoms in the last week. The SCL-90 consists of 90 items with a maximum score of 450 and a range of 90 to 450, a higher score indicating more hinder from a psychiatric symptom. If a patient was not able to fill in the questionnaire by themselve the caregiver was asked to assist.

The cut-off scores for psychiatric symptoms in the normgroup were as follows: anxiety $=15$, agoraphobia $=9$, depression $=24$, symptoms of somatisation $=$ 19 , interpersonal sensitivity $=15$, insuffiencency of thinking and acting $=27$, sleeping problems $=6$ and hostility $=9$. A psychiatric symptom was considered present if stroke patients scored higher than the normative group mentioned in the manual of the Dutch version of the SCL-90 [167]. This normative group consists of 2368 healthy people who took part in a population survey. Ages ranged from 20 to 65 years (no mention of mean age), sex was equally distributed, and educational level was on average secondary school.

\section{Diagnosis vascular cognitive impairment}

Vascular Dementia: Dementia was diagnosed independently by an experienced neuropsychiatrist and a neuropsychologist applying the DSM IV criteria. Both clinicians are experts in the field of dementia. The diagnosis was based on all available data, including information about medical history, structured 
interview with an informant, clinical observation, and test performances. Interference with daily activities due to cognitive deficits was assessed by the Interview for Deterioration in Daily life in Dementia (IDDD) [38]. The diagnosis of $\mathrm{VaD}$ was based on the NINDS-AIREN criteria [24]. When there was a disagreement between the two clinicians, the final diagnosis was negative; this was the case in 5 patients. Agreement between the two clinicians was excellent $(\mathrm{K}=$ $0.88 ; \mathrm{p}=.00)$.

Post-Stroke MCI: Post-stroke MCI was diagnosed if patients fulfilled the following criteria: cognitive impairment on at least one of the cognitive domains; no interference of the cognitive impairments with daily activities, assessed by the IDDD; and no dementia. These criteria are largely based on the criteria for MCI [81]. This definition encompasses all categories as described in the recent proposal for subtyping MCI: MCI amnestic type, MCI multiple domain type, and single non memory type [81].

\section{Statistical Analysis}

Four types of analyses were performed. Firstly, baseline characteristics and frequency of psychiatric symptoms were analysed with descriptive statistics. Secondly, in order to investigate whether psychiatric symptoms increase the risk of either $\mathrm{VaD}$, or post-stroke $\mathrm{MCI}$, the possible association between baseline psychiatric symptoms and diagnosis of $\mathrm{VaD}$ or post-stroke $\mathrm{MCI}$ was analysed using odds ratios. Patients with $\mathrm{VaD}$ and patients with post-stroke $\mathrm{MCI}$, at baseline, were compared with patients without a cognitive disorder, these are cross -sectional analyses.

Thirdly, the extent to which psychiatric symptoms were predictive for subsequent cognitive decline was examined. Changes on CAMCOG functioning and performance on the cognitive domains memory, executive functioning, mental speed, visuospatial functioning and calculation, were used to determine whether psychiatric symptoms predicted changes in cognitive functioning. In a previous study among healthy volunteers an annual decline of 1.6 points on the CAMCOG was found. We defined cognitive decline on the CAMCOG as a 5-point decline compared to an earlier assessment. Decline on the specific cognitive domains was defined as a decrease of $1 \mathrm{SD}$ on the test performance measuring that domain, compared to an earlier assessment. As the relation between neuropsychological test performance and psychiatric symptoms is non-linear $\chi^{2}$, test were used. Changes in cognitive functioning from 1 to 6 months and from 6 to 24 months after stroke were determined, to investigate whether baseline psychiatric symptoms were related to cognitive changes directly after stroke and to cognitive changes later after stroke. In these analyses, the number of patients with psychiatric symptoms that showed improvement or deterioration 
was compared with the number of patients without psychiatric symptoms.

Fourthly, multiple regression analysis was performed to investigate the independent relation between psychiatric symptoms at baseline and cognitive performance at follow-up. In this analysis cognitive functioning was the dependent variable and psychiatric symptoms, age, baseline MMSE, side (leftright) and location (territorial-lacunar) of the stroke the independent variables. Missing data were imputed according to a standard procedure as described in Tabachnick et al. [51]. Data imputation was performed if there was at least one other test available within a specific cognitive domain and if the missing test was administered on an earlier or later measurement period.

\section{Results}

One hundred and ninety-six patients participated in the CODAS study (see table 1 for baseline characteristics). Ten patients (5.5\%) could not fill in the SCL90 due to severe cognitive deficits and 30 (15.3\%) refused assessment, therefore at baseline in 156 patients the SCL-90 was assessed.

\begin{tabular}{ll}
\hline Table 1: Baseline data & \\
\hline $\mathrm{N}$ & 156 \\
Education low/high & $84 / 72$ \\
Sex M/F & $86 / 70$ \\
Age mean (SD) & $67.7(12.4)$ \\
MMSE mean (SD) & $25.9(3.3)$ \\
Territorial-lacunar-haemorrhagic & $40.4 \%-51.3 \%-8.3 \%$ \\
Side: left-right-both & $37.7 \%-59.1 \%-3.2 \%$ \\
White matter lesions present & $19.8 \%$ \\
Silent infarcts present & $37.4 \%$ \\
\hline SD = Standard Deviation & \\
\hline
\end{tabular}

At 6 months after stroke 173 patients could be re-assessed; 4 patients had died, 14 refused further participation, 4 patients refused participation in this assessment, and 1 patient was untraceable. At 12 months after stroke 157 patients could be re-assessed; 8 patients had died (between the second and third assessment), 6 refused further participation, 4 patients refused participation in this assessment, and 2 patients were too ill. At 24 months after stroke 143 patients could be re-assessed; 15 had died (between the third and fourth assessment), 4 patients refused, and 1 patient was too ill. In all $73 \%$ of the original sample could 
be re-assessed after 2 years. Patients who could not be re-assessed did not differ from patients who were re-assessed in terms of baseline MMSE, age, education, or sex. At baseline 156 (79.6\%) subjects completed the SCL-90, 30 (15.3\%) patients refused, and $10(5.1 \%)$ patients could not fill in the questionnaire reliably due to too severe illness or cognitive handicaps. At 6, 12, and 24 months, 136 (78.6\%), 139 (85.4\%), and 119 (82.1\%) completed the questionnaire; 32 (18.5\%), $13(8.3 \%)$, and $18(12.4 \%)$ refused, and $4(2.3 \%), 7(4.6 \%)$, and $8(5.5 \%)$ had too severe physical or cognitive handicaps respectively. Not all patients completed the entire questionnaire, and thus information for some subscales is missing. If more than $50 \%$ of the items of a subscale were missing, the subscale score was not calculated. In other cases it was calculated as described in the manual of the SCL-90 [37]. Patients who did not complete the SCL-90 were similar to patients who did complete the SCL-90 with regard to age (mean age 71.0 (SD 12.6) versus 67.7 (SD 12.4) for patients who did not and those who did complete respectively), educational level (65\% versus $53.8 \%$ respectively had a low educational level), and site of the stroke (left hemisphere: $40.5 \%$ versus $43.0 \%$ respectively). Patients who did not complete the SCL-90 performed somewhat lower on the MMSE (mean 23.5, SD 3.8) compared to patients who completed the SCL-90 (mean 25.9, SD 3.3).

Table 2 presents data about the prevalence of psychiatric symptoms after stroke.

\begin{tabular}{lllll}
\hline \multicolumn{5}{c}{ Table 2: Number of people with psychiatric symptoms (score above cut-off) } \\
\hline \multicolumn{5}{l}{ N people with symptoms / N total (\%) } \\
& 1 month & 6 months & 12 months & 24 months \\
\hline Agoraphobia & $70 / 153(45.8)$ & $54 / 136(39.7)$ & $49 / 137(35.8)$ & $50 / 119(42.0)$ \\
Anxiety & $62 / 155(40.0)$ & $50 / 136(36.8)$ & $47 / 139(33.8)$ & $41 / 119(34.5)$ \\
Depression & $76 / 155(49.0)$ & $57 / 136(41.9)$ & $64 / 137(46.7)$ & $50 / 119(42.0)$ \\
Somatisation & $89 / 155(57.4)$ & $73 / 135(54.1)$ & $76 / 136(55.9)$ & $61 / 119(51.3)$ \\
Insufficiency & $88 / 156(56.4)$ & $77 / 134(57.4)$ & $75 / 139(55.1)$ & $69 / 119(57.9)$ \\
Sensitivity & $42 / 155(27.1)$ & $32 / 134(23.8)$ & $37 / 136(27.2)$ & $36 / 119(30.3)$ \\
Sleep & $70 / 156(44.9)$ & $52 / 136(38.2)$ & $58 / 137(42.0)$ & $42 / 119(35.3)$ \\
Hostility & $39 / 155(25.2)$ & $40 / 136(29.4)$ & $31 / 137(22.6)$ & $31 / 119(26.1)$ \\
Total Score & $86 / 155(55.5)$ & $68 / 134(50.7)$ & $73 / 135(54.1)$ & $57 / 119(47.9)$ \\
\hline
\end{tabular}

More than $50 \%$ of the patients reported psychiatric symptoms after stroke, and most often symptoms on the subscales depression, somatisation, and insuffi- 
ciency of thinking and acting.

In order to investigate the relation between baseline psychiatric symptoms and diagnosis $\mathrm{VCI}$, patients with either $\mathrm{VaD}$ or post-stroke $\mathrm{MCI}$ were compared with patients without cognitive deficits. At baseline, 15 (9.6\%) patients had VaD, $113(72.4 \%)$ had post-stroke MCI, and $28(17.9 \%)$ had no cognitive impairments. Table 3 presents data about the numbers and percentages of patients with a psychiatric symptom in each diagnostic group.

\begin{tabular}{|c|c|c|c|c|c|c|c|}
\hline \multirow[b]{2}{*}{ Symp } & \multicolumn{3}{|c|}{$\underline{\text { Vascular Dementia }}$} & \multicolumn{3}{|c|}{ Post stroke MCI } & \multirow{2}{*}{$\begin{array}{l}\text { No cogn } \\
\text { deficit } \\
\text { Nsymp/Ntot } \\
(\%)\end{array}$} \\
\hline & $\begin{array}{l}\text { Nsymp/ } \\
\text { Ntot (\%) }\end{array}$ & OR & $\mathrm{CI}$ & $\begin{array}{l}\text { Nsymp/ } \\
\text { Ntot (\%) }\end{array}$ & OR & $\mathrm{CI}$ & \\
\hline Agora $^{1}$ & $10 / 14(71.4)$ & 3.6 & $0.9-14.7$ & $49 / 112(43.8)$ & 1.1 & $.5-2.7$ & $11 / 27(40.7)$ \\
\hline Anxie $^{2}$ & 8/14 (571) & 2.1 & $0.6-7.6$ & $43 / 113(38.0)$ & 0.9 & $.4-2.7$ & $11 / 28(39.3)$ \\
\hline Depre $^{3}$ & $12 / 14(85.7)$ & 8.0 & $1.5-42.7^{*}$ & $52 / 113(46.0)$ & 1.1 & $.5-2.6$ & $12 / 28(42.9)$ \\
\hline Soma ${ }^{4}$ & $9 / 14(64.3)$ & 1.2 & $0.3-4.4$ & $63 / 113(55.8)$ & 0.8 & $.4-1.9$ & $17 / 28(60.7)$ \\
\hline Insuff $^{5}$ & $13 / 15(86.7)$ & 7.5 & $1.4-39.5^{*}$ & $62 / 113(54.9)$ & 1.4 & $.6-3.2$ & $13 / 28(46.4)$ \\
\hline Sensi ${ }^{6}$ & $7 / 14(50.0)$ & 3.0 & $0.8-11.6$ & $28 / 113(24.8)$ & 0.9 & $.4-2.6$ & $7 / 28(25.0)$ \\
\hline Hosti $^{7}$ & $7 / 14(50.0)$ & 3.0 & $0.8-11.6$ & $25 / 113(22.1)$ & 0.9 & $.3-2.2$ & $7 / 28(25.0)$ \\
\hline Sleep & $7 / 15(46.7)$ & 0.8 & $0.2-2.7$ & $48 / 113(42.5)$ & 0.7 & $.3-1.5$ & $15 / 28(53.5)$ \\
\hline Total & $12 / 14$ (85.7) & 6.9 & $1.3-36.8^{*}$ & $61 / 113(53.9)$ & 1.4 & $.6-3.1$ & $13 / 28(46.4)$ \\
\hline
\end{tabular}

Symp : symptoms ( ${ }^{1}$ agoraphobia, ${ }^{2}$ anxiety, ${ }^{3}$ depression, ${ }^{4}$ somatisation, ${ }^{5}$ insufficiency, ${ }^{7}$ sensitivity, ${ }^{8}$ hostility) | Nsymp / Ntot: Number of patients with psychiatric symptoms | Total number of patients who filled in the subscale | CI: confidence interval $\mid{ }^{*} \mathrm{p}<0.05$

Patients diagnosed with VCI after they had had a stroke had many psychiatric complaints, but only the presence of depression, insufficiency of thinking and acting, and total score on the SCL-90 increased the risk of being diagnosed with VaD.

Patients with psychiatric symptoms were compared to patients without these psychiatric symptoms with regard to the course of cognitive functioning. Patients with psychiatric symptoms declined more often on executive functio$\operatorname{ning}\left(\mathrm{x}^{2}=5.1 \mathrm{df}=2, \mathrm{p}=.08\right)$ and on the CAMCOG $\left(\mathrm{X}^{2}=5.7 \mathrm{df}=2, \mathrm{p}=.049\right)$ from 1 month to 6 months after stroke, and on the test that measure mental speed from 6 to 24 months after stroke $\left(x^{2}=5.9 \mathrm{df}=2, p=.05\right)$, however, these differences 
are not very robust. On the other cognitive domains, the course of cognitive functioning was comparable between patients with psychiatric symptoms and those without these psychiatric symptoms. Table 4 presence data of cognitive decline on the CAMCOG.

Table 4: Comparison of patients with psychiatric symptoms to patients without, with regard to cognitive decline on the CAMCOG.

\begin{tabular}{|c|c|c|c|c|c|c|c|c|}
\hline \multirow[b]{3}{*}{ Agoraphobia } & \multirow{2}{*}{\multicolumn{2}{|c|}{$\begin{array}{l}1 \text { to } 6 \text { months } \\
\text { N Decl. } \\
\text { Psych.+ / } \\
\text { Npsych }+(\%) \ddagger\end{array}$}} & & & \multicolumn{4}{|c|}{$\underline{6 \text { to } 24 \text { months }}$} \\
\hline & & & \multicolumn{2}{|c|}{$\begin{array}{l}\text { N Decl. Psych.- } \\
\text { / Npsych } \\
-(\%) \dagger\end{array}$} & \multicolumn{2}{|c|}{$\begin{array}{l}\text { N Decl. Psych. } \\
+/ \text { Npsych }+ \\
(\%) \ddagger\end{array}$} & \multicolumn{2}{|c|}{$\begin{array}{l}\text { N Decl. } \\
\text { Psych.- / } \\
\text { Npsych - (\%)† }\end{array}$} \\
\hline & $4 / 21$ & $(19.1)$ & $1 / 28$ & $(3.6)$ & $9 / 27$ & $(33.3)$ & $7 / 15$ & $(46.7)$ \\
\hline Anxiety & $4 / 17$ & (23.5) & $1 / 33$ & $(0.3)^{*}$ & $5 / 18$ & (27.8) & $11 / 18$ & (61.1) \\
\hline Depression & $5 / 25$ & (20.0) & $0 / 25$ & $(0.0)^{*}$ & $8 / 24$ & (33.3) & $8 / 18$ & $(44.4)$ \\
\hline Somatisation & $4 / 24$ & (16.7) & $1 / 26$ & $(3.8)$ & $7 / 25$ & (28.0) & $9 / 17$ & (52.9) \\
\hline Insufficiency & $5 / 30$ & (16.7) & $0 / 20$ & $(0.0)^{*}$ & $8 / 27$ & (29.3) & $7 / 15$ & $(46.7)$ \\
\hline Sensitivity & $1 / 9$ & (11.1) & $4 / 41$ & $(9.8)$ & $5 / 12$ & $(41.7)$ & $11 / 30$ & (36.7) \\
\hline Hostility & $0 / 8$ & $(0.0)$ & $5 / 42$ & (11.9) & $2 / 10$ & (20.0) & $14 / 32$ & $(43.8)$ \\
\hline Sleep & $2 / 17$ & (11.8) & $3 / 33$ & $(9.1)$ & $7 / 21$ & (33.3) & $12 / 21$ & (57.1) \\
\hline Total & $5 / 26$ & 19.2 & $0 / 24$ & $0.0^{*}$ & $8 / 26$ & 30.8 & $8 / 16$ & 50.0 \\
\hline \multicolumn{9}{|c|}{$\begin{array}{l}{ }^{*} \mathrm{p}<.05 \mid \text { Decl: decline } \mid \ddagger \text { : Number of patients with psychiatric symptoms that } \\
\text { declined/Number of patients with psychiatric symptoms | } \dagger \text { : Number of patients } \\
\text { without psychiatric symptoms that declined / Number of patients without psychi- } \\
\text { atric symptoms }\end{array}$} \\
\hline
\end{tabular}

The presence of psychiatric symptoms at baseline influenced whether patients showed cognitive changes between 1 and 6 months after stroke. Patients with psychiatric symptoms had a higher chance of cognitive deterioration, measured with the CAMCOG, than did patients without such symptoms. This relation was significant for anxiety, depression, insufficiency of thinking and acting, and total score of the SCL-90. The presence of psychiatric symptoms at baseline was not associated with cognitive changes between 6 and 24 months after stroke. Patients with complaints of anxiety or depression or who had overall psychiatric symptoms decline more often compared to patients without psychiatric complaints. Patients with psychiatric symptoms decline with a mean of 10.1 (SD 6.6) points on the CAMCOG from 1 to 6 months, whereas patients without psychiatric symptoms decline with a mean of 7.8 (SD 3.3) points. From 6 to 24 months this decline is lower (2.8 points form the patients with psychiatric symp- 
toms and 0.3 points for the patients without psychiatric symptoms).

The incident cases of $\mathrm{VaD}$ or $\mathrm{MCI}$ were too few to perform valid multiple regression analyses. There were 7 incident $\mathrm{VaD}$ cases ( 1 at 6 months after stroke, 1 at 12 months, and 5 at 24 months after stroke). There were 5 incident MCI cases ( 1 at 6 months after stroke, 3 at 12 months and 1 and 24 months after stroke). Therefore, we could only perform analyses in which we compared patients who declined on cognitive performance (assessed with the CAMCOG) to those who improved, and found that patients who declined had more often a territorial stroke $(28.8 \%$ of the patients who declined had a territorial infarct, whereas only $3.8 \%$ of the patients who improved; $\chi^{2}=5.9, \mathrm{df}=1, \mathrm{p}=.02$ ). We also compared patients with psychiatric symptoms to those without these symptoms and found that patients with psychiatric symptoms have lower baseline MMSE-scores (mean 25.3, SD 3.5) compared to patients without psychiatric symptoms (mean 26.7, SD 2.9) (t $=2.5, \mathrm{df}=153, \mathrm{p}=.01$ ).

\section{Discussion}

In this study we investigated whether stroke-patients with psychiatric symptoms had more cognitive decline compared to patients without psychiatric symptoms. More than $50 \%$ of the stroke patients reported psychiatric symptoms up to 2 years after the event. Psychiatric symptoms were most prevalent among patients with a post-stroke diagnosis of $\mathrm{VaD}, 85 \%$ of the patients with $\mathrm{VaD}$ reported psychiatric symptoms. The presence of psychiatric symptoms, had predictive value for the decline in executive functioning, mental speed, and functioning on the CAMCOG, at 6 months after stroke.

This is the first study that investiagted a broad range of psychiatric symptoms after stroke. Most of the psychiatric symptoms reported concerned somatisation and insufficiency of thinking and acting, which is perhaps to be expected in people with physical handicaps and cognitive deficits due to a stroke. However, depressive symptoms were also common, as has been reported by others [173176, 187].

Patients who were, at baseline diagnosed with VaD reported, compared to patients with $\mathrm{MCI}$ or no cognitive deficits more often psychiatric symptoms ( $85.7 \%$ compared to $53.9 \%$, and $46.4 \%$ respectively). Although on the total SCL90 scale more patients with MCI reported psychiatric symptoms compared to patients without cognitive deficits, this was not found for all subscales of the SCL-90. The absence of an association between psychiatric deficits and poststroke $\mathrm{MCI}$ could be related to the fact that the concept $\mathrm{MCI}$ is heterogeneous. However, as none of the patients with MCI had interference with daily life due to their cognitive deficits, we believe our group is homogeneous.

Incident case of $\mathrm{VaD}$ and $\mathrm{MCI}$ were too few to perform valid multiple regres- 
sion analysis, therefore in our study we could not define whether psychiatric symptoms can independently predict cognitive decline. We found differences with regard to demographic and stroke related variables between patients with $\mathrm{VaD}$ and patients with $\mathrm{MCI}$ and also between patients who declined and those who did not. In future research the independent contribution of these variables to deterioration on cognitive functioning after stroke should be investigated.

Patients with psychiatric symptoms, especially depression, anxiety, and insufficiency of thinking and acting were at increased risk of cognitive deterioration. However, it should be mentioned that 'insufficiency of thinking and acting' has cognitive components in it and as such an association between this subscale and cognition is not remarkable. Other studies also found that depression influenced cognitive performance after stroke [129, 177-180]. Depression and anxiety can be grouped together as symptoms of 'affect', and thus it is possible that people with affective disorders are at increased risk of developing cognitive deficits after stroke.

The relation between cognitive deficits and psychiatric symptoms could be 3fold. First psychiatric symptoms may cause cognitive deficits. Second, cognitive deficits may give rise to psychiatric symptoms, or lastly both psychiatric symptoms and cognitive deficits are occurring independently. Although no firm conclusions can be drawn, our results suggest that psychiatric symptoms cause cognitive decline. In an other study of our group with a similar cohort, cognitive deficits at baseline did not predict the development of psychiatric disorders [224].

In an earlier study we found that $\mathrm{VaD}$ was related to the presence of a territorial infarction [100]. Robinson et al. found that long-lasting depression after stroke is related to cortical lesions mostly left sided [173, 174, 177, 188, 189]. We found that more patients with $\mathrm{VaD}$ than patients with post-stroke $\mathrm{MCI}$ or patients without cognitive deficits had left-sided stroke, but the difference was not statistically significant. Moreover, in the study of Robertson et al. patients with psychiatric symptoms more often had left-sided lesions, and patients whose cognitive function improved more often had right-sided lesions as has been found earlier [123]. Thus it is possible that the interaction between psychiatric symptoms and $\mathrm{VCI}$ is related to a common underlying pathophysiological mechanism, such as left-sided territorial lesions. Future research should address this issue. The low evidence for this hypothesis in our results could be related to the fact that patients with severe aphasia were excluded. If these patients were included, more patients with left hemisphere stroke were probably included. Future research should address this issue. We only found an association between psychiatric symptoms at baseline and cognitive decline between 1 and 6 months after stroke and not between 6 and 24 months. This indicates that other factors like older age may be of more influence later after stroke than are psychiatric symptoms. Moreover, most cognitive changes occur directly after stroke. These 
factors could be of influence on the low association between cognitive decline and psychiatric symptoms later after stroke.

Our study had some shortcomings. Firstly, we used a questionnaire to assess psychiatric symptoms; we did not diagnose neuropsychiatric disorders by psychiatric clinical assessment. However, our results indicate that psychiatric symptoms are related to cognitive deficits. Secondly, to define whether a psychiatric symptom was present, we had to rely on the normative data described in the manual of the SCL-90. These data are for slightly younger individuals, and thus prevalence rates might be overestimated. However, in an earlier study of our group a high validity for the subscale depression in diagnosing depression in a stroke population was reported [186]. Thirdly, only a few patients became demented. This is because only patients with MMSE score $>15$ were included, as such excluding patients with very severe cognitive deficits. However, even with our small number of patients we found that patients with psychiatric symptoms were at higher risk of $\mathrm{VaD}$ than were patients without psychiatric symptoms. Fourthly, some attrition occurred. However, there were no differences between patients who did or did not completed the SCL-90 in terms of educational level, sex and age, although the patients who did not complete the questionnaire had a lower mean MMSE-score. Our results would have been more robust if these people would have participated.

In conclusion, we found that psychiatric symptoms are common after stroke and that these symptoms are prevalent in patients with severe cognitive deficits. Future research should focus on a broad spectrum of psychiatric disorders to find out how these influence cognitive function after stroke. 


\section{XIII}

General Discussion 
Dissatisfaction with the concept vascular dementia $(\mathrm{VaD})$ led to the introduction of a broader concept, Vascular Cognitive Impairment (VCI). VCI refers to all forms of mild to severe cognitive impairment associated with, and presumably caused by, cerebrovascular disease. Formal criteria for the diagnosis of $\mathrm{VCI}$ are still not fully defined, and little is known about possible risk factors or its course. $\mathrm{VCI}$ is not a single condition, but instead it encompasses a range of changes and clinical manifestations, and there is no single diagnostic feature. The phenomenology of VCI has scarcely been studied.

The aim of the CODAS study was to investigate VCI after stroke. The phenomenology, course, and risk factors of cognitive disorders after stroke were studied in 196 patients who were followed up for 2 years after stroke. Clinical assessment was performed at 1, 6, 12, and 24 months after stroke. Results from the CODAS project are discussed below.

\section{Main findings of this thesis}

\section{What is the phenomenology of VCI?}

Cognitive deficits are common after stroke. We found that more than $80.0 \%$ of the patients had VCI: $90 \%$ had mild cognitive impairment (MCI) and 10\% had $\mathrm{VaD}$ (chapter IV \& V). The prevalence of $\mathrm{VaD}$ varied according to the diagnostic systems used (Chapter VI).

Most studies have investigated cognitive impairment 3 months after stroke and have focused on the development of $\mathrm{VaD}$ [2-5]. In the CODAS project, we focused on VCI from 1 month after stroke onwards, and as such we were able to identify patients with mild cognitive deficits, deficits which have often not been detected in other studies. The high prevalence of VCI after stroke indicates that cognitive deficits are a major problem. Cognitive impairments limit a person's functional ability and lowered his/her quality of life [116, 190-195], and should be taken into consideration when describing and/or predicting a patient's level of independence. However, up to now the phenomenology of VCI is unclear. Few studies investigated the neuropsychological profile of VCI and found that it was characterised by impairments of attention and executive function, with slowing of motor performance and information processing [137]. However, because VCI includes diverse syndromes with different causes, the striking differences in clinical presentation and course between its different forms are not surprising. Similar to earlier studies, we found that memory function was not as severely disturbed after stroke as is often assumed in clinical practice. In contrast, mental speed was the cognitive functioning most often disturbed after stroke $[56,185]$ (Chapter IV \& V). So, the typical cognitive profile of patients with 
$\mathrm{VCI}$ is characterised by mental slowness -patients had not only problems with tasks that measure mental speed, but also with tasks that need to be performed under time pressure, such as the calculation task.

In addition to cognitive disorders, more than $50 \%$ of the stroke patients reported psychiatric symptoms. This high prevalence accords with other studies of psychiatric disorders after stroke [173-175, 187, 189, 196]. The presence of depression or anxiety predicts a poor functional outcome after stroke [174, 180, 197, 198]. In most stroke studies, psychiatric symptoms have been investigated separately form cognitive deficits [173-176, 187]; however, we found an association between psychiatric symptoms and cognitive deficits (Chapter XI \& XII), with the prevalence of psychiatric symptoms being highest in patients with VCI.

The Checklist for Cognitive and Emotional consequences following stroke (CLCE-24) is suitable for detecting both cognitive and emotional problems after stroke (Chapter XI). Stroke patients who reported cognitive or emotional complaints on this checklist performed worse on cognitive tasks.

\section{What is the course of VCI?}

While the cognitive deficits and psychiatric symptoms of most patients of our cohort remained stable, in some they deteriorated and in others they improved (Chapter IV,V \& XII).

On average, patients showed a 2.5 points improvement on the CAMCOG 2 years after stroke ( $26.9 \%$ deteriorated and $64.8 \%$ improved at least 1 point). If recovery was defined in terms of complete remission of cognitive impairment, which has never been studied in a stroke population, we found that $20 \%$ of the patients recovered (Chapter VIII). It is important to identify patients who recover from $\mathrm{MCI}$ because in general doctors hold the pessimistic view that cognitive function will only get worse in patients with VCI. In the literature, improvement of cognitive performance after stroke has also been reported in up to $50 \%$ of stroke patients $[15,89,91,113,123-125]$. In the CODAS project, recovery of cognitive function was associated with female sex and higher cognitive functioning at baseline. Moreover, women and patients with higher cognitive functioning at baseline had fewer stroke risk factors than did men (29.1\% versus $34.4 \%)$ and patients with lower cognitive functioning at baseline (28.4\% and $42.9 \%)$. It can thus be hypothesised that the brains of patients with one or more stroke risk factors are less able to adapt to the consequences of stroke because they may have a lower brain reserve capacity. The relation between brain reserve capacity and/or brain adaptation and cognitive recovery after stroke should be investigated. Although most research suggests that the cognitive function of patients with VaD deteriorates [159, 199-206], in our series cognitive function improved 
in 1 of 3 patients (Chapter V). This suggests that recovery from post-stroke dementia is possible. However, our finding of cognitive improvement may be associated with two other aspects. First, the absence of memory deficit at later assessments may have led to patients being classified as being non-demented. Second, criterion B of the DSM IV (i.e. the cognitive deficits each cause significant impairment in social or occupational functioning) is difficult to apply in a stroke population, in which interference with daily life is mostly due to physical handicap. Consequently, relatives of patients could have misinterpret the question about interference in daily life due to cognitive deficits, which could have led to an overemphasize of the prevalence of recovery from post-stroke dementia.

Another aspect regarding the course of changes in cognitive function is the prodromal stage of dementia. We investigated whether MCI, which is traditionally related to the predementia stage of Alzheimer's disease (AD), has predictive value for the development of $\mathrm{VaD}$. The subtype 'MCI multiple domains impaired' had high sensitivity, but low predictive value for the development of VaD 2 years later (Chapter VII). This means that this subtype identifies patients in the predementia stage of $\mathrm{VaD}$. However, the diagnosis 'MCI multiple domains impaired' has low predictive value for the development of $\mathrm{VaD}$. This finding should be interpreted with caution, because only five patients became demented. Larger studies are needed to investigate the predictive value of different MCI subtypes for dementia. More than $50 \%$ of the stroke patients with $\mathrm{MCI}$ were diagnosed with the subtype 'MCI multiple domains impaired memory -'. This indicates there is a predementia stage in $\mathrm{VaD}$, as there is in AD. It is important to identify patients at risk of dementia because it make timely intervention possible, perhaps even preventing the onset of the dementing process. Moreover, knowledge of the risk of dementia may enable patients and their relatives to take steps to prolong the patients' autonomy, which may lessen feelings of distress or helplessness among both patients and their relatives. Memory impairment is not as important in $\mathrm{VaD}$ as it is in the prodromal stage of $\mathrm{AD}$. Thus the traditional concept 'amnestic $\mathrm{MCI}^{\prime}$ is of less use in patients with vascular pathology. In the CODAS project, most cases of post-stroke dementia were diagnosed within 1 month of the stroke event. This confirms the traditional concept of an abrupt onset of $\mathrm{VaD}$. However, 10 incident $\mathrm{VaD}$ cases were identified, and all patients were diagnosed multiple domains MCI at earlier assessments. Thus the failure to develop dementia in the acute phase after stroke does not imply that VaD will never develop. We could not verify that a predementia stage is found only in a subgroup of patients with small vessel disease, as suggested by Meyer et al [207] . 


\section{Which risk factors can be identified in the development of VCI?}

Stroke increases the risk of dementia, and also the risk of milder cognitive impairments. We found that the presence of a territorial infarct, besides older age and lower level of education increased the risk of VCI (Chapter IX). A unique facet of CODAS is that VCI was defined in terms of both vascular MCI and $\mathrm{VaD}$. In earlier studies, only $\mathrm{VaD}$ and $\mathrm{VCI}$ were investigated as separate entities. A possible reason why we did not find, unlike earlier studies [17, 62, $86,133,134]$, other brain-related risk factors such as white mater lesions or silent infarcts is that few of the CODAS participants had such lesions, possible because we excluded patients with severe cognitive deficits. Cognitive deficits are related to white matter lesions and silent infarcts [69, 92, 208, 209]. Nevertheless, we found small vessel disease (either white matter lesions or silent infarcts or both) was associated with an increased risk of VCI later after stroke.

As small vessel disease progresses over time [54], we investigated the relation between progression of these lesions and cognitive performance. Patients with progressive brain damage, as assessed on repeat CT-scan 2 years after stroke, performed worse on cognitive tasks, than patients without progressive brain damage, yet, the prognosis of cognitive functioning was similar (Chapter X). In contrast to an earlier study [156], we did not find a relation between progressive vascular brain damage on $\mathrm{CT}$ and decline in cognitive function. This may be because cognitive deterioration becomes manifest only when the extent of ischemic damage in the deep structures has passed a certain critical threshold. The high frequency of slowing of mental speed can be explained by the synergistic way ischemic lesions act to impair cognition, a phenomenon that may be explained in terms of global damage to a neural network [210]. This can be contrasted to a more focal type of damage, such as the hippocampal damage leading to amnesia. In the CODAS study, more than $50 \%$ of the patients had lacunar lesions, which often co-occur with small vessel disease [211]. This in turn affects the main pathways in the neural network.

Besides brain damage, psychiatric symptoms were also associated with the development of cognitive deficits after stroke. Most investigators agree that psychiatric symptoms cause cognitive deficits, and that these deficits may mimic those of dementia, giving rise to the concept of 'dementia of depression' $[129,177-180,212,213]$. In the CODAS cohort we could not investigate whether psychiatric symptoms were risk factors for $\mathrm{VCI}$ as at the second and later assessments there were too few incident cases. However, we did find that patients with psychiatric symptoms were at increased risk of developing $\mathrm{VaD}$ of cognitive decline (defined as 5-point lower score on the CAMCOG) (Chapter XII). Thus, psychiatric symptoms, mostly depression, somatisation, and insufficiency of thinking and acting appear to be risk factors for VCI. 


\section{Methodological considerations}

The study had some limitations that should be mentioned before the implications of our findings can be discussed. Firstly, the patients were tested four times, which could have led to a learning effect, and as such cognitive improvement may not have been due to recovery per se. This effect can not be ruled out and is more likely for some tests. For instance, learning effects are reported to be minimal for the Stroop and Concept Shifting Test (CST) [93]. We used parallel versions of the Auditory Verbal Learning Test (AVLT) to avoid a learning effect [93].

Secondly, we categorized the tests as assessing certain cognitive domains. Although this is a clinically relevant approach and also leads to data reduction, it remains slightly arbitrary. However, the reliability of the compound scores, which are elaborated in the method section, was excellent, except for executive functioning.

Lastly, attrition may have led to underestimation of VCI [214]. Although the rate of attrition was lower than that of other studies [86, 123, 215-217], patients who were lost to follow-up were older, had a lower educational level, and had lower MMSE scores. They may have been more likely to develop VCI, and thus their inclusion in the study might have led to more robust conclusions.

Despite the limitations, we believe that our results are representative of people coming to a stroke unit, and thus our results can be generalized to other stroke populations, without very severe cognitive deficits or with first-ever stroke.

An advantage of our design is that we examined the patients within 1 month after stroke, whereas most other studies investigated patienst 3 months after stroke $[2,6,16-18,62,89,91,139,140,143,198,218]$. We thus were able to identify patients at risk of $\mathrm{VCI}$ in an early stage after stroke. However, testing in the acute phase after stroke may have led to the false identification of cognitive deficits, because patients may not have been in a stable condition. Despite this confounding factor, one of the main aims of our study was to investigate how cognitive function changes in the early phase after stroke. Moreover, early neuropsychological testing even within 20 days after stroke evaluation, has been shown to provide valid prognostic information [166].

We excluded patients with severe cognitive deficits $(\mathrm{N}=46 ; 7.7 \%$ of the total stroke population), using an MMSE score $<15$ as cut-off. A score lower than 24 is commonly used as indicator of dementia [50,219]. Thus we excluded patients with very severe cognitive deficits, but included patients with mild to moderate dementia. Moreover, given our interest in the prodromal stage of $\mathrm{VaD}$, we thought that patients with severe aphasia or other sever cognitive deficits severe cognitive deficits were not be able to undergo extensive neuropsychological testing. Consequently, we studied cognitive decline in those patients for whom 
such decline might be most relevant, namely patients without any prior severe cognitive problems. Another strong point of the methodology was the relatively long study follow-up during which the patients underwent neuropsychological testing on a number of occasions. Most other studies only investigated $\mathrm{VCI}$ in a cross-sectional design or monitored the patients for up to 12 months after stroke $[2,6,16-18,62,89,91,139,140,143,198,218]$. Follow-up is needed to identify patients at risk of cognitive deterioration and to assess causal relations.

\section{Clinical implications}

The high prevalence of cognitive deficits shows that clinicians should be alert to cognitive deficits after stroke. They should not concentrate solely on memory deficits but also on mental speed, the most common cognitive deficit detected in this study. Moreover, clinicians should be aware that problems with mental speed give rise to memory problems. Memory problems due to mental slowness are probably easier to cope with than those due to pure amnesia.

Mild cognitive impairments could be a precursor of $\mathrm{VaD}$, just as they are in $\mathrm{AD}$ [207]. In the MCI study of CODAS (Chapter VII), all patients with VaD had been diagnosed with 'MCI multiple domains slightly impaired' 2 years earlier. This indicates that patients with deficits in multiple domains are at increased risk of developing dementia. Thus the diagnosis of VCI should not be based on specific cognitive problem but instead on the presence of more than one cognitive deficit.

We recommend that the cognitive function of patients be evaluated in the acute phase after stroke. The MMSE or CAMCOG are not appropriate instruments in this context because they do not include mental speed items and are often biased toward language and memory domains [181]. The CLCE-24 is an appropriate instrument to screen for both cognitive complaints and emotional symptoms.

Patients are more likely to come to the attention of medical services once symptoms of cognitive impairment become overt with the development of dementia, which is too late for preventive purposes [220]. Identification of $\mathrm{VCI}$ in an early stage after cerebrovascular damage serves two purposes. Firstly, to identify patients at risk of further cognitive decline in whom preventive treatment should be started. Secondly, to inform patients about their cognitive deficits in an early stage so that they can adapt their daily routine immediately and not wait until severe problems arise. In order not to miss patients with VCI, and consequently run the risk that their condition deteriorates unnecessarily, criteria need to be sensitive rather than specific; the ADDTC meets these requirements [103]. However, a high sensitivity may yield a high rate of false positives findings, and for this reason a diagnosis should be applied for detection purposes and not in telling a stigmatising diagnosis like $\mathrm{VaD}$. Because patients 
that perform on cognitive tasks relatively well may recover spontaneously, it is important that these patients be informed about the possibility of this favourable prognosis. Older stroke patients with a lower educational level and with territorially located infarctions are at increased risk of VCI.

\section{Conceptual and scientific considerations}

The finding that memory is not as severely disturbed after stroke as is often assumed, combined with the finding that memory impairment is not characteristic for the predementia stage of $\mathrm{VaD}$, has implications for the diagnosis $\mathrm{VaD}$. The traditional concept of dementia is based on the phenomenology of $\mathrm{AD}$ in, which a memory deficit is required. However, given the low prevalence of memory deficit after stroke, this traditional definition of dementia has to be replaced by a definition that includes a broader spectrum of cognitive deficits. Criteria for VaD should specify that dementia due to cerebrovascular disease should be defined in terms of cognitive deficits in at least two different cognitive domains, can sign interference of daily life activities.

The concept VCI covers a continuum of cognitive deficits from very mild cognitive deficits to dementia caused by vascular brain damage [7]. This concept draws the separate nosological concepts together, making early identification of cognitive deficits possible, which in turn facilitates preventive interventions. The chance of being able to prevent further cognitive decline is higher in patients with cerebrovascular disease than in patients with degenerative brain damages $[7,181,222]$, and consequently early identification is desirable. However, because there is no standard treatment for $\mathrm{VCI}$ and because little is known about primary and secondary prevention, studies directed to distinct causal and pathological factors (eg vascular and AD burden of the brain) are needed to pave the way for therapeutic progress. The clinical effectiveness of anti-hypertensive and antiatherosclerotic agents has not yet been established [137]. Most studies focussing on the prevention of $\mathrm{VCI}$ have concentrated on lowering the risk factors for stroke. For instance, in the an earlier study it was found that lowering blood pressure reduces the risk of dementia [221-223]. Moreover, cholinesterase inhibitors could be used to treat VCI. Reducing the risk of recurrent stroke, reduces the risk of developing dementia or cognitive decline [222, 223]. These findings support the importance of early identification of VCI.

Criteria for VCI have not been established yet. VCI encompasses a variety of changes within the brain with different causes and clinical manifestations and as such there is no single diagnostic feature. Consequently, subgroups of VCI should be formulated based on the underlying pathology, i.e. large artery disease, small vessel disease, cardiac embolic phenomena, and hemodynamic mechanisms. This subdivision of VCI into aetiological subtypes may 
be important, because it is the cause that requires treatment. Vascular damage is usually defined in terms of damage due to stroke, and other pathological mechanisms, such as small vessel disease, have received too little attention in the past. Consequently, little is known about the aetiology and course of different subtypes of $\mathrm{VaD}$.

There will always be the dilemma of how much influence neurodegenerative brain pathology (due to AD) has on the cognitive function of a person with VaD. The influence of neurodegenreative processes can only be excluded by carrying out neuropathological studies. However, because in CODAS most patients had a stable cognitive function and even $20 \%$ of the patients with MCI recovered, the likelihood that patients with mixed dementia were included was low. Moreover, dementia before stroke was an exclusion criterion for the studies described in chapter V, VI, VII, VIII, IX, and X.

The prevalence of psychiatric symptoms after stroke was high and, moreover, these symptoms were related to cognitive deterioration. In earlier studies, cognition was studied separately from psychiatric disorders. However, we found that psychiatric symptoms often co-occur with cognitive deficits after stroke, a fin-ding which is underexposed, in the current literature. The exact relation between the two is complex and may be explained in various ways. An underlying vascular component may be responsible for both psychiatric symptoms and cognitive deficits. Alternatively, both cognitive and psychiatric deficits could be caused by the stress that is related to an event such as stroke or cognitive deficits may be caused by psychiatric symptoms. Our results point to the last explanation because the patients with psychiatric symptoms were at increased risk of being diagnosed with $\mathrm{VaD}$ or of experiencing a deterioration. Moreover, in an earlier study of our group the hypothesis whether cognitive deficits led to depression was weakened as there was no such relation found [224].

Although the VCI is conceptually advantageous, the difficulty of diagnosing cognitive deficits after stroke remains. VCI is a heterogeneous concept with different vascular aetiologies causing different cognitive deficits. This may lead to the somewhat artificial definition of subgroups of VCI. Moreover, should cognitive deficit be defined in terms of deterioration compared to an earlier assessment or compared to a norm group? Both for clinical practice and research purpose, clearly defined criteria should be formulated because of comparability. Lastly, the concept VCI does not have prognostic validity it only identifies patients at risk of cognitive deterioration. These shortcomings can be attributed to the heterogeneity of the concept [225]. Based on the CODAS data, a diagnosis of VCI does not imply cognitive deterioration. We found that $20 \%$ of the patients with VCI recovered from their cognitive deficits (Chapter IV, V \& VIII). As such, the concept VCI should not be narrowed to cognitive deterioration, but also state that cognitive improvement is possible. 


\section{Future direction}

In future research, it may be possible to identify subgroups of VCI based on the different underlying pathogenetic mechanisms. This requires studies that includes patients with different types of cerebrovascular disease, in addition to stroke. Ideally, VCI should be studied in patients at risk for VCI: older patients, and patients with risk factors for cerebrovascular disease such as hypertension, diabetes, or cholesterol. Moreover, patients should be followed-up for longer periods in order to study their outcome and prognosis, to determine whether these differ depending on the aetiology of VCI. This information, combined with the observations from CODAS, should be used as supportive evidence for defining criteria for VCI. As such, therapies based on different aetiologies could be developed and evaluated.

A major issue that awaits clarification is the load or burden of vascular changes that is required to produce cognitive impairment. This is important in relation to VCI and for determining the role of vascular lesions in other disorders such as $\mathrm{AD}$ and depression. However, the location of the vascular lesion is important, and methods that can integrate lesion volume and location as causes of VCI need to be established. 
Summary 
Stroke is, after myocardial infarction and cancer, the third leading cause of death in Western countries and is associated with a high level of permanent handicap in the elderly. For patients who survive, stroke can have tremendous consequences, both physically and mentally. It is only recently that the cognitive deficits due to stroke have received more attention. Most studies on the relation between stroke and cognitive disorders have reported on vascular dementia $(\mathrm{VaD})$. However, the concept $\mathrm{VaD}$ has been questioned because it falsely implies a homogeneous entity with a single underlying pathology. The broader concept of vascular cognitive impairment (VCI) circumvents these problems [7]. In VCI, vascular refers to all causes of ischemic cerebrovascuar disease, while cognitive impairment encompasses all levels of cognitive decline. However, it is unclear how the cognitive deficits progress with time and which risk factors cause deterioration. More needs to be learned about these gaps in our knowledge before criteria for VCI can be defined and used to identify patients at risk of cognitive deficits or of becoming demented. The CODAS (COgnitive Disorders After Stroke) study is described in this thesis.

In chapter II we described the concept Vascular Cognitive Impairment (VCI). The term Vascular Dementia (VaD) is widely used, but it remains problematic. Hitherto, the term 'vascular' has been unclearly described. Most criteria for VaD insufficiently reflect the underlying pathology. The diagnosis 'dementia' from $\mathrm{VaD}$ is based on the criteria of Alzheimer's Disease. Consequently, in the diagnostic process of $\mathrm{VaD}$, the importance of memory disturbances has been overemphasised. The broader concept 'vascular cognitive impairment' (VCI) was recently introduced by Bowler and Hachinski [7] to describe a profile of cognitive deficits related to vascular brain damage. VCI includes not only dementia, but also milder forms of cognitive deterioration.

In chapter III the methodological set-up used in the CODAS project was described. This chapter comments on the methodological choice made in CODAS i.e. design, procedure, patient population, clinical assessment (both neuropsycholigical as medical assessment), and how we dealt with missing data. Moreover, the different diagnostic criteria used; post stroke mild cognitive impairment, $\mathrm{VaD}$, and how we defined cognitive improvement or deterioration are explained in this chapter.

In chapter IV we investigated memory functioning, executive functioning, mental speed performance, and overall cognitive functioning after stroke. Stroke patients $(\mathrm{N}=101)$ were compared with a healthy control group matched for age, sex, and level of education. Most cognitive deficits were found in the domain of mental speed ( $50 \%$ of the patients). Most people remained stable on the four cognitive domains (ranging from $37.6 \%$ to $83.5 \%$ ), and a substantial 
group improved (ranging from $12.9 \%$ to $52.1 \%$ ). Older and female patients had more cognitive disturbances. Overall the conclusion is that the prognosis of cognitive functioning after stroke is general favourable, especially in younger patients.

Long-term cognitive performance after stroke was described in chapter $V$. We investigated the frequency of post-stroke dementia, post-stroke Mild Cognitive Impairment (MCI), and post-stroke amnestic $\mathrm{MCI}$ at different times. In addition, cognitive disorders and their course were studied. Frequency of post-stroke dementia was about $10 \%$ at all the evaluation times. Most patients had poststroke MCI. Performance on almost all cognitive tests was improved 6 and 12 months after stroke. Thus, while the frequency of post-stroke dementia is low, the frequency of post-stroke $\mathrm{MCI}$ is high but improvement of cognitive function is possible.

In chapter VI we investigated the influence of different diagnostic criteria on the prevalence and incidence of post-stroke dementia. At 1, 6,12, and 24 months after stroke we clinically evaluated 194 first-ever stroke patients, without prestroke dementia. VaD was defined according to DSM-III, DSM-III-R, DSM-IV, ICD-10-NA, NINDS-AIREN, and ADDTC criteria. The prevalence of VaD was highest at 1 month; ranging from $11.3 \%$ with the NINDS-AIREN to $20.1 \%$ with the ICD-10-NA. The incidence was highest at 6 months, ranging from $2.6 \%$ with the ADDTC to $5.2 \%$ with the ICD-10-NA. We concluded that both the prevalence and incidence of $\mathrm{VaD}$ are highest directly after stroke but exact rates are influenced by the diagnostic criteria used.

As the concept $\mathrm{VCI}$ can also be used for describing patients at risk for becoming demented, we investigated in chapter VII whether the concept Mild Cognitive Impairment (MCI), specified in amnestic $\mathrm{MCI}$, multiple domains $\mathrm{MCI}$, and single non-memory domain $\mathrm{MCI}$, has prognostic validity in predicting $\mathrm{AD}$ or $\mathrm{VaD}$. Patients from a memory clinic $(\mathrm{N}=118)$ were compared to patients from a stroke unit $(\mathrm{N}=80)$. Patients from both cohorts were older than 55 years and with a cognitive deficit in at least one domain (without dementia). Multiple domains $\mathrm{MCI}^{\prime}$ had the highest sensitivity for both $\mathrm{AD}(80.8 \%)$ and $\mathrm{VaD}(100 \%)$, and 'amnestic $\mathrm{MCI}^{\prime}$ had the highest specificity (85.9\% for $\mathrm{AD}, 100 \%$ for $\mathrm{VaD}$ ). The positive predictive value was low for all subtypes $(0.0-32.7 \%)$, whereas the negative predictive value was high (72.8 - 100\%). We concluded that although the positive predictive value was low, the concept 'multiple domains impaired MCI' has high sensitivity in identifying patients at risk for dementia.

In chapter VIII reversible MCI (meaning permanent recovery) was investigated. One-hundred eighteen patients with a first-ever cerebral stroke and with MCI 
(defined as a cognitive deficit in at least one domain but not being demented) at baseline were followed up for 2 years. Possible predictors of reversible MCI were demographic variables, baseline MMSE-scores, presence of stroke risk factors, and CT-variables. Twenty-four (20.3\%) patients were classified as having permanent reversible MCI. Higher baseline-MMSE scores and female sex were independent predictors of recovery. We concluded that recovery from cognitive deficits is possible, and that this recovery is related to higher baseline MMSE score and female sex.

In chapter IX we evaluated whether stroke related and demographic variables are related to vascular cognitive impairment at 1, 6, and 12 months after stroke. Three groups of patients with dementia, vascular cognitive impairment (VCI), or vascular mild cognitive impairment (MCI) were compared with patients without cognitive disorders. At 1 month none of the variables were predictors of dementia; at 6 months low education $(\mathrm{OR}=13.2)$ and territorial infarct (OR $=16.2)$ predicted dementia; and at 12 months older age $(\mathrm{OR}=1.1)$ predicted dementia. Predictors of VCI were low education $(\mathrm{OR}=3.3)$ and territorial infarct $(\mathrm{OR}=2.4)$ at 1 month; older age $(\mathrm{OR}=1.1)$ and low education $(\mathrm{OR}=3.34)$ at 6 months; and older age $(\mathrm{OR}=1.1)$ at 12 months. Predictors of vascular MCI were low education $(\mathrm{OR}=4.9)$ and territorial infarct $(\mathrm{OR}=3.6)$ at 1 month; and older age and lower education at 6 months $(\mathrm{OR}=1.1$ and $\mathrm{OR}=3.9$ respectively) and at 12 months ( $\mathrm{OR}=1.1$ and $\mathrm{OR}=2.3$ respectively). Territorial infarct, older age, and low educational level, are predictors of cognitive disorders after stroke.

In chapter $X$ we tested the hypothesis that progressive brain damage is related to cognitive decline. In this chapter 101 patients (with both baseline and 2 years follow-up scan) were followed up for 2 years. Progression in white matter lesions and (silent) infarcts was recorded. Patients with progressive brain damage performed worse on cognitive tasks, both 1 and 24 months after stroke, yet changes in cognitive functioning was not different from that of patients without progressive brain damage. We concluded that there is not a simple relation between progression of ischemic brain damage and decline in cognitive functioning after first-ever stroke. Future research should address this issue in a larger stroke population.

In chapter XI we presented a new screening instrument (CLCE-24) and aspects of the feasibility and validity were investigated. The CLCE-24 was assessed in 69 stroke patients at 6 months after stroke. The CLCE- 24 was validated against the neuropsychological test data from CODAS. Patients and assessors are positive about its use. Eighty percent had cognitive and/or emotional problems; $73 \%$ had cognitive problems, while $51 \%$ had emotional problems. Correlations between the CLCE-24 and MMSE, CAMCOG, and SCL-90 were significant. The 
CLCE-24 is a significant predictor of the MMSE (Adj R2=0.31) and CAMCOG (Adj $\mathrm{R} 2=0.16$ ) at 12 months post stroke. The sensitivity is above $80 \%$, the specificity varies between 22 and $47 \%$. The CLCE- 24 is a feasible and valid instrument. Further research and implementation in clinical practice is recommended.

Whether psychiatric symptoms are risk factors for VCI was investigated in chapter XII. In this chapter patients with psychiatric symptoms (55.5\%) at baseline were compared to patients without psychiatric symptoms ( $54.5 \%$ ) in terms of risk for being diagnosed with either VaD or vascular MCI. Patients with psychiatric symptoms have a 7-fold chance of being diagnosed with $\mathrm{VaD}$ compared to patients without such complaints. Moreover, we found that patients with psychiatric complaints are at increased risk for cognitive deterioration. We concluded that only in very severe cognitive deficits psychiatric symptoms are related to cognitive deficits. Whether this is due to an underlying pathophysiological mechanism should be established in future research.

In chapter XIII we evaluated the results of the CODAS study. The aim of the CODAS study was to investigate the phenomenology of $\mathrm{VCI}$, its course and risk factors. It may be concluded that the cognitive profile of patients with VCI is characterised by mental speed problems and that although most patients remained stable in cognitive functioning, a substantial part of the patients improved. Further study is needed to investigate whether this cognitive profile is attributed to a specific underlying neuropathology. Older patients and patients with territorial infarcts are at risk for developing VCI. Moreover, psychiatric symptoms seem to be risk factors for post stroke cognitive deficits. Our findings contribute to a better knowledge of the concept VCI. However, they also imply that further research is needed especially in homogeneous subgroups, based on the underlying pathology. 
Samenvatting 
Beroerte, is na een hartinfarct en kanker doodsoorzaak nummer 1 in Westerse landen. Een beroerte gaat doorgaans gepaard met een hoge mate van blijvende handicap zowel op het fysieke vlak als mentaal. Recentelijk krijgen cognitieve stoornissen als gevolg van een beroerte meer aandacht. De meeste onderzoekers die de relatie tussen een beroerte en het cognitief functioneren bestuderen, richtten zich op het ontstaan van vasculaire dementie $(\mathrm{VaD})$. Echter, omdat het concept $\mathrm{VaD}$ de suggestie wekt dat het om één enkele ziektebeeld gaat met slechts één onderliggende pathologie, wordt het begrip steeds vaker in twijfel getrokken. Het breder concept Vascular Cognitive Impairment (VCI) omzeild deze problemen. In het concept VCI, staat 'vascular' voor alle oorzaken van ischemische vasculaire hersenschade en 'cognitive impairment' omvat alle vormen van cognitieve stoornissen. Ondanks dat steeds meer onderzoek over cognitieve gevolgen van een beroerte zich richt op VCI blijven een aantal zaken onopgehelderd. Zo is het onduidelijk hoe VCI zich uit, welke neuropsychologisch profiel past bij VCI en hoe het beloop van de cognitieve stoornissen is. Bovendien bestaat er nog onduidelijkheid over de risicofactoren die een rol spelen bij het ontstaan van VCI. Meer kennis is nodig om deze hiaten in de kennis in te vullen, voordat criteria voor VCI opgesteld kunnen worden om in de toekomst mensen te identificeren die at risk zijn voor het ontstaan van VCI.

In hoofdstuk II zijn we nader ingegaan op het concept VCI. Ondanks dat het concept VaD wijdverbreid wordt toegepast kent het een aantal problemen. Zo wordt de term 'vasculair', slecht omschreven in de diagnostische systemen. De meeste criteria operationaliseren onvoldoende de onderliggende pathologie. De diagnose 'dementie' in het concept $\mathrm{VaD}$ is gebaseerd op die van de ziekte van Alzheimer. Als gevolg hiervan wordt te veel waarde gehecht aan de aanwezigheid van een geheugenstoornis bij de diagnose $\mathrm{VaD}$. Het bredere concept VCI werd door Bowler en Hachinski geïntroduceerd, om het profiel van cognitieve stoornissen als gevolg van vasculaire hersenschade te beschrijven. VCI omvat niet enkel dementie, maar ook mildere cognitieve stoornissen.

In hoofdstuk III beschreven we de methodologische aspecten van het CODAS onderzoek. In het hoofdstuk gingen we dieper in op de methodologische keuzes die gemaakt zijn in CODAS, zoals het design, de procedure, de patiëntpopulatie, de neuropsychologische tests en medische gegevens en hoe we missing data hebben verwerkt. Bovendien bespreken we in dit hoofdstuk welke definities wij hebben gehanteerd om Mild Cognitive Impairment, VaD en VCI te identificeren en hoe we vooruitgang of achteruitgang in cognitief functioneren definiëren.

In hoofdstuk IV onderzochten we het geheugen, executief functioneren, mentale snelheid en het algeheel cognitief functioneren. Patiënten met een beroerte $(\mathrm{N}$ $=101$ ) werden vergeleken met een normpopulatie die gematched waren op 
leeftijd, opleiding en geslacht. We vonden dat de meeste cognitieve stoornissen in het gebied van mentale snelheid ( $50 \%$ van de patiënten) voorkwamen. De meeste patiënten presteerden op een later meetmoment hetzelfde als eerder (variërend van $37.6 \%$ tot $83.5 \%$ ), maar een groot deel van de patiënten herstelde (variërend van $12.9 \%$ tot $52.1 \% \%$ ). Oudere patiënten en vrouwelijke patiënten hadden vaker een cognitieve stoornis. Concluderend, kunnen we stellen dat de prognose gunstig is na een beroerte vooral voor jonge patiënten.

Cognitief functioneren op de lange termijn werd beschreven in hoofdstuk $V$. We onderzochten het voorkomen van dementie, $\mathrm{MCI}$ en van amnestic $\mathrm{MCI}$ op verschillende tijden na de beroerte. Bovendien werd het cognitief functioneren op verschillende neuropsychologische tests en het beloop van dit functioneren onderzocht. De prevalentie van VaD was op elk meetmoment $10 \%$. De meeste patiënten werden gediagnosticeerd als post-stroke MCI. Het cognitief functioneren verbeterde op bijna alle neuropsychologische tests 6 en 12 maanden na de beroerte. De frequentie van MCI na een beroerte hoog is, maar de frequentie van $\mathrm{VaD}$ is laag.

In hoofdstuk VI onderzochten we wat de invloed is van het gebruik van verschillende diagnostische criteria op de prevalentie en incidentie van VaD. Op 1, 6, 12 en 24 maanden na de beroerte werd uitgebreid klinisch onderzoek verricht bij 194 patiënten. VaD werd gediagnosticeerd volgens de DSM-III, DSM-III-R, DSM-IV, ICD-10-NA, NINDS-AIREN en de ADDTC criteria. De prevalentie van VAD was het hoogste direct na de beroerte en varieerde van $11.3 \%$ volgens de NINDS-AIREN tot $20.1 \%$ volgens de ICD-10-NA criteria. De incidentie was het hoogste 6 maanden na de beroerte, variërend van $2.6 \%$ volgens de ADDTC tot $5.2 \%$ volgens de ICD-10-NA. Wij concludeerde dat zowel de prevalentie als de incidentie wordt beïnvloed door het gebruik van verschillende criteria.

Omdat het concept VCI ook wordt toegepast om patiënten te identificeren die een verhoogd risico hebben op dementie onderzochten we in hoofdstuk VII of het concept Mild Cognitive Impairment (MCI) en meer specifiek, 'amnestic $\mathrm{MCI}^{\prime}$, 'meerdere domeinen $\mathrm{MCI}^{\prime}$ en 'een niet-geheugen gerelateerd domein $\mathrm{MCI}^{\prime}$, prognostische waarden heeft voor het ontwikkelen van Alzheimers dementie (AD) of VaD. Patiënten van een geheugenpoli $(\mathrm{N}=118)$ werden vergeleken met patiënten van een stroke unit $(\mathrm{N}=80)$. Patiënten van beide cohorten waren ouder dan 55 jaar en hadden ten minste op een cognitief gebied een stoornis, maar geen dementie. 'Meerder domeinen $\mathrm{MCI}^{\prime}$ had de hoogste sensitiviteit ( $80.8 \%$ voor $\mathrm{AD}$ en $100 \%$ voor $\mathrm{VaD}$ ), terwijl 'amnestic $\mathrm{MCI}^{\prime}$ de hoogste specificiteit had (85.9\% voor AD en $100 \%$ voor $\mathrm{VaD})$. De positief voorspellende waarde was laag voor alle $\mathrm{MCI}$ subtypes $(0.0-32.7 \%)$, terwijl de negatief voorspellende waarde hoog was $(72.8-100 \%)$. Wij concludeerden dat ondanks de lage positief 
voorspellende waarde, 'meerdere domeinen $\mathrm{MCI}^{\prime}$ hoge sensitiviteit heeft om patiënten te identificeren die een verhoogd risico hebben op dementie.

In hoofdstuk VIII werd onderzocht of herstel van MCI mogelijk is. Patiënten met een beroerte die op baseline gediagnosticeerd werden met MCI werden 2 jaar gevolgd. Mogelijke voorspellers voor herstel van MCI waren: demografische variabelen, baseline MMSE score, aanwezigheid van beroerte gerelateerde risicofactoren en CT-variabelen. Vierentwintig patiënten (20.3\%) herstelden van MCI. Hoger baseline MMSE score en vrouwelijk geslacht hebben een gunstige invloed op het beloop van MCI. We kunnen stellen dat na een beroerte herstel van MCI mogelijk is en dat dit herstel gerelateerd is aan hogere MMSE score en vrouwelijk geslacht.

In hoofdstuk IX beschreven we de relatie tussen demografische en beroerte gerelateerde variabelen en cognitieve stoornissen op 1, 6 en 12 maanden na het infarct. Drie patiëntgroepen ( $\mathrm{VaD}$, vascular $\mathrm{MCI}$ en $\mathrm{VCI})$ werden vergeleken met patiënten zonder cognitieve stoornissen. Op 1 maand na het infarct was geen enkele variabelen geassocieerd met $\mathrm{VaD}$; op 6 maanden na de beroerte waren dit lage opleiding $(\mathrm{OR}=13.2)$ en corticaal infarct $(\mathrm{OR}=16.2)$; op 12 voorspelde leeftijd $(\mathrm{OR}=1.1)$ dementie. Voorspellers van VCI waren lage opleiding $(\mathrm{OR}=3.3)$ en corticaal infarct $(\mathrm{OR}=2.4)$ op 1 maand; ouderdom $(\mathrm{OR}=1.1)$ en lage opleiding $(\mathrm{OR}=3.3)$ op 6 maanden; en ouderdom $(\mathrm{OR}=1.1)$ op 12 maanden na het infarct. Voorspellers voor vascular MCI waren lage opleiding $(\mathrm{OR}=4.9)$ en corticaal infarct $(\mathrm{OR}=3.6)$ op 1 maand, ouderdom en lager opleiding $(\mathrm{OR}=$ 1.1 en $\mathrm{OR}=3.9$ respectievelijk) op 6 maanden en ook op 12 maanden $(\mathrm{OR}=1.1$ en $\mathrm{OR}=2.3$ respectievelijk) na het infarct. Corticaal infarct, ouderdom en lage opleiding zijn voorspellers voor cognitieve stoornissen na een infarct.

In hoofdstuk X toetsten we de hypothese dat toenemende hersenschade in het brein gerelateerd is aan cognitieve achteruitgang. In dit onderzoek werden 101 patiënten (die zowel baseline als een 2-jaars follow-up CT scan hadden) gevolgd voor 2 jaar. Toename in witte stof beschadiging en in (stille) infarcering werd onderzocht. Patiënten met toegenomen hersenbeschadiging presteerden slechter (zowel op 1 als op 24 maanden) op de cognitieve taken dan de patiënten zonder deze toename, terwijl de veranderingen in cognitief functioneren vergelijkbaar waren tussen de twee groepen. Er is dus blijkbaar geen simpele een op een relatie tussen toegenomen hersenbescha-diging en achteruitgang in cognitief functioneren. Toekomstig onderzoek moet dit nadere bekijken in een groter CVA-populatie.

In hoofdstuk XI presenteerden we een nieuw signaleringinstrument (CLCE-24) en gingen we dieper in op de toepasbaarheid en de validiteit van dit instru- 
ment. De CLCE-24 werd afgenomen bij 69 patiënten 6 maanden na hun beroerte. De CLCE-24 werd afgezet tegen het cognitief functioneren gemeten met een neuropsychologisch onderzoek. Zowel de patiënt als de persoon die de test afnam waren positief over het instrument. Cognitieve klachten en emotionele problemen kwamen voor bij $80 \%$ van de mensen, $73 \%$ had cognitieve klachten en 51\% emotionele. Correlaties tussen de CLCE-24 en MSSE, CAMCOG en SCL90 waren positief en significant. De CLCE-24 had een voorspellende waarde voor het cognitief functioneren op 12 maanden na de beroerte (Adj OR $=0.31$ voor de MMSE en Adj $R=0.16$ voor de CAMCOG). De sensitiviteit was hoger dan $80 \%$ en de specificiteit varieerde van 22 tot $47 \%$. De CLCE- 24 is een handzaam en valide instrument om cognitieve en emotionele klachten na een beroerte op te sporen. Toekomstig onderzoek moet zich richten op de implementatie in de klinische praktijk.

In hoofdstuk XII onderzochten we of psychiatrische symptomen van invloed zijn op het cognitief functioneren na een beroerte. In dit hoofdstuk worden cvapatiënten met psychische symptomen $(55.5 \%)$ vergeleken met cva-patiënten zonder deze psychische symptomen (45,5\%) op het voorkomen van cognitieve stoornissen (VaD of vascular MCI). Patiënten met psychische symptomen hebben 7 keer zoveel kans om als $\mathrm{VaD}$ te worden gediagnosticeerd dan mensen zonder psychische symptomen. Bovendien hebben mensen met psychische symptomen een grotere kans om cognitief achteruit te gaan. We kunnen stellen dat de relatie tussen psychische symptomen en cognitie alleen terug te vinden is bij ernstige cognitieve stoornissen. Of deze relatie ten grondslag ligt aan een zelfde onderliggende pathologie moet in toekomstig onderzoek worden bekeken.

In hoofdstuk XIII beschreven we de bevindingen in dit proefschrift en hun betekenis. Het doel van de CODAS studie was om de fenomenologie, het beloop en de risicofactoren van VCI in kaart te brengen. We kunnen concluderen dat het cognitief profiel van patiënten met VCI wordt gekenmerkt door snelheidsproblemen en dat ondanks dat de meeste patiënten niet veranderen qua cognitief functioneren, herstelt een belangrijk deel. In toekomstig onderzoek moet onderzocht worden of het cognitieve profiel dat wij hebben gevonden past bij één specifieke onderliggende neuropathologische oorzaak. Oudere patiënten, patiënten met corticale infarcten en patiënten met psychische symptomen hebben een groter risico op VCI. Onze bevindingen dragen bij aan een beter begrip over het concept $\mathrm{VCI}$, maar ze zetten ook aan tot het opzetten van nieuw onderzoek naar VCI in homogene(gebaseerd op de onderliggende neuropathologische schade) subgroepen. 
References 
1. Lodder J, Bouter LM. [Future numbers of patients with a cerebrovascular accident in The Netherlands (see comments)]. Ned Tijdschr Geneeskd, 1992. 136(9):425-8.

2. Pohjasvaara T, Erkinjuntti T, Vataja R, Kaste M. Dementia three months after stroke. Baseline frequency and effect of different definitions of dementia in the Helsinki Stroke Aging Memory Study (SAM) cohort. Stroke, 1997. 28(4):785-92.

3. Pohjasvaara T, Mantyla R, Ylikoski R, et al. Comparison of different clinical criteria (DSM-III, DDTC, ICD-10, NINDS-AIREN, DSM-IV) for the diagnosis of vascular dementia. National Institute of Neurological Disorders and Stroke-Association Internationale pour la Recherche et l'Enseignement en Neurosciences. Stroke, 2000. 31(12):2952-7.

4. Verhey FRJ, Lodder J, Rozendaal N, Jolles J. Comparison of seven sets of criteria used for the diagnosis of vascular dementia. Neuroepidemiology, 1996. 15(3):166-72.

5. Wetterling T, Kanitz RD, Borgis KJ. Comparison of different diagnostic criteria for vascular dementia (ADDTC, DSM-IV, ICD-10, NINDSAIREN). Stroke, 1996. 27(1):30-6.

6. Tatemichi TK, Desmond DW, Mayeux R, et al. Dementia after stroke: baseline frequency, risks, and clinical features in a hospitalized cohort. Neurology, 1992. 42(6):1185-93.

7. Bowler JV, Hachinski V. Vascular Cognitive impairment: a new approach to vascular dementia, in Vascular Dementia. 1995. 357-376.

8. Ballard C, Stephens S, McLaren A, et al. Mild Cognitive Impairment and Vascular Cognitive Impairment in stroke patients. International Psychogeriatrics, 2003. 15(1):123-6.

9. de Jager CA, Hogervorst E, Combrinck M, Budge MM. Sensitivity and specificity of neuropsychological tests for mild cognitive impairment, vascular cognitive impairment and Alzheimer's disease. Psychol Med, 2003. 33(6):1039-50.

10. Ingles JL, Wentzel C, Fisk JD, Rockwood K. Neuropsychological predictors of incident dementia in patients with vascular cognitive impairment, without dementia. Stroke, 2002. 33(8):1999-2002.

11. Rockwood K, Ebly E, Hachinski V, Hogan D. Presence and treatment of vascular risk factors in patients with vascular cognitive impairment. Arch Neurol, 1997. 54(1):33-9.

12. Rockwood K, Wentzel C, Hachinski V, et al. Prevalence and outcomes of vascular cognitive impairment. Vascular Cognitive Impairment Investigators of the Canadian Study of Health and Aging. Neurology, 2000. 54(2):447-51. 
13. Szatmari S, Fekete I, Csiba L, et al. Screening of vascular cognitive impairment on a Hungarian cohort. Psychiatry Clin Neurosci, 1999. 53(1):39-43.

14. Wentzel C, Darvesh S, MacKnight C, et al. Inter-rater reliability of the diagnosis of vascular cognitive impairment at a memory clinic. Neuroepidemiology, 2000. 19(4):186-93.

15. Wentzel C, Rockwood K, MacKnight C, et al. Progression of impairment in patientswith vascular cognitive impairment without dementia. Neurology, 2001. 57(4):714-6.

16. Censori B, Manara O, Agostinis C, et al. Dementia after first stroke. Stroke, 1996. 27(7):1205-10.

17. Desmond DW, Moroney JT, Paik MC, et al. Frequency and clinical determinants of dementia after ischemic stroke [In Process Citation]. Neurology, 2000. 54(5):1124-31.

18. Madureira S, Guerreiro M, Ferro JM. Dementia and cognitive impairment three months after stroke. Eur J Neurol, 2001. 8(6):621-7.

19. Whitehouse PJ, Lerner A, Hedera P. Dementia, in Clinical neuropsychology, K.M. Heilman and E. Valenstein, Editors. 1993, Oxford University Press: New York. 603-646.

20. Hachinski VC, Lassen NA, Marshall J. Multi-infarct dementia. A cause of mental deterioration in the elderly. Lancet, 1974. 2(7874):207-10.

21. Verhey FRJ. Vasculaire Dementie. Tijdschrift voor Psychiatrie, 1995. 37(3):180-193.

22. World Health Organisation (WHO). The neurological adaptation of the international classification of diseases (ICD-10). 1991, Geneva: WHO.

23. American Psychiatric Association: Diagnostic and Statistical Manual of Mental Disorders. 4 ed, ed. A.P. Association. 1994, Washington.

24. Roman GC, Tatemichi TK, Erkinjuntti T, et al. Vascular dementia: diagnostic criteria for research studies. Report of the NINDS-AIREN International Workshop [see comments]. Neurology, 1993. 43(2):250-60.

25. Chui HC, Victoroff JI, Margolin D, et al. Criteria for the diagnosis of ischemic vascular dementia proposed by the State of California Alzheimer's Disease Diagnostic and Treatment Centres. Neurology, 1992. 42(3 Pt 1):473-80.

26. Erkinjuntti T, Inzitari D, Pantoni L, et al. Research criteria for subcortical vascular dementia in clinical trials. J Neural Transm Suppl, 2000. 59: 23-30. 
27. Erkinjuntti T, Inzitari D, Pantoni L, et al. Limitations of clinical criteria for the diagnosis of vascular dementia in clinical trials. Is a focus on subcortical vascular dementia a solution? Ann N Y Acad Sci, 2000. 903: 262-72.

28. Rockwood K, Bowler J, Erkinjuntti T, et al. Subtypes of vascular dementia. Alzheimer Dis Assoc Disord, 1999. 13 Suppl 3:S59-65.

29. Rasquin SMC, Verhey FRJ, Lousberg R, et al. Vascular cognitive disorders. Memory, mental speed and cognitive flexibility after stroke. J Neurol Sci, 2002. 203-204(C):115-9.

30. Stewart R. Vascular dementia: a diagnosis running out of time. Br J Psychiatry, 2002. 180:152-6.

31. Pathological correlates of late-onset dementia in a multicentre, community-based population in England and Wales. Neuropathology Group of the Medical Research Council Cognitive Function and Ageing Study (MRC CFAS). Lancet, 2001. 357(9251):169-75.

32. Tatemichi TK, Desmond DW, Stern Y, et al. Cognitive impairment after stroke: frequency, patterns, and relationship to functional abilities. J Neurol Neurosurg Psychiatry, 1994. 57(2):202-7.

33. Meyer J, Xu G, Thornby J, et al. Longitudinal analysis of abnormal domains comprising mild cognitive impairment (MCI) during aging. J Neurol Sci, 2002. 201(1-2):19.

34. Cote R, Battista RN, Wolfson C, et al. The Canadian Neurological Scale: validation andreliability assessment. Neurology, 1989. 39(5):638-43.

35. Rankin J. Cerebral vascular accidents in patients over the age of 60. III. Diagnosis and treatment. Scott Med J, 1957. 2(6):254-68.

36. De Graaf A, Deelman BG. De Cognitieve Screening Test. 1992, Lisse: Swets en Zeitlinger.

37. Derogatis LR, Lipman RS, Covi L. SCL-90: an outpatient psychiatric rating scale-preliminary report. Psychopharmacol Bull, 1973. 9(1):13-28.

38. Teunisse S, Derix MMA. [Measurement of activities of daily living in patients with dementia living at home: development of a questionnaire (see comments)]. Tijdschr Gerontol Geriatr, 1991. 22(2):53-9.

39. Verhey FRJ, Huppert FA, Korten ECCM, et al. Cross-sectional comparison of the Cambridge Cognitive Examination -revised: The CAMCOG-R. Age and Aging, 2003. 32:1-7.

40. de Koning I, van Kooten F, Dippel DW, et al. The CAMCOG: a useful screening instrument for dementia in stroke patients. Stroke, 1998. 29(10):2080-6. 
41. Folstein MF, Folstein SE, McHugh PR. “Mini-mental state”. A practical method for grading the cognitive state of patients for the clinician. J Psychiatr Res, 1975. 12(3):189-98.

42. Brand PA, Jolles J. Learning and retrieval rate of words presented auditorialy and visually. The Journal of General Psychology, 1985. 112:201210.

43. Reitan RM. Validity of the Trail Making Test as an indication of organic brain damage. Perceptual and motor skills, 1985. 8:271-276.

44. Houx PJ. Cognitive aging and health related factors. Doctoral Thesis University of Maastricht, 1991.

45. Houx PJ, Jolles J, Vreeling FW. Stroop interference: aging effects assessed with the Stroop Color-Word Test. Experimental Aging Research, 1993. 19(204-224).

46. Bouma A, Mulder T, Lindeboom J. Neuropsychologische diagnostiek. Handboek. 1996, Lisse: Swets \& Zeitlinger Publishers.

47. Luteijn F, van der Ploeg FAE. [Manual Groninger Intelligence Test]. 1983, Lisse, The Netherlands: Swets and Zeitlinger.

48. Rozenboom WW. The reliability of a linear composite of nonequivalent subtests. Applied Psychological Measurement, 1989. 13:277-283.

49. Jolles J, Houx PJ, van Boxtel MPJ, et al. The Maastricht Aging Study: Determinants of cognitive aging. 1995, Maastricht: Neuropsych Publishers.

50. Derix MMA, Hofstede AB, Teunisse S, et al. [CAMDEX-N: the Dutch version of the Cambridge Examination for Mental Disorders of the Elderly with automatic data processing]. Tijdschr Gerontol Geriatr, 1991. 22(4):143-50.

51. Tabachnick BG, Fidell LS. Using multivariate statistics. 2001, Boston: Allyn \& Bacon.

52. Boiten J, Lodder J. Lacunar Infarcts. Pathogenesis and validity of the clinical syndromes. Stroke, 1991. 22(11):1374-8.

53. de Jong G, van Raak L, Kessels F, Lodder J. Stroke subtype and mortality. a follow-up study in 998 patients with a first cerebral infarct. J Clin Epidemiol, 2003. 56(3):262-8.

54. van Zagten M, Boiten J, Kessels F, Lodder J. Significant progression of white matter lesions and small deep (lacunar) infarcts in patients with stroke. Arch Neurol, 1996. 53(7):650-5.

55. Leys D, Pruvo JP, Petit H, et al. [Maladie d'Alzheimer: anlyse statistique de resultats du scanner X]. Rev Neurol (Paris), 1989. 145:134-139.

56. Bowler JV, Hadar U, Wade JP. Cognition in stroke. Acta Neurol Scand, 1994. 90(6):424-9. 
57. Wade DT, Skilbeck C, Hewer RL. Selected cognitive losses after stroke. Frequency, recovery and prognostic importance. Int Disabil Stud, 1989. 11(1):34-9.

58. Ebrahim S, Nouri F, Barer D. Cognitive impairment after stroke. Age Ageing, 1985. 14(6):345-8.

59. Kase CS, Wolf PA,Kelly-Hayes M, et al. Intellectual decline after stroke: the Framingham Study. Stroke, 1998. 29(4):805-12.

60. Wilson LA, Brass W. Brief assessment of mental state in geriatric domiciliary practice: the usefulness of the mental state questionnaire. Age Ageing, 1973. 2:92-101.

61. Prevo AJ, Dijkman MM, Le Fevre FA. [Impairment and disability in patients with a severe ischemic cerebral infarction at admission to the rehabilitation center and six months after stroke]. Ned Tijdschr Geneeskd, 1998. 142(12):637-40.

62. Barba R, Martinez-Espinosa S, Rodriguez-Garcia E, et al. Poststroke dementia : clinical features and risk factors. Stroke, 2000. 31(7):1494-501.

63. Chui H. Dementia due to subcortical ischemic vascular disease. Clin Cornerstone, 2001. 3(4):40-51.

64. Erkinjuntti T. Vascular Dementia Current Concepts Clinicopathological Study of Vascular Dementia. 1st ed. 1996, Chichester: John Wiley \& Sons Ltd. 73-112.

65. Kwa VI, Limburg M, Voogel AJ, et al. Feasibility of cognitive screening of patients with ischaemic stroke using the CAMCOG. A hospital-based study. J Neurol, 1996. 243(5):405-9.

66. Roth M. The Diagnosis of Dementia in Late and Middle Life, in The Epidemiology of Dementia. 1981, Oxford University Press: New York Oxford. 24-61.

67. Almkvist O. Neuropsychological deficits in vascular dementia in relation to Alzheimer's disease: reviewing evidence for functional similarity or divergence. Dementia, 1994. 5 (34):203-9.

68. Boone KB, Miller BL, Lesser IM, et al. Neuropsychological correlates of white-matter lesions in healthy elderly subjects. A threshold effect. Arch Neurol, 1992. 49(5):549-54.

69. de Groot JC, de Leeuw FE, Oudkerk M, et al. Cerebral white matter lesions and cognitive function: the Rotterdam Scan Study [see comments]. Ann Neurol, 2000. 47(2):145-51.

70. van Gijn J. Leukoaraiosis and vascular dementia. Neurology, 1998. 51 (Suppl 3):S3-8. 
71. Desmond DW, Tatemichi TK, Paik M, Stern Y. Risk factors for cerebrovascular disease as correlates of cognitive function in a stroke-free cohort. Arch Neurol, 1993. 50(2):162-6.

72. Zhu L, Fratiglioni L, Guo Z, et al. Association of stroke with dementia, cognitive impairment, and functional disability in the very old: a population-based study. Stroke, 1998. 29(10):2094-9.

73. Hochstenbach J, Mulder T, Limbeek J. [The neuropsychology of stroke: changes in cognition, emotion and behaviour]. TSG, 1997. 75:479-485.

74. Leys D, Henon H, Pasquier F. White matter changes and poststroke dementia. Dement Geriatr Cogn Disord, 1998. 9 Suppl 1:25-9.

75. Tatemichi TK, Desmond DW, Prohovnik I, et al. Confusion and memory loss from capsular genu infarction: a thalamocortical disconnection syndrome? [see comments]. Neurology, 1992. 42(10):1966-79.

76. Rasquin SMC, Verhey FRJ, Lodder J. [Vascular Cognitive Impairment: a new concept. Nederlands Tijdschrift voor Neurologie, 2003. 104(4):209214.

77. Ballard C, Stephens S, Kenny R, et al. Profile of Neuropsychological Deficits in Older Stroke Survivors without Dementia. Dement Geriatr Cogn Disord, 2003. 16(1):52-6.

78. Frisoni GB, Galluzzi S, Bresciani L, et al. Mild cognitive impairment with subcortical vascular features. Clinical characteristics and outcome. J Neurol, 2002. 249(10):1423-32.

79. Ebly EM, Hogan DB, Parhad IM. Cognitive impairment in the nondemented elderly. Results from the Canadian Study of Health and Aging. Arch Neurol, 1995. 52(6):612-9.

80. Petersen RC, Smith GE, Waring SC, et al. Mild cognitive impairment: clinical characterization and outcome. Arch Neurol, 1999. 56(3):303-8.

81. Petersen RC, Doody R, Kurz A, et al. Current concepts in mild cognitive impairment. Arch Neurol, 2001. 58(12):1985-92.

82. Vink M, Jolles J. A new version of the Trail-Making Test as an information processing task. Journal of Clinical Neuropsychology, 1985. 7:162.

83. van Boxtel MP, Langerak K, Houx PJ, Jolles J. Self-reported physical activity, subjective health, and cognitive performance in older adults. Exp Aging Res, 1996. 22(4):363-79.

84. Jacobson NS, Truax P. Clinical significance: a statistical approach to defining meaningful change in psychotherapy research. J Consult Clin Psychol, 1991. 59(1):12-9.

85. Hafkenscheid A. [The incorrigible tendency of 'improvement' of the change-scores: back to the original index for reliable changes]. Gedragstherapie, 2002. 35(4):317-327. 
86. Henon H, Durieu I, Guerouaou D, et al. Poststroke dementia: incidence and relationship to prestroke cognitive decline. Neurology, 2001. 57(7): 1216-22.

87. Henon H, Pasquier F, Durieu I, et al. Preexisting dementia in stroke patients. Baseline frequency, associated factors, and outcome. Stroke, 1997. 28(12):2429-36.

88. Leskela M, Hietanen M, Kalska H, et al. Executive functions and speed of mental processing in elderly patients with frontal or nonfrontal ischemic stroke. Eur J Neurol, 1999. 6(6):653-61.

89. Desmond DW, Moroney JT, Sano M, Stern Y. Recovery of cognitive function after stroke. Stroke, 1996. 27(10):1798-803.

90. Schmidt R, Mechtler L, Kinkel PR, et al. Cognitive impairment after acute supratentorial stroke: a 6-month follow-up clinical and computed tomographic study. Eur Arch Psychiatry Clin Neurosci, 1993. 243(1):115 .

91. Ballard C, Rowan E, Stephens S, et al. Prospective follow-up study between 3 and 15 months after stroke: improvements and decline in cognitive function among dementia-free stroke survivors $>75$ years of age. Stroke, 2003. 34(10):2440-4.

92. Vermeer SE, Prins ND, den Heijer T, et al. Silent brain infarcts and the risk of dementia and cognitive decline. N Engl J Med, 2003. 348(13):121522.

93. Lezak MD. Neuropsychological Assessment. third ed. 1995, New York: Oxford University Press.

94. American Psychiatric Association: Diagnostic and Statistical Manual of Mental Disorders. 3 ed. American Psychiatric Association. 1980, Washington.

95. American Psychiatric Association: Diagnostic and Statistical Manual of Mental Disorders. 3 revised ed. 1987, Washington.

96. World Health Organization (WHO). The Neurological Adaptation of the International Classification of Disease (ICD-10NA). 1991, Geneva: World Health Organization.

97. Hebert R, Brayne C. Epidemiology of vascular dementia. Neuroepidemiology, 1995. 14(5):240-57.

98. Tatemichi TK, Foulkes MA, Mohr JP, et al. Dementia in stroke survivors in the Stroke Data Bank cohort. Prevalence, incidence, risk factors, and computed tomographic findings. Stroke, 1990. 21(6):858-66.

99. Treves TA, Aronovich BD, Bornstein NM, Korczyn C. Risk of Dementia after a Firts-Ever Ischemic Stroke: A 3-Year Longitudinal Study.

Cerebrovasc Dis, 1997. 7:48-52. 
100. Rasquin SMC, Verhey FRJ, van Oostenbrugge RJ, et al. Demographic and CT-scan features related to cognitive impairment in the first year after stroke. Journal of Neurology, Neurosurgery, and Psychiatry, 2004. in press.

101. Rasquin SMC, Lodder J, Ponds RWMH, et al. Cognitive functioning after stroke: a one year follow-up study. Dem and Geriatric Cogn. Disorders, 2004. 18:138-144.

102. Landis JR, Koch GG. The measurement of observer agreement for categorical data. Biomedics, 1977. 33:159-174

103. Gold G, Giannakopoulos P, Montes-Paixao C Jr., et al. Sensitivity and specificity of newly proposed clinical criteria for possible vascular dementia. Neurology, 1997. 49(3):690-4.

104. Amar K, Wilcock GK, Scott M. The diagnosis of vascular dementia in the light of the new criteria [see comments]. Age Ageing, 1996. 25(1):51-5.

105. Desmond DW, Moroney JT, Sano M, stern Y. Incidence of dementia after ischemic stroke: results of a longitudinal study. Stroke, 2002. 33(9): 2254-62.

106. Hachinski V. Preventable senility: a call for action against the vascular dementias [see comments]. Lancet, 1992. 340(8820):645-8.

107. Chui HC, Mack W, Jackson JE, et al. Clinical criteria for the diagnosis of vascular dementia: a multicenter study of comparability and interrater reliability [see comments]. Arch Neurol, 2000. 57(2):191-6.

108. Busse A, Bischkopf J, Riedel-Heller SG, Angmeyer MC.

Subclassifications for mild cognitive impairment: prevalence and predictive validity. Psychol Med, 2003. 33(6):1029-38.

109. DeCarli C. Mild Cognitive Impairment: prevalence, prognosis, aetiology, and treatment. Lancet, 2003. 2:15-21.

110. Ritchie K, Touchon. J Mild cognitive impairment: conceptual basis and current nosological status. Lancet, 2000. 355(9199):225-8.

111. Visser PJ, Verhey FRJ, Ponds RWHM, et al. Distinction between preclinical Alzheimer's disease and depression. J Am Geriatr Soc, 2000. 48(5): 479-84.

112. Tian J, Bucks RS, Haworth J, Wilcock G. Neuropsychological prediction of conversion to dementia from questionable dementia: statistically significant but not yet clinically useful. Neurol Neurosurg Psychiatry, 2003. 74(4):433-8.

113. Tham W, Auchus AP, Thong M, et al. Progression of cognitive impairment after stroke. One year results from a longitudinal study of Singaporean stroke patients. J Neurol Sci, 2002. 203-204(C):49-52. 
114. Fisk JD, Merry HR, Rockwood K. Variations in case definition affect prevalence but not outcomes of mild cognitive impairment. Neurology, 2003. 61(9):1179-84.

115. Shah Y, Tangalos EG, Petersen RC. Mild cognitive impairment. When is it a precursor to Alzheimer's disease? Geriatrics, 2000. 55(9):62, 65-8.

116. Ingles JL, Fisk JD, Merry HR, Rockwood K. Five-year outcomes for dementia defined solely by neuropsychological test performance. Neuroepidemiology, 2003. 22(3):172-8.

117. Larrieu S, Letenneur L, Orgogozo JM, et al. Incidence and outcome of mild cognitive impairment in a population-based prospective cohort. Neurology, 2002. 59(10):1594-9.

118. Palmer K, Fratiglioni L, Winblad B. What is mild cognitive impairment? Variations in definitions and evolution of nondemented persons with cognitive impairment. Acta Neurol Scand Suppl, 2003. 179:14-20.

119. Palmer K, Wang HX, Backman L, et al. Differential evolution of cognitive impairment in nondemented older persons: results from the Kungsholmen Project. Am J Psychiatry, 2002. 159(3):436-42.

120. Wolf H, Grunwald M, Ecke GM, et al. The prognosis of mild cognitive impairment in the elderly. J Neural Transm Suppl, 1998. 54:31-50.

121. Christensen H, Henderson AS, Korten AE, et al. ICD-10 mild cognitive disorder: its outcome three years later. Int J Geriatr Psychiatry, 1997. 12(5):581-6.

122. Wahlund LO, Pihlstrand E, Jonhagen ME. Mild cognitive impairment: experience from a memory clinic. Acta Neurol Scand Suppl, 2003. 179: 21-4.

123. Hochstenbach JB, den Otter R, Mulder TW. Cognitive recovery after stroke: a 2-year follow-up. Arch Phys Med Rehabil, 2003. 84(10):1499504.

124. Kotila M, Waltimo O, Niemi ML, et al. The profile of recovery from stroke and factors influencing outcome. Stroke, 1984. 15(6):1039-44.

125. Patel M, Coshall C, Rudd AG, Wolfe CD. Natural history of cognitive impairment after stroke and factors associated with its recovery. Clin Rehabil, 2003. 17(2):158-66.

126. Visser PJ, Verhey FRJ, Scheltens P, et al. Diagnostic accuracy of the Preclinical AD Scale (PAS) in cognitively mildly impaired subjects. J Neurol, 2002. 249(3):312-9.

127. Pohjasvaara T, Erkinjuntti T, Ylikoski R, et al. Clinical determinants of poststroke dementia. Stroke, 1998. 29(1):75-81.

128. Skoog I. Risk factors for vascular dementia: a review. Dementia, 1994. 5(3-4):137-44. 
129. House A, Dennis M, Warlow C, et al. The relationship between intellectual impairment and mood disorder in the first year after stroke. Psychol Med, 1990. 20(4):805-14.

130. Charletta D, Gorelick PB, and Dollear TJ, et al. CT and MRI findings among African Americans with Alzheimer's disease, vascular dementia, and stroke without dementia. Neurology, 1995. 45(8):1456-61.

131. Lin RT, Lai CL, Tai CT, et al. Cranial computed tomography in ischemic stroke patients with and without dementia--a prospective study. Kaohsiung J Med Sci, 1998. 14(4):203-11.

132. Loeb C, Gandolfo C, Bino G. Intellectual impairment and cerebral lesions in multiple cerebral infarcts. A clinical-computed tomography study. Stroke, 1988. 19(5):560-5.

133. Pohjasvaara T, Mantyla R, Salonen O, et al. How complex interactions of ischemic brain infarcts, white matter lesions, and atrophy relate to poststroke dementia. Arch of Neurol, 2000. 57:1295-1300.

134. Pohjasvaara T, Mantyla R, Salonen O, et al. MRI correlates of dementia after first clinical ischemic stroke. J Neurol Sci, 2000. 181(1-2):111-7.

135. Tatemichi TK, Desmond DW, and Paik M, et al. Clinical determinants of dementia related to stroke. Ann Neurol, 1993. 33(6):568-75.

136. Wentzel C, et al. Progression of impairment in patients with vascular cognitive impairment without dementia. Neurology, 2001. 57(4):714-6.

137. O'Brien JT, Erkinjuntti T, Reisberg B, et al. Vascular Cognitive Impairment. Lancet, 2003. 2:89-98.

138. Elwan O, Hashem S, Helmy AA, Wolfe CD. Cognitive deficits in ischemic strokes: psychometric, electrophysiological and cranial tomographic assessment. J Neurol Sci, 1994. 125(2):168-74.

139. Patel MD, Coshall C, Rudd AG, Wolfe CD. Cognitive impairment after stroke: clinical determinants and its associations with long-term stroke outcomes. J Am Geriatr Soc, 2002. 50(4):700-6.

140. Tatemichi TK, Desmond DW, and Paik M, et al. Clinical determinants of dementia related to stroke. Ann Neurol, 1993. 33(6):568-75.

141. Liu CK, Miller BL, Cummings JL, et al. A quantitative MRI study of vascular dementia. Neurology, 1992. 42(1):138-43.

142. Pennese F, Del Re ML, Esposito F. Clinical and tomographic findings in vascular dementia. Aging (Milano), 1994. 6(1):57-64.

143. Pohjasvaara T, Mantyla R, Salonen O, et al. How complex interactions of ischemic brain infarcts, white matter lesions, and atrophy relate to poststroke dementia. Arch Neurol, 2000. 57(9):1295-300. 
144. Henon H, Pasquier F, Durieu I, et al. Medial temporal lobe atrophy in stroke patients: relation to pre- existing dementia. J Neurol Neurosurg Psychiatry, 1998. 65(5):641-7.

145. Pasquier F, Leys D. Why are stroke patients prone to develop dementia? J Neurol, 1997. 244(3):135-42.

146. Pasquier F, Henon H, Leys D. Relevance of white matter changes to preand poststroke dementia. Ann N Y Acad Sci, 2000. 903:466-9.

147. Bernick C, Kuller L, and Dulberg C, et al. Silent MRI infarcts and the risk of future stroke: the cardiovascular health study. Neurology, 2001. 57(7): 1222-9.

148. Meyer JS, Obara K, Muramatsu K, et al. Cognitive performance after small strokes correlates with ischemia, not atrophy of the brain.

Dementia, 1995. 6(6):312-22.

149. Wardlaw JM, West TM, Sandercock PA, et al. Visible infarction on computed tomography is an independent predictor of poor functional outcome after stroke, and not of haemorrhagic transformation. J Neurol Neurosurg Psychiatry, 2003. 74(4):452-8.

150. Englund E, Brun A, Alling C. White matter changes in dementia of Alzheimer's type. Biochemical and neuropathological correlates. Brain, 1988. 111 ( Pt 6):1425-39.

151. Leys D, Englund E, Del Ser T, et al. White matter changes in stroke patients. Relationship with stroke subtype and outcome. Eur Neurol, 1999. 42(2):67-75.

152. Pantoni L, Leys D, Fazekas F, et al. Role of white matter lesions in cognitive impairment of vascular origin. Alzheimer Dis Assoc Disord, 1999. 13 Suppl 3:S49-54.

153. Bowler JV. Editorial comment--The progression of leukoaraiosis. Stroke, 2003. 34(8):19167.

154. Norrving B. Long-term prognosis after lacunar infarction. Lancet Neurol, 2003. 2(4):23845.

155. Roman GC. Vascular dementia revisited: diagnosis, pathogenesis, treatment, and prevention. Med Clin North Am, 2002. 86(3):477-99.

156. Meyer JS, Muramatsu K, Mortel KF, et al. Prospective CT confirms differences between vascular and Alzheimer's dementia. Stroke, 1995. 26(5):735-42.

157. Hachinski V. Vascular behavioral and cognitive disorders. Stroke, 2003. 34(12):2775.

158. Schmidt R, Schmidt H, Kapeller P, et al. The natural course of MRI white matter hyperintensities. J Neurol Sci, 2002. 203-204:253-7. 
159. Ballard C, O'Brien J, Morris CM, et al. The progression of cognitive impairment in dementia with Lewy bodies, vascular dementia and Alzheimer's disease. Int J Geriatr Psychiatry, 2001. 16(5):499-503.

160. Paul RH, Cohen RA, Moser DJ, et al. Clinical correlates of cognitive decline in vascular dementia. Cognitive and Behavioral Neurology, 2003. 16(1):40-46.

161. Hochstenbach J, Mulder T, van Limbeek J, et al. Cognitive decline following stroke: a comprehensive study of cognitive decline following stroke. J Clin Exp Neuropsychol, 1998. 20(4):503-17.

162. Aben I, Verhey FRJ, Strik J, et al. A comparative study into the one year cumulative incidence of depression after stroke and myocardial infarction. J Neurol Neurosurg Psychiatry, 2003. 74(5):581-5.

163. Sundet K, Finset A, Reinvang I. Neuropsychological predictors in stroke rehabilitation. J Clin Exp Neuropsychol, 1988. 10(4):363-79.

164. Kotila M, Niemi ML, Laaksonen R. Four-year prognosis of stroke patients with visuospatial inattention. Scand J Rehabil Med, 1986. 18(4): 177-9.

165. Sodring KM, Laake K, Sveen U, et al. Validation of the screening instrument for neuropsychological impairment in stroke. Physiother Res Int, 1998. 3(1):15-26.

166. van Zandvoort MJE. Cognitive function following single lacunar infarct. Doctoral Thesis. University of Utrecht, 2001.

167. Arrindell WA, Ettema JHM. Symptom Checklist [Handleiding bij een multidimensionele psychopathologie-indicator]. 2003, Lisse: Swets \& Zeitlinger B.V.

168. Visser PJ. Predictors of Alzheimers type dementia in subjects with mild cognitive impairments. Doctoral Thesis University Maastricht, 2000.

169. Forster A, Smith J, Young J, et al. Information provision for stroke patients and their caregivers. Cochrane database review 3 CD001919.

170. Hanger HC, Mulley GP. Questions people ask about stroke. Stroke, 1993. 24(4):536-8.

171. Visser-Keizer AC, Meyboom-de Jong B, Deelman BG, et al. Subjective changes in emotion, cognition and behaviour after stroke: factors affecting the perception of patients and partners. J Clin Exp Neuropsychol, 2002. 24(8):1032-45.

172. O'Connor DW, Pollitt PA, Brook CP, et al. Does early intervention reduce the number of elderly people with dementia admitted to institutions for long term care? Bmj, 1991. 302(6781):871-5.

173. Chemerinski E, Robinson RG. The neuropsychiatry of stroke. Psychosomatics, 2000. 41(1):5-14. 
174. Robinson RG. Neuropsychiatric consequences of stroke. Annu Rev Med, 1997. 48:21729.

175. Johnson GA. Research into psychiatric disorder after stroke: the need for further studies. Aust N Z J Psychiatry, 1991. 25(3):358-70.

176. Aben I, Verhey FRJ, Honig A, et al. Research into the specificity of depression after stroke: a review on an unresolved issue. Prog Neuropsychopharmacol Biol Psychiatry, 2001. 25(4):671-89.

177. Downhill JE Jr., Robinson RG. Longitudinal assessment of depression and cognitive impairment following stroke. J Nerv Ment Dis, 1994. 182(8):425-31.

178. Murata Y, Kimura M, Robinson RG. Does cognitive impairment cause post-stroke depression? Am J Geriatr Psychiatry, 2000. 8(4):310-7.

179. Robinson RG, Bolla-Wilson K, Kaplan E, et al. Depression influences intellectual impairment in stroke patients. Br J Psychiatry, 1986. 148:5417.

180. Shimoda K, Robinson RG. Effects of anxiety disorder on impairment and recovery from stroke. J Neuropsychiatry Clin Neurosci, 1998. 10(1):34-40.

181. Bowler JV. The concept of vascular cognitive impairment. J Neurol Sci, 2002. 203204(C):11-5.

182. Grant MM, Thase ME, Sweeney JA. Cognitive disturbance in outpatient depressed younger adults: evidence of modest impairment. Biol Psychiatry, 2001. 50(1):35-43.

183. Purcell R, et al. Neuropsychological function in young patients with unipolar major depression. Psychol Med, 1997. 27(6):1277-85.

184. Wilson RS, Mendes De Leon CF, Bennett DA, et al. Depressive symptoms and cognitive decline in a community population of older persons. J Neurol Neurosurg Psychiatry, 2004. 75(1):126-9.

185. Gerritsen MJ, Berg LJ, Deelman BG, et al. Speed of information processing after unilateral stroke. J Clin Exp Neuropsychol, 2003. 25(1):1-13.

186. Aben I, Verhey FRJ, Lousberg R, et al. Validity of the beck depression inventory, hospital anxiety and depression scale, SCL-90, and hamilton depression rating scale as screening instruments for depression in stroke patients. Psychosomatics, 2002. 43(5):386-93.

187. Robinson RG. Poststroke depression: prevalence, diagnosis, treatment, and disease progression. Biol Psychiatry, 2003. 54(3):376-87.

188. Carson AJ, MacHale S, Allen K, et al. Depression after stroke and lesion location: a systematic review. Lancet, 2000. 356(9224):122-6.

189. Castillo CS, Starkstein SE, Fedoroff JP, et al. Generalized anxiety disorder after stroke. J Nerv Ment Dis, 1993. 181(2):100-6. 
190. Desmond DW, Moroney JT, Bagiella E, et al. Dementia as a predictor of adverse outcomes following stroke: an evaluation of diagnostic methods. Stroke, 1998. 29(1):69-74.

191. Desmond DW, Moroney JT, Sano M, Stern Y. Mortality in patients with dementia after ischemic stroke. Neurology, 2002. 59(4):537-43.

192. Hajek VE, Gagnon S, Ruderman JE. Cognitive and functional assessments of stroke patients: an analysis of their relation. Arch Phys Med Rehabil, 1997. 78(12):1331-7.

193. Gauthier S, Rockwood K, Gelinas I, et al. Outcome measures for the study of activities of daily living in vascular dementia. Alzheimer Dis Assoc Disord, 1999. 13 Suppl 3:S143-7.

194. Giaquinto S, Buzzelli S, Di Francesco L, et al. On the prognosis of outcome after stroke. Acta Neurol Scand, 1999. 100(3):202-8.

195. Jonkman EJ, de Weerd AW, Vrijens NL. Quality of life after a first ischemic stroke. Long term developments and correlations with changes in neurological deficit, mood an cognitive impairment. Acta Neurol Scand, 1998. 98(3):169-75.

196. Gordon WA, Hibbard MR. Poststroke depression: an examination of the literature. Arch Phys Med Rehabil, 1997. 78(6):658-63.

197. Pohjasvaara T, Vataja R, Leppavuori A, et al. Depression is an independent predictor of poor long-term functional outcome post-stroke. Eur J Neurol, 2001. 8(4):315-9.

198. Pohjasvaara T, Vataja R, Leppavuori A, et al. Cognitive functions and depression as predictors of poor outcome 15 months after stroke. Cerebrovasc Dis, 2002. 14(3-4):228-33.

199. Almkvist O. Cognitive syndrome(s) in preclinical and clinical Vascular Dementia. International Psychogeriatrics, 2003. 15(1):127-31.

200. Ballard C, Patel A, Oyebode F, Wilcock C. Cognitive decline in patients with Alzheimer's disease, vascular dementia and senile dementia of Lewy body type. Age Ageing, 1996. 25(3):209-13.

201. Boyle PA, Cohen RA, Paul R, et al. Cognitive and motor impairments predict functional declines in patients with vascular dementia. Int J Geriatr Psychiatry, 2002. 17(2):164-9.

202. Chui H, Gonthier R. Natural history of vascular dementia. Alzheimer Dis Assoc Disord, 1999. 13 Suppl 3:S124-30.

203. Desmond DW, Erkinjuntti T, Sano M, et al. The cognitive syndrome of vascular dementia: implications for clinical trials. Alzheimer Dis Assoc Disord, 1999. 13 Suppl 3:S21-9.

204. Erkinjuntti T. Cognitive decline and treatment options for patients with vascular dementia. Acta Neurol Scand Suppl, 2002. 178:15-8. 
205. McPherson SE, Cummings JL. Neuropsychological aspects of vascular dementia. Brain Cogn, 1996. 31(2):269-82.

206. Roman GC. Stroke, cognitive decline and vascular dementia: the silent epidemic of the $21^{\text {st }}$ century. Neuroepidemiology, 2003. 22(3):161-4.

207. Meyer JS, Xu G, Thornby J, et al. Is mild cognitive impairment prodromal for vascular dementia like Alzheimer's disease? Stroke, 2002. 33(8): 1981-5.

208. van Swieten JC, Staal S, Kappelle LJ, et al. Are white matter lesions directly associated with cognitive impairment in patients with lacunar infarcts? J Neurol, 1996. 243(2):196-200.

209. Breteler MM, van Swieten JC, Bots ML, et al. Cerebral white matter lesions, vascular risk factors, and cognitive function in a populationbased study: the Rotterdam Study. Neurology, 1994. 44(7):1246-52.

210. Wolfe N, Babikian VL, Linn RT, et al. Are multiple cerebral infarcts synergistic? Arch Neurol, 1994. 51(2):211-5.

211. van Zagten M. Cerebral small-vessel disease. Vascular risk fatcor profiles, clinical manifestations, and disease progression in stroke. Doctoral Thesis. University of Maastricht, 1997.

212. Robertson IH, Murre JM. Rehabilitation of brain damage: brain plasticity and principles of guided recovery. Psychol Bull, 1999. 125(5):544-75.

213. Andersen G, Vestergaard K, Riis JO, Ingeman-Nielsen M. Dementia of depression or depression of dementia in stroke? Acta Psychiatr Scand, 1996. 94(4):272-8.

214. Desmond DW, Bagiella E, Moroney JT, Stern Y. The effect of patient attrition on estimates of the frequency of dementia following stroke. Arch Neurol, 1998. 55(3): 390-4.

215. Corea F, Henon H, Pasquier F, Leys D. Silent infarcts in stroke patients: patient characteristics and effect on 2-year outcome. J Neurol, 2001. 248(4):271-8.

216. Tatemichi TK, Paik M, Bagiella E, et al. Risk of dementia after stroke in a hospitalized cohort: results of a longitudinal study. Neurology, 1994. 44(10):1885-91.

217. Meyer JS, Obara K, Takashima S, et al. Problems encountered with longitudinal neurological, psychometric and cerebral CT imaging among stroke data bank patients with dementia. Neuroepidemiology, 1994. 13(6):340-4.

218. Pohjasvaara T, Mantyla R, Aronen HJ. Clinical and radiological determinants of prestroke cognitive decline in a stroke cohort. J Neurol Neurosurg Psychiatry, 1999. 67(6):742-8. 
219. Kempen GIJM, Brilman EI,Ormel J. De mini-mental state examination; normeringsgegevens en een vergelijking van een 12- en 20 item versie in een steekproef ouderen uit de bevolking. tijdschrift voor gerontologie en geriatrie, 1995. 26:163-172.

220. Rockwood K, Vascular cognitive impairment and vascular dementia. J Neurol Sci, 2002. 203-204(C):23-7.

221. Tzourio C, Anderson C. Blood pressure reduction and risk of dementia in patients with stroke: rationale of the dementia assessment in PROGRESS (Perindopril Protection Against Recurrent Stroke Study). PROGRESS Management Committee. J Hypertens Suppl, 2000. 18(1): S21-4.

222. Tzourio C, Anderson C, Chapman N, et al. Effects of blood pressure lowering with perindopril and indapamide therapy on dementia and cognitive decline in patients with cerebrovascular disease. Arch Intern Med, 2003. 163(9):1069-75

223. Chapman NR, Huxley, Anderson C, et al. Effects of a perindopril-based blood pressure lowering regimen on the risk of recurrent stroke according to stroke subtype and medical history: the PROGRESS Trial. Stroke, 2004. 35(1):116-21.

224. Aben I. Post Stroke Depression. The need for a bio-psycho-social approach. Doctoral Thesis University Maastricht, 2004.

225. Dubois B, Albert ML. Amnestic MCI or prodromal Alzheimer's disease? Lancet Neurol, $\backslash 2004.3(4): 246-8$. 
Publications 


\section{Articles}

Rasquin, SMC. (2002). Meer zicht op restverschijnselen. De Psycholoog, 37, 2, 75-76.

Rasquin SMC, Verhey FRJ, Lodder J. Vascular Cognitive impairment: een nieuw concept. Nederlands Tijdschrift voor Neurologie, 2003. 104 (4):209-214.

Rasquin SMC, Verhey FRJ, Lousberg R, Winkens I, Lodder J. Vascular cognitive disorders. Memory, mental speed and cognitive flexibility after stroke. Journal of the Neurological Sciences, 2002. 203-204:115-119.

Rasquin SMC, Lodder J, Ponds RWHM, Winkens I, Jolles J, Verhey FRJ. Cognitive functioning after stroke: a one-year follow-up study. Dementia and Geriatric Cognitive Disorders, 2004. 18:138-144.

Rasquin SMC, Verhey FRJ, Lodder J. The effect of different diagnostic criteria on the prevalence and incidence of post-stroke dementia. Neuroepidemiology. In press.

Rasquin SMC, Verhey FRJ, Lodder J. Predictive accuracy of MCI-subtypes for Alzheimer's disease and vascular dementia in subjects with mild cognitive impairment: a 2-year follow-up study. Dementia and Geriatric Cognitive Disorders. In press.

Rasquin SMC, Lodder J, Verhey FRJ. Predictors of reversible Mild Cognitive Impairment after stroke: a 2-year follow-up study. Journal of the Neurological Sciences. In press.

Rasquin SMC, Verhey FRJ, Oostenbrugge van RJ, Lousberg R, Lodder J. Demographic and CT-scan features related to cognitive impairment in the first year after stroke. Journal of Neurology, Neurosurgery, and Psychiatry. Journal of Neurology, Neurosurgery, and Psychiatry, 2004. 75:1562-1567

Rasquin SMC, Verhey FRJ, Lousberg R, Lodder J. Progressive brain damage in relation to cognitive performance after first-ever stroke: a 2-year follow-up study. Journal of Neurology, Neurosurgery, and Psychiatry. Revised version submitted. 
Heugten van CM, Rasquin SMC, Winkens I, Beusmans G, Verhey FRJ. Checklist for cognitive and emotional consequences following stroke (CLCE-24): development and initial validation. Submitted.

Rasquin SMC, Lodder J, Verhey FRJ. The influence of psychiatric symptoms on cognitive performance after stroke. Cerebrovascular Diseases. Revised version submitted.

Rasquin SMC, Heugten van CM, Winkens I, Beusmans G, Verhey FRJ. CheckLijst voor het opsporen van cognitieve en emotionele gevolgen na een beroerte (CLCE-24). Nederlands Tijdschrift voor Geneeskunde. Herziene versie ingediend.

\section{Abstracts}

Rasquin SMC, Verhey FRJ, Lousberg R, Winkens I \& Lodder J. CAMCOG: useful for early differentiation between Alzheimer and Vascular Dementia? International Psychogeriatrics, 2001, 13, 131s.

Rasquin, SMC, Verhey FRJ, Lousberg R, Lodder J. Second Congress on Vascular Dementia. Januari 2002, Salzburg. Vascular Cognitive Disorders.

Rasquin SMC, Verhey FRJ, Lousberg R, Winkens I \& Lodder J. Sixth EndoNeuro Meeting (Neurofederation). June 7 2002, Doorwerth. Influence of preexisting ischemic lesions on cognitive functioning in stroke patients.

Rasquin, SMC, Verhey FRJ, Lousberg R, Lodder J. Third Congress on Vascular Dementia. Oktober 2003, Praag. Vascular Mild Cognitive Impairment versus Mild Cognitive Impairment: is there a different course?

Rasquin, SMC, Lodder J, Verhey FRJ. Third Congress on Vascular Dementia. Oktober 2003, Praag. CT scan correlates of cognitive disorders after first ischemic stroke; a longitudinal study. 
Dankwoord 


\section{Lieve mensen,}

'Live as if you were to die tomorrow, learn as if you were to live forever' (Mahatma Gandhi).

Toen ik zo'n 5 jaar geleden begon als AIO wilde ik naast een interessante baan vooral ook mezelf verder ontwikkelen en elke dag weer nieuwe dingen leren. Achteraf bekeken zijn deze wensen dubbel en dik uitgekomen. Maar dat is niet vanzelf gegaan, daar heb ik veel hulp bij gehad. Iedereen bedanken is jammer genoeg niet mogelijk. Toch ga ik en poging wagen om de mensen te bedanken die mij op mijn ontdekkingsreis in de wetenschap een stapje verder hebben geholpen.

Als eerste wil ik de patiënten bedanken die vrijwillig aan het onderzoek hebben deelgenomen. Niet alleen door het verzamelen van de testgegevens, maar vooral door jullie verhalen heb ik geleerd wat een ingrijpende gevolgen een hersenberoerte kan hebben. De wetenschap is door jullie deelname weer een stapje vooruit gegaan.

Mijn twee promotoren en medebegeleider, professor Frans Verhey, professor Jaap Troost en Jan Lodder jullie hebben mij de meeste ontwikkelmogelijkheden gegeven en dat verdient een heel dikke Merci.

Beste Frans, ik heb vaak tegen collega's en vrienden gezegd dat ik heb geboft met mijn promotor en dat is ook zo. Ik heb bewondering voor de manier waarop jij telkens weer de teksten zo grondig en vooral ook kritisch nakeek. Jouw conceptuele visie op het onderzoeksproject heeft ervoor gezorgd dat ik alle zaken in het juiste perspectief zag.

Beste Jaap, $\mathrm{u}$ bent in een later stadium in het CODAS onderzoek gestapt. Toch bent $\mathrm{u}$ door $\mathrm{uw}$ interesse vanaf het begin betrokken geweest. Ik wil $\mathrm{u}$ danken voor de manier waarop ik op de afdeling neurologie heb kunnen werken, als psycholoog heb ik me thuis gevoeld tussen de artsen.

Beste Jan, wat kan een AIO zich nog meer wensen dan een begeleider zoals jij? Jouw motivatievermogen is onbegrensd. Ik heb genoten van onze discussies, met jou als advocaat van de duivel. Elke keer als ik bij zo'n gesprek vandaan kwam begon ik weer met plezier hout te sprokkelen. Ik durf, dankzij jou 3 december het ravijn blindelings over te steken! 
Wat zou een AIO zijn zonder gedegen statistiek begeleiding? Beste Richel Lousberg, met veel geduld en precisie heb je me wegwijs gemaakt in de wirwar van statistische analyses. Door jouw hulp bij de analyses zijn de resultaten voor mij gaan leven en werden de getallen een verhaal.

Een onderzoek zoals dit kun je niet in je eentje uitvoeren. Ik heb dan ook ondersteuning gehad van geweldige onderzoeksmedewerkers, Ieke Winkens, Esmeralda van Pol en Birgit Senden. Jullie hebben bergen werk verzet en dat is een ontzettend lekker gevoel! Ieke, hoe je het gedaan hebt om van 2 onderzoeken van elke patiënt het ID-nummer te weten en ook nog eens het verhaal achter de patiënt ik weet het niet, maar ik weet wel dat ik altijd heel blij met je ben geweest. Ook de stagiaires Chantal Geusgens, Tessa Versteegde en Yvonne Leppers, hebben een steentje bijgedragen aan het onderzoek. Door jullie te mogen begeleiden heb ik veel geleerd. Vooral Chantal, je was eigenlijk ook onderzoeksmedewerker. Toen jij bij een van de eerste besprekingen met het overzicht van je statistische analyses kwam dacht ik: 'Ze zal wel gelijk hebben, maar echt begrijpen doe ik het niet'. Nu ben je zelf AIO, net als Ieke en dat vind ik erg leuk, vooral omdat we nu weer collega's van elkaar zijn.

Artikels worden alleen maar beter als experts mee willen publiceren. Caroline van Heugten, Robert-Jan van Oostenbrugge, Rudolf Ponds, Pieter-Jelle Visser en professor Jelle Jolles jullie expertise was een belangrijke bijdrage aan dit proefschrift. Beste Caroline, om middels het CODAS onderzoek te mogen meehelpen aan jouw onderzoek was een leerzame ervaring. Jij vertaalde mijn resultaten in een vragenlijst, op deze manier werd het onderzoek en stuk levendiger en kreeg ik goede gesprekken met de patiënten. Ik vind het een eer dat ik in jouw programma Cognitieve Revalidatie mag werken en ik hoop (en weet eigenlijk wel zeker) dat ik nog veel van je zal leren! Beste Robert, wat een klus heb jij geklaard: 200 CT scans een voor een beoordelen. Meer nog dank voor je kritische blik op ons artikel en later op onze aanvraag, nu maar hopen dat die wordt gehonoreerd. Rudolf, jij hebt, samen met Frans, alle patiëntendossiers nagekeken om de diagnose dementie te kunnen stellen. Geen enkel detail ontging je, waardoor ik met stelligheid in mijn artikels kan zeggen jij een van de 2 experts was die de diagnose stelde. Beste Pieter-Jelle dank voor het mogen 'lenen' van het MCI-bestand en voor je gedegen input aan het artikel. Beste Jelle, jij voelde net als ik aan dat er toch iets moest gebeuren met de missing data. Dat jouw manier van implementatie naadloos overeenkomt met de statistische analyse is een bewijs voor je goede klinische blik. Beste Jane Sykes, ik dank u voor het nakijken van mijn Engels.

De collega's van de afdeling neurologie ben ik veel dank verschuldigd. Beste Anita Boreas, Manuela Voorend, Rob Rouhl, Lisette van Raak en de mensen 
van het secretariaat, jullie waren het neurologisch brein achter het verzamelen van de patiëntgegevens. Die klus had ik niet kunnen klaren, daar had ik eerst geneeskunde voor moeten gaan studeren.

Lieve collega's wat was het fijn om met jullie samen te werken. De mede AIO's en met name Ivo Aben wil ik vooral bedanken voor de gezellige AIObijeenkomsten, het is wonderbaarlijk wat je allemaal van elkaar kunt leren! Beste Pauline Aalten, als kamergenootje heb je zo'n beetje de hele AIO-periode met mij doorgemaakt. Vooral de congressen naar Nice en Canterburry waren de krenten in de pap. Susan van Hooren, lieve Susan, je bent niet alleen een collega, maar vooral ook een goede vriendin. De gesprekken die wij samen hebben gevoerd waren gezellig en als het nodig was ook een steun in de rug. Ik vind het erg leuk dat ik straks jouw paranimf mag zijn en ik weet zeker: dat wordt een toppromotie! Susanne Valentijn, Petra Hurks en Jeroen Schmitt, lieve Suus, Petra en Jeroen, jullie gezelligheid kent geen grenzen. Altijd waren jullie wel in voor een borrel of een etentje. Gelukkig houdt dit niet op met het einde van ons AIO-bestaan!

Beste Ankie Hochstenbach, Laurent Louwies, Marco van Hertrooy, Nico Rozendaal en Jacqueline Wetzelaer jullie hadden altijd tijd om even dat kleine vraagje van mij op te lossen en te zorgen dat ik als vanzelf weer verder kon.

Beste Kitty Verwoerdt, dank voor je ondersteuning bij het verspreiden van de boekjes, maar vooral dank dat je elke week weer een plekje in de agenda van Frans voor mij wist vrij te plannen (ik weet dat dat niet gemakkelijk is!). Geert Leenders jou enthousiasme houdt nooit op en dat is maar goed ook. Mijn 'nieuwe' collega's van het iRv wil ik bedanken voor de interesse tijdens de afronding van mijn proefschrift.

Lieve vrienden, jullie waren altijd heel geïnteresseerd in wat ik deed, dat heb ik erg gewaardeerd. De uurtjes kletsen, de etentjes en de stapavondjes maken voor mij het leven zeer aangenaam en wat mij betreft: laten we maar weer iets afspreken!

Lieve Sjuf en Ria jullie gastvrijheid en warmte zorgen ervoor dat ik me thuis voel.

Beste mensen van de leescommissie, dank dat jullie mijn proefschrift hebben willen beoordelen. Jullie reacties waren erg spontaan en positief, dat beloofd wat voor 3 december!

Langzaam aan komt dan toch de grote dag in zicht. Ik verheug mij er erg op en $\mathrm{ik}$ ben blij dat ik dit mooie moment mag delen met mijn twee lieve paranimfen. Lieve Joyce, mijn kleine zusje, maar in zoveel opzichte groter dan ik, ik ben blij 
dat wij al ons hele leven zoveel plezier samen hebben. Ik ben er erg trost op dat jij straks mijn paranimf wilt zijn. Lieve Marjolein, we zijn ooit begonnen als verre studiegenootjes toen werden we collega's en nu zijn we vrienden. Jij hebt met mij lief en leed (lees: veel lief en gelukkig weinig leed) gedeeld de laatste 5 jaar en dat voelt ontzettend goed. Ik vind het een eer dat ik jouw paranimf heb mogen zijn en ik ben blij dat jij dat straks ook bij mij bent.

Lieve papa en mama, dankzij jullie ben ik geworden wie ik ben. Ik wil jullie heel erg bedanken voor jullie onvoorwaardelijke steun bij alles wat ik heb gedaan. Jullie hebben me de mogelijkheid en vooral ook de vrijheid gegeven te doen en te laten wat ik graag wil en dat maakt dat geluk voor mij heel gewoon is! Dit boekje is dan ook voor jullie.

Lieve Xavier, ik heb dit AIO-avontuur kunnen doen omdat wij het samen zo goed hebben. Wat is het fijn te weten dat er altijd iemand voor je is, die steeds weer voor je klaarstaat. Met jou wil ik dan ook vele nieuwe ontwikkeling meemaken!

Liefs Sascha 
Curriculum Vitae 
Sascha Rasquin werd geboren op 20 november 1976 te Maastricht. In 1995 deed ze eindexamen Moderne Talen-Wetenschappen aan de Provinciale Secundaire School Voeren (B). Hierna ging ze psychologie studeren aan de Universiteit Maastricht. In 1999 werd het doctroaal diploma Psychologie behaald, met als hoofdrichting biologische psychologie. In september 1999 begon ze aan een dienstverband als Assistent in Opleiding bij de afdelingen Psychiatrie \& Neuropsychologie en Neurologie aan de Universiteit Maastricht, om het onderzoek uit te voeren dat in dit proefschrift is beschreven. Sinds mei 2004 is zij werkzaam als projectleider binnen het programma Cognitieve Revalidatie van het iRv te Hoensbroek. 
